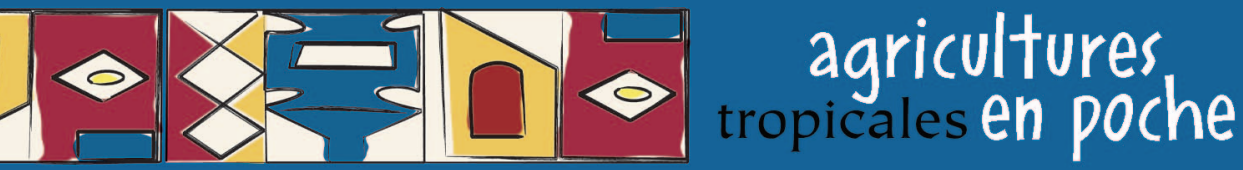

Appuyer

les organisations

de producteurs

M.J. Dugué, D. Pesche, J.F. Le Coq

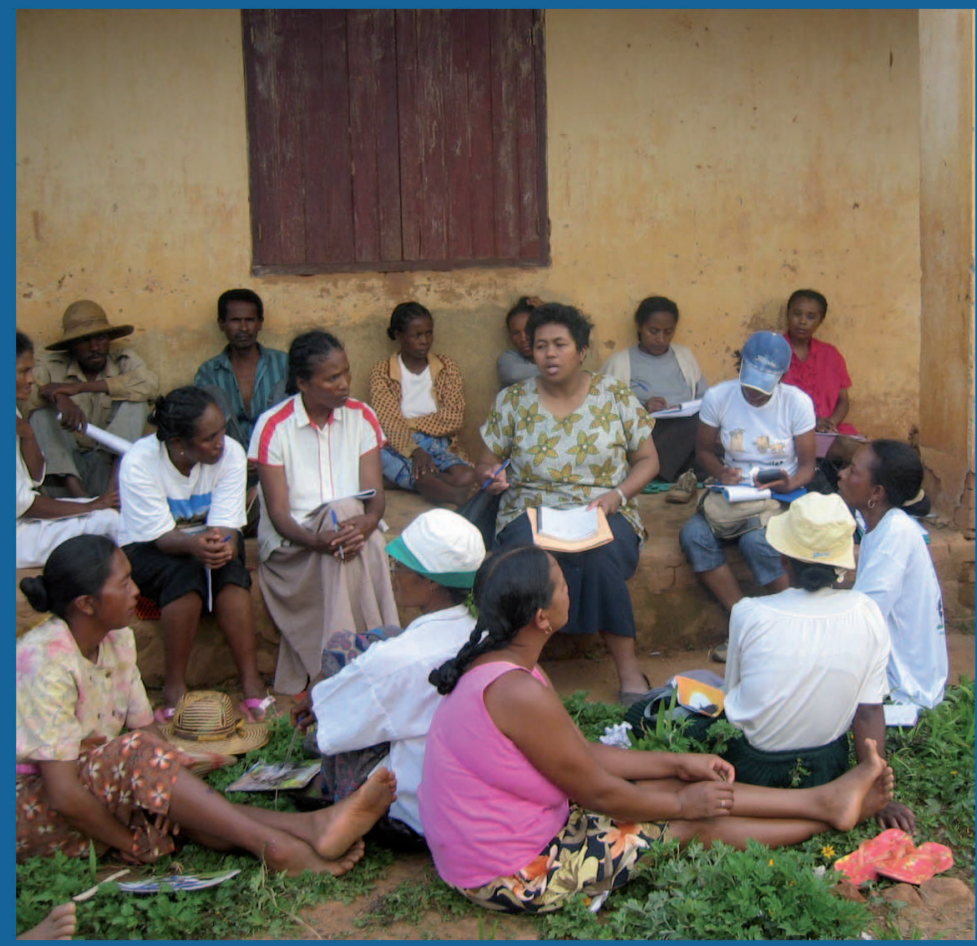

Quæ Cta

Presses agronomiques de Gembloux 

Agricultures tropicales en poche

Directeur de la collection

Philippe Lhoste

\section{Appuyer les organisations de producteurs}

Marie-Jo Dugué, Denis Pesche, Jean-François Le Coq

Éditions Quæ, CTA, Presses agronomiques de Gembloux 
Le Centre technique de coopération agricole et rurale (CTA) est une institution internationale conjointe des États du Groupe ACP (Afrique, Caraïbes, Pacifique) et de l'Union européenne (UE). Il intervient dans les pays ACP pour améliorer la sécurité alimentaire et nutritionnelle, accroître la prospérité dans les zones rurales et garantir une bonne gestion des ressources naturelles. Il facilite l'accès à l'information et aux connaissances, favorise l'élaboration des politiques agricoles dans la concertation et renforce les capacités des institutions et communautés concernées.

Le CTA opère dans le cadre de l'Accord de Cotonou et est financé par l'UE.

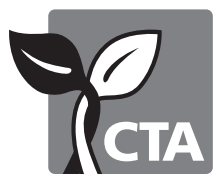

CTA, Postbus 380, 6700 AJ Wageningen, Pays-Bas

www.cta.int

Éditions Quæ, RD 10, 78026 Versailles Cedex, France

www.quae.com

Presses agronomiques de Gembloux, Passage des Déportés, 2, B-5030 Gembloux, Belgique

www.pressesagro.be

(C) Quæ, CTA, Presses agronomiques de Gembloux 2012

ISBN (Quæ) : 978-2-7592-1806-6

ISBN (CTA) : 978-92-9081-501-3

ISBN (PAG) : 978-2-87016-121-0

ISSN : $1778-6568$

(C) Le code de la propriété intellectuelle du $1^{\text {er }}$ juillet 1992 interdit la photocopie à usage collectif sans autorisation des ayants droit. Le non-respect de cette disposition met en danger l'édition, notamment scientifique. Toute reproduction, partielle ou totale, du présent ouvrage est interdite sans autorisation des éditeurs ou du Centre français d'exploitation du droit de copie (CFC), 20, rue des Grands-Augustins, 75006 Paris. 


\section{Table des matières}

Avant-propos......................................... 5

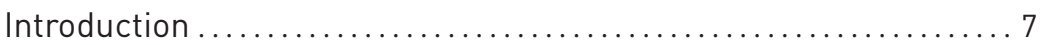

\section{Partie 1}

Pourquoi appuyer les organisations de producteurs?

1. D'où viennent les organisations de producteurs? ............ 15

Le contexte global et les grandes étapes de l'expansion des OP ........ 15

La diversité des formes d'organisation des producteurs............. 17

Pour aller plus loin ..................................... 23

2. Quels rôles jouent les organisations de producteurs ?........ 25

Des résultats réels mais parfois difficiles à apprécier ................ 25

Les $\mathrm{OP}$ face à des défis nécessitant des capacités renforcées........... 28

Des arguments en faveur du renforcement des OP ................ 32

\section{Partie 2 \\ Renforcer les organisations de producteurs de l'intérieur}

3. Établir le cadre de l'organisation de producteurs. ............ 39

La structure d'une organisation ............................. 39

Lidentité et les systèmes de valeurs.......................... 44

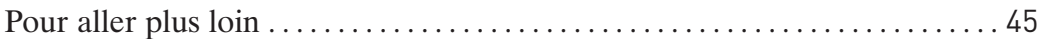

4. Définir les orientations d'une organisation de producteurs

et les respecter .................................... 47

La planification et l'élaboration d'un plan stratégique $\ldots \ldots \ldots \ldots \ldots \ldots 47$

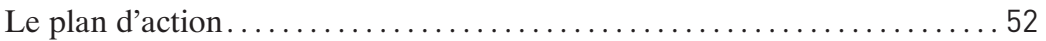

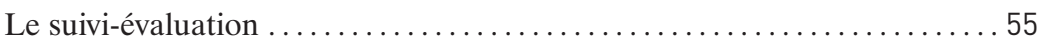

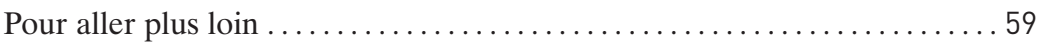

5. Faire fonctionner une organisation de producteurs .........61

Le cadre : les textes qui régissent l'OP ......................61

La prise de décision et l'exercice du pouvoir $\ldots \ldots \ldots \ldots \ldots \ldots \ldots \ldots 62$

La gestion des ressources financières .......................... 64

La gestion des ressources humaines ........................6 67

La gestion de l'information et la communication .................. 71

Légitimité et représentativité :

les enjeux du fonctionnement pour les organisations ............... 74 
6. Renforcer les capacités des organisations de producteurs . . 77

Le renforcement de capacités ou la formation .................... 77

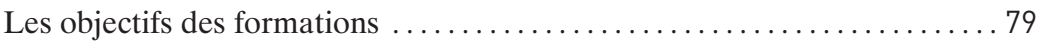

Lélaboration d'un plan de formation $\ldots \ldots \ldots \ldots \ldots \ldots \ldots \ldots \ldots \ldots \ldots$

Quels moyens pour la formation? ........................ 83

Le cas particulier de la formation initiale au métier d'agriculteur ....... 85

Limpact du renforcement des capacités. ....................... 86

Pour aller plus loin ................................... 86

\section{Partie 3 \\ Renforcer les organisations de producteurs dans leurs relations avec leur environnement}

7. Les partenariats à visée technique

Laccès des producteurs aux marchés : le rôle des OP dans la coordination et la gestion des filières .................................. 90

Les OP et le financement de l'agriculture ...................... 94

Les OP et l'innovation technique ........................... 97

Les OP et la gestion de biens partagés........................ 99

Pourquoi des partenariats? Quels partenariats? ................. 100

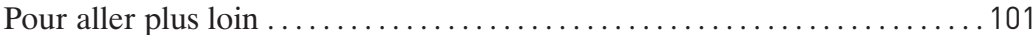

8. La participation des organisations de producteurs à la définition des politiques publiques ................... 103

De nombreuses OP sont concernées par la définition des politiques..... 103 Les $\mathrm{OP}$ cherchent à influencer les politiques dans un contexte

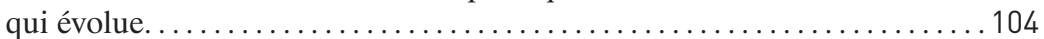

Linfluence politique se joue d'abord au niveau national ............ 106 Lintervention des OP est nécessaire à l'échelle supranationale.......... 109 Constats, interrogations et pistes d'avenir..................... 110 Pour aller plus loin .................................... 113

9. Le diagnostic des organisations de producteurs........... 115

Les objectifs du diagnostic ................................ 115

Quelques principes généraux............................. 116

Des outils de diagnostic................................ 118

Conclusion - Pour des programmes complets et cohérents d'appui aux organisations de producteurs ................. 125

Quelles OP veut-on appuyer et autour de quelles grandes fonctions? ... 126 Quelles actions mettre en œuvre? Quels moyens mobiliser et par qui? . . 131 Perspectives........................................... 136

Bibliographie......................................... 139

Liste des sigles ...................................... 141

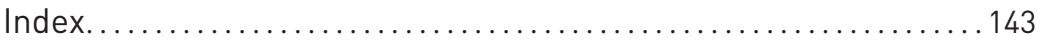

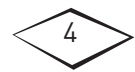




\section{Avant-propos}

La collection «Agricultures tropicales en Poche»a été créée par un consortium comprenant le CTA de Wageningen (Pays-Bas), les Presses agronomiques de Gembloux (Belgique) et les éditions Quæ (France). Cette nouvelle collection, comme l'était celle qui l'a précédée («le Technicien d'Agriculture tropicale» chez Maisonneuve et Larose), est liée à la collection anglaise, "The Tropical Agriculturist», chez Macmillan (Royaume-Uni). Elle comprend trois séries d'ouvrages pratiques consacrés aux productions animales, aux productions végétales et aux questions transversales.

Ces guides pratiques sont destinés avant tout aux producteurs, aux techniciens et aux conseillers agricoles. Ils se révèlent être également d'utiles sources de références pour les chercheurs, les cadres des services techniques, les étudiants de l'enseignement supérieur et les agents des programmes de développement rural.

Le présent ouvrage était attendu pour enrichir la série consacrée aux thèmes transversaux, sur un sujet d'actualité : il est consacré à l'appui aux organisations de producteurs (OP). Celles-ci, de nature très diverse, connaissent une forte dynamique dans les pays en développement et sont l'objet de nombreux travaux et de rencontres de professionnels et de spécialistes de ce sujet.

Les trois auteurs de ce manuel ont effectué un travail collectif coordonné par Marie-Jo Dugué. Ils ont fait le choix d'un ouvrage synthétique qui fournit au lecteur un cadre de réflexion lui permettant de se poser les bonnes questions et de s'orienter, le cas échéant, vers d'autres sources qui fournissent des outils plus approfondis. La construction de l'ouvrage est très didactique : des encadrés définissent, au fil de la lecture, les principaux concepts utiles; d'autres encadrés présentent des études de cas qui illustrent la démarche proposée; des schémas synthétisent les démarches utiles, et des pistes (documents et sites Internet) sont proposées au lecteur «pour aller plus loin».

Les auteurs de ce manuel se sont largement inspirés de l'expérience du module de formation «Organisation des producteurs» proposé jusqu'en 2011 par l'Institut des régions chaudes (IRC) de Montpellier dans le cadre du mastère «Acteurs du développement rural», module dont ils ont été animateurs depuis 2004, à la suite de Marie-Rose Mercoiret et Jacques Berthomé, qui avaient élaboré en 1992 ce module 
du mastère «Vulgarisation et organisations professionnelles agricoles ». Ces enseignements ont été conçus pour répondre aux besoins repérés dans les diverses expériences d'accompagnement des OP, au cours des vingt dernières années par les équipes du CIRAD et du CIEPAC. Cet ouvrage, qui en est le prolongement, propose une synthèse actualisée de ces éléments de formation et d'aide à la décision.

Les auteurs de cet ouvrage ont tous trois participé à ce travail au service des OP, qui a été fondé à la fois sur des actions de terrain auprès d'organisations de divers niveaux, sur un partenariat avec des opérateurs de développement accompagnant les OP et sur de multiples actions de formation à destination de responsables et de membres d'organisations aussi bien que de structures d'appui.

Ces auteurs présentent des profils complémentaires : la coordinatrice de la rédaction, Marie-Jo Dugué, a travaillé au renforcement des capacités des OP et à des actions de formation; Denis Pesche et Jean-François Le Coq, chercheurs au CIRAD, sont engagés dans des démarches de recherche-action sur différents terrains de coopération avec les pays du Sud.

Cet ouvrage constitue donc un outil de travail synthétique et complet pour permettre aux professionnels et acteurs des organisations de producteurs d'y voir plus clair dans leurs pratiques et d'améliorer leurs démarches dans différents domaines : appuis, diagnostics, suiviévaluation, planification, gestion, formation, etc. Il constitue un excellent guide dans un domaine complexe permettant d'accéder, le cas échéant, à des outils plus spécialisés.

Philippe Lhoste, Directeur de la collection Agricultures tropicales en Poche 


\section{$\ll$ Introduction}

Les organisations de producteurs (OP) dont nous allons parler dans cet ouvrage sont des formes d'organisations assez récentes dans les pays en développement. Nées il y a quelques décennies pour les plus anciennes, elles sont aujourd'hui nombreuses dans la plupart des pays. Après avoir caractérisé, à grands traits, leur nature nous présentons dans ce chapitre introductif le plan de l'ouvrage.

Les OP sont nombreuses et diverses; l'importance des fonctions qu'elles remplissent est largement reconnue par les acteurs du développement, ainsi que l'impossibilité de voir ces rôles assumés légitimement par d'autres acteurs. Or, il apparaît que les OP ne disposent pas toujours des capacités et des moyens pour remplir ces rôles avec toute l'efficacité souhaitable. C'est pourquoi au cours des dernières années, de nombreux partenaires du développement ont investi dans des actions visant à renforcer leurs capacités et dans la production de documents pédagogiques ou méthodologiques. Ces ouvrages adoptent des formats et des portes d'entrée diverses en réponse aux objectifs spécifiques de leurs initiateurs.

Ce manuel ne prétend en aucun cas remplacer ces outils. De par son format, il ne saurait constituer un support méthodologique approfondi sur l'ensemble des thèmes abordés, ni un recueil de recettes pour créer ou appuyer des OP. Il répond plutôt au souci de proposer un cadre de réflexion général et cohérent aidant ses lecteurs à «se poser les bonnes questions» afin de tirer parti des multiples autres ressources documentaires existantes : il s'agit donc de fournir quelques clés pour comprendre, pour agir plus efficacement.

Il s'adresse à des personnes impliquées dans le renforcement des capacités des OP. Ce peut être des consultants, des agents de projets, des fonctionnaires en charge d'un service d'appui aux OP, mais aussi des élus ou des salariés engagés au sein même de ces organisations.

Les organisations auxquelles il est fait référence sont pour la plupart de taille moyenne et regroupent des agriculteurs familiaux, ce qui ne signifie pas que les principes énoncés ne sont pas pertinents également pour d'autres catégories d'OP.

Ces choix résultent de l'expérience d'accompagnement des OP acquise depuis plus de vingt ans par les équipes du CIRAD (Centre de coopération internationale en recherche agronomique pour le développement) 
et du CIEPAC (Centre international pour l'éducation permanente et l'action concertée). Les auteurs de cet ouvrage ont participé à ce travail au service des OP, qui était fondé à la fois sur des actions de terrain auprès d'organisations de divers niveaux, sur un partenariat avec des opérateurs de développement accompagnant les OP et sur de multiples actions de formation à destination de responsables, de membres d'organisations et d'acteurs d'appui. Ces activités ont donné lieu à de nombreux rapports dont certains ont été publiés et sont cités dans ce manuel. Parmi les actions de formation, les auteurs ont notamment animé ces dernières années le module «Organisation des producteurs» du mastère «Acteur du développement rural» (ex «Vulgarisation et organisations professionnelles agricoles»), proposé jusqu'en 2011 par l'Institut des régions chaudes (IRC) à Montpellier. Le contenu des parties 2 et 3 de ce manuel est assez largement inspiré des enseignements dispensés dans ce cadre. Ces enseignements ont puisé leur inspiration dans de nombreuses situations professionnelles où les auteurs ont été amenés à participer à l'accompagnement d'OP ou à débattre des enjeux auxquels elles font face et des appuis qu'elles reçoivent.

On trouvera ci-après des éléments d'analyse, des principes et des repères méthodologiques plutôt que des outils clés en main. Au fil du texte, quelques encadrés «études de cas» illustrent des points particuliers. Des encadrés d'approfondissement visent par ailleurs à définir et expliciter les nombreux concepts et notions employés aujourd'hui dans le monde du développement rural, et à décrire brièvement les démarches les plus courantes, afin de permettre au lecteur de s'orienter dans un paysage très riche mais parfois confus.

À la fin de chaque chapitre sont indiquées les références de documents plus spécialisés parus récemment, pour la plupart téléchargeables en accès libre sur Internet, ces références ne prétendent pas être exhaustives. Cela permettra aux lecteurs et lectrices d'aller plus loin s'ils le souhaitent. Une bibliographie générale d'ouvrage complètera la documentation accessible sur ce sujet.

\section{Les «OP»: de quoi parle-t-on? Quelques définitions}

Les organisations paysannes, ou de producteurs (OP), sont entendues ici comme des organisations d'agriculteurs familiaux, d'éleveurs, de pêcheurs artisanaux, de paysans sans terre ou d'indigènes qui sont

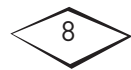


structurées, au-delà des communautés de base, à des échelles locale, nationale ou régionale. Elles revêtent des formes très variables selon les localisations et les périodes tant en ce qui concerne leur taille, les niveaux auxquels elles se structurent, les enjeux autour desquels elles se construisent, que les objectifs qu'elles se fixent et les modes d'action qu'elles adoptent.

\section{Questions de terminologie}

Labréviation «OP» peut recouvrir différentes significations, évoquant des visions particulières du rôle de ces organisations mais aussi du modèle d'agriculture auquel on se réfère. Le terme "organisation de producteurs» souvent considéré comme le plus neutre, met toutefois l'accent sur la fonction de production. C'est pourquoi on utilise également «organisation de producteurs ruraux» (OPR) pour souligner leur insertion dans le monde rural, souvent source d'activités et de revenus plus diversifiés que la seule production agricole. Le terme «organisation paysanne» fait référence à la notion de paysan, de pays et souligne souvent la dimension de défense des intérêts des petits agriculteurs familiaux; les expressions «organisation de producteurs» ou «organisation paysanne» sont les traductions les plus fidèles de «farmers' organisations» et «organizaciones campesinas».

Le terme «organisation professionnelle agricole», directement inspiré de l'histoire agricole française, fait référence au métier d'agriculteur, à leur profession et évoque souvent la fonction de représentation des organisations notamment dans un contexte de cogestion ou de partenariat avec l'État.

Pour plus de facilité, nous utiliserons généralement dans cet ouvrage l'abréviation OP, en précisant si nécessaire de quelle catégorie d'organisation il est question.

Les OP expriment une volonté, affirmée ou émergente, des ruraux de résoudre des problèmes par l'action collective, et de relever des défis auxquels ils sont confrontés. Dans leur diversité, elles présentent des caractéristiques communes. Le tableau 1 résume les principaux critères qui permettent de distinguer ces catégories d'organisations qui coexistent en milieu rural.

- Elles sont fondées sur la libre adhésion. Leur fonctionnement se réclame de principes démocratiques et elles entretiennent des relations avec d'autres acteurs qui agissent dans leur environnement.

- Structures d'interface entre les sociétés locales et leur milieu, elles ont pour vocation d'améliorer les relations de leurs membres avec leur 
environnement économique, institutionnel ou politique et de le rendre plus favorable à leurs initiatives.

- Les OP relèvent du secteur privé ainsi que le mettent en évidence les statuts qu'elles adoptent en fonction des réglementations en vigueur dans les différents pays. Ce sont des coopératives et groupements de producteurs; des groupements d'intérêt économique; des groupements d'intérêt rural; des unions, fédérations et confédérations regroupant un nombre variable d'organisations de base, locales ou régionales; des associations; etc. Tantôt spécialisées dans un produit ou une fonction, tantôt ayant un large éventail d'activités, elles produisent des biens privés destinés à leurs membres. Cette activité peut se traduire par l'approvisionnement groupé pour les intrants et les équipements productifs individuels, la mise en marché collective des productions des adhérents, l'acquisition et la gestion d'équipements collectifs pour la production (par exemple l'irrigation), le stockage ou la transformation des produits, etc.

Tableau 1. Critères de différenciation des formes d'organisations qui coexistent en milieu rural.

\section{Forme d'organisation Organisation de producteurs Organisation coutumière}

\begin{tabular}{|c|c|c|}
\hline Base d'adhésion & Libre et volontaire & $\begin{array}{l}\text { Automatique : déterminée } \\
\text { par l'appartenance sociale } \\
\text { (classe d'âge, etc.) }\end{array}$ \\
\hline
\end{tabular}

Aire d'intervention De locale à sous-régionale, Strictement locale voire internationale selon l'OP

\begin{tabular}{lll}
\hline Rôle premier & Interface entre & Régulation sociale à \\
& producteurs ruraux & l'échelon local entre groupes \\
et environnement & et individus
\end{tabular}

\begin{tabular}{lll}
\hline Statut & $\begin{array}{l}\text { Droit privé : association } \\
\text { ou coopérative }\end{array}$ & $\begin{array}{l}\text { Variable : souvent } \\
\text { pas de statut formel }\end{array}$ \\
\hline $\begin{array}{l}\text { Compétences et } \\
\text { objectifs définis par }\end{array}$ & Les membres : volontaires & La tradition \\
\hline Ressources & $\begin{array}{l}\text { Cotisations des membres, } \\
\text { produits d'activités propres, } \\
\text { ressources issues de partenariat }\end{array}$ & Dons des «membres» \\
\hline Représentants & Élus par les membres & $\begin{array}{l}\text { Charges héréditaires, } \\
\text { délégation de pouvoir } \\
\text { ou autre }\end{array}$ \\
\hline
\end{tabular}


- Dans la très grande majorité des cas cependant, les OP produisent aussi ou contribuent à la production de biens publics à travers la mise en place d'appuis aux producteurs (information, conseil agricole ou rural, formation), le soutien qu'elles apportent à l'innovation technique, économique et organisationnelle au sein des unités de production familiales et des sociétés locales, et leur implication parfois importante dans la gestion des ressources naturelles. Certaines OP interviennent aussi dans le secteur social et s'efforcent d'améliorer les conditions d'existence des ruraux. Elles tentent alors de pallier les défaillances des pouvoirs publics (État central, collectivités territoriales décentralisées) dans la fourniture de biens et de services collectifs d'intérêt général comme l'éducation et la santé.

- À des rythmes et selon des modalités variables, les OP développent des actions dans le champ du politique, aux échelons local, régional, national, sous-régional et de plus en plus souvent international. Elles s'efforcent de peser sur les différents centres de décisions publiques

$\begin{array}{lll}\begin{array}{l}\text { Organisation communautaire } \\ \text { de base }\end{array} & \begin{array}{l}\text { Collectivité territoriale } \\ \text { décentralisée }\end{array} & \text { ONG d'appui }\end{array}$

En général liée aux

organisations coutumières
Pas d'adhésion volontaire: Libre et volontaire

élection sur une base territoriale

Locale

Locale à régionale

De locale à sous-régionale, voire internationale selon l'ONG $\begin{array}{ll}\text { Production et gestion } & \text { Opérateur de } \\ \text { de biens publics : définition } & \text { développement }\end{array}$ et mise en œuvre des politiques locales de développement; administration du territoire

Droit public, régi par la loi : organe public décentralisé

La loi de décentralisation privé : association ou société de droit privé

Partenaires et membres de la communauté qui participent

Contribution des habitants de la communauté et partenariat

Impôts et taxes parafiscales

Membres : volontaires
Financement privé ou de la coopération internationale

Délégué : élection, nomination, Élus selon code électoral etc. par les habitants de la communauté

Élus par les membres 
et d'influer sur la définition des politiques publiques. Elles cherchent aussi à influer sur les acteurs privés dans les filières. Pour cela, elles s'engagent dans des processus ouverts, pérennes et institutionnalisés, de concertation et de négociation avec les pouvoirs publics et des acteurs privés. Cela permet aux ruraux de participer (1) à l'élaboration et à la mise en œuvre de stratégies de développement local (ou régional) en relation avec les collectivités territoriales décentralisées; (2) à la réorganisation de filières de produits; (3) à l'élaboration de politiques sectorielles (foncier, financement ou crédit, conseil agricole, etc.). Certaines OP se structurent en réseaux et cherchent ainsi à peser sur les orientations des politiques de développement agricole et rural à l'échelon national et régional (UEMOA, Mercosur) et sur les centres de décisions internationaux.

Ces caractéristiques communes à la très grande majorité des OP les distinguent d'autres formes d'organisations qui préexistaient dans les sociétés locales ou qui y ont été introduites plus ou moins récemment.

\section{Structure de l'ouvrage}

La première partie analyse pourquoi les partenaires ont besoin des OP en revenant sur les conditions de leur création (chapitre 1) puis en examinant les rôles qu'elles jouent (chapitre 2). Il en ressort que le renforcement des OP est nécessaire.

La deuxième partie traite des moyens de renforcer les OP en interne, dans les domaines qui sont de leur responsabilité exclusive, qu'il s'agisse de leur structure (chapitre 3), de la définition des orientations (chapitre 4), du fonctionnement (chapitre 5) ou du renforcement des capacités (chapitre 6).

Dans la troisième partie on s'intéresse au renforcement des OP dans les relations qu'elles établissent avec leur environnement, sur le plan technique (chapitre 7), ou quand elles s'impliquent dans la définition des politiques publiques (chapitre 8). La démarche de diagnostic des organisations contribue à faire des OP des partenaires plus «efficaces », cela fait l'objet du chapitre 9.

La diversité et la complémentarité des champs dans lesquels il est pertinent de renforcer les OP plaident en faveur d'actions coordonnées, c'est ce qui est analysé en conclusion. 


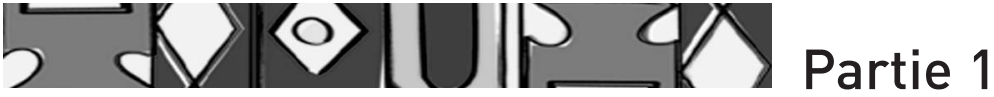

\section{Pourquoi appuyer les organisations de producteurs?}

Cette question générale peut paraître étrange. Pourtant, il n'est pas rare de constater qu'un représentant d'un ministère ou d'un bailleur de fonds estime que le renforcement des organisations de producteurs (OP) n'est pas une priorité. Renforcer les capacités des OP à gérer et mieux réaliser leurs activités, pourquoi pas? Mais renforcer les OP pour qu'elles deviennent un acteur plus présent dans les processus politiques et la définition des choix de projets ou de programmes pour le secteur rural n'est pas toujours une démarche facilement acceptée par des acteurs qui ont toujours eu le premier rôle dans ce domaine.

Avant d'aborder les principes et méthodes de l'appui aux OP, cette première partie vise donc à répondre à trois questions préalables : que sont les OP? À quoi servent-elles? Pourquoi ont-elles besoin d'appui?

Pour cela, après avoir décrit l'histoire et la diversité des organisations qui en a résulté (chapitre 1), on analysera les rôles joués par les OP, les résultats qu'elles ont obtenus et les défis auxquels elles sont confrontées, d'où il ressort que des organisations solides sont utiles voire nécessaires au développement rural (chapitre 2). Il ne s'agit pas d'en faire des organisations fétiches, qu'il faudrait renforcer à tout prix, mais plus simplement de prendre conscience que les processus de modernisation et de transition des agricultures ont toujours été accompagnés de la création et de l'essor de nombreuses OP qui y ont joué un rôle souvent central.

Ces deux chapitres sont fondés notamment sur les travaux préparatoires à un atelier qui a rassemblé des responsables d'OP de trois continents et plusieurs spécialistes reconnus de l'accompagnement des OP (Mercoiret et al., 2007). 



\section{$\sqrt{1 .}$ D'où viennent les organisations de producteurs?}

Pourquoi se poser cette question? Parce que comprendre d'où l'on vient explique à la fois la diversité des rôles et des fonctions joués actuellement par les OP, et les formes multiples d'organisation qui coexistent. On conçoit bien que pour appuyer les $\mathrm{OP}$ - perspective dans laquelle se situe cet ouvrage -, il est essentiel de prendre en compte cette hétérogénéité.

Créées à l'initiative d'opérateurs de développement (projets ou administrations), de petits groupes de producteurs ou d'autres organisations de la société civile, les OP sont aujourd'hui des organisations souvent complexes, à l'histoire riche. Elles sont de plus en plus reliées entre elles dans des fédérations, réseaux et mouvements.

La diversité actuelle s'est construite progressivement en réponse à l'évolution du contexte socio-économique et politique des 30 dernières années. Pour conforter la structuration des organisations de producteurs tout en respectant l'identité et la dynamique propre de leurs organisations, il est utile de comprendre ces interactions.

\section{Le contexte global et les grandes étapes de l'expansion des OP}

Au début des années 1980, il existait dans quelques pays des mouvements d'organisations plus anciens, souvent coopératifs, qui étaient très liés aux politiques d'intervention de l'État qui pouvait exercer un contrôle étroit sur ces organisations. Au Sahel, des organisations sont nées dans les années 1970 pour faire face aux sécheresses et aux conditions d'existence difficiles en milieu rural. Mais dans l'ensemble, c'est au tournant des années 1980 que naît la grande majorité des OP qui existent aujourd'hui et dont nous allons parler dans cet ouvrage.

Depuis cette prériode en effet, le contexte économique et institutionnel de l'agriculture a connu de profonds changements : désengagement de l'État de l'appui à l'agriculture et privatisation des fonctions 
économiques (approvisionnement et commercialisation notamment); ouverture des marchés; démocratisation de la vie publique et décentralisation administrative, etc. Les réformes entreprises ont transformé les conditions de production des agriculteurs et, en particulier, des exploitations familiales qui constituent la forme la plus répandue d'organisation de l'agriculture dans le monde. Les effets des réformes ont été particulièrement sensibles dans la plupart des pays d'Afrique, d'Amérique latine et d'Asie où se concentre la majorité de la population rurale mondiale.

Certains agriculteurs ont pu valoriser les opportunités offertes par ces évolutions et par l'ouverture des marchés. Tel n'est pas le cas des petits producteurs qui ont été confrontés à de nombreuses contraintes économiques (désorganisation de l'approvisionnement et de la commercialisation des produits, raréfaction et renchérissement du crédit, instabilité des prix, fortes concurrences pour l'approvisionnement des marchés à l'exportation et des marchés nationaux) auxquelles s'ajoutent bien souvent le manque d'infrastructures et un accès très limité aux services d'appui (information, formation, conseil).

Dans beaucoup de régions rurales du monde, les conditions de production se sont dégradées et les conditions de vie sont extrêmement difficiles. En témoignent notamment la forte prévalence de la pauvreté (selon le FIDA en 2010, plus de $70 \%$ des pauvres du monde étaient des ruraux), la précarité de la situation alimentaire mais aussi l'accentuation des processus migratoires, les transferts monétaires étant devenus indispensables à la survie de nombreuses familles.

C'est dans ce contexte que l'on observe, depuis 10 ou 15 ans, la naissance et la montée en puissance des OP, qui s'affirment, progressivement, en tant qu'acteurs à part entière du développement agricole et rural. Ce mouvement bénéficie d'une attention accrue et renouvelée de la part des acteurs institutionnels de la coopération pour le développement, même si cette attention s'accompagne parfois d'une attitude paternaliste voir manipulatrice.

Dans ce contexte général, les OP remplissent généralement trois rôles principaux, plus ou moins combinés, pour faire face aux difficultés que leurs membres rencontrent dans leurs activités d'agriculteurs.

- Fournir des services à leurs membres. Les OP permettent à leurs membres d'accéder à l'information, à la formation et aux innovations techniques (recherche, conseil agricole). Elles organisent aussi l'approvisionnement en intrants de leurs membres et la commercialisation de leurs produits. Les OP facilitent parfois l'accès au crédit. Elles 
peuvent également contribuer à une meilleure gestion des ressources naturelles (eau, pâturages, forêts, etc.).

- Améliorer le pouvoir de marché des producteurs, c'est-à-dire les mettre en position plus favorable pour commercialiser ou acheter des produits. En s'engageant dans les OP, les producteurs renforcent leur pouvoir de négociation avec les autres acteurs économiques (fournisseurs, industriels, commerçants). Les OP peuvent contribuer de façon active à la définition et à la mise en ouvre des nouvelles formes de coordination au sein des filières agricoles ou d'élevage.

- Influer sur les processus de prise de décision publique. Cette influence, exercée grâce aux OP, peut jouer aux échelons local, national, sous-régional, et international. Il s'agit pour les membres des OP de promouvoir des politiques agricoles et rurales ou des projets qui prennent en compte les spécificités des agricultures familiales ainsi que leur rôle central dans la réduction de la pauvreté et l'équilibre des sociétés.

Regardons maintenant de plus près la diversité des formes d'organisation des producteurs; c'est une étape indispensable pour déterminer les actions qui seront les plus pertinentes pour les renforcer.

\section{La diversité des formes d'organisation des producteurs}

Malgré des différences considérables entre les pays, deux tendances globales peuvent être dégagées : la multiplication des groupes locaux et le renforcement des dynamiques fédératives.

\section{ID La multiplication des groupements locaux}

Le désengagement de l'État et la démocratisation, même relative, de la vie publique ont favorisé d'une part, l'émancipation d'organisations de base jusque-là sous la tutelle des pouvoirs publics et, d'autre part, la naissance ou la consolidation de groupes locaux très divers. En Afrique subsaharienne, des enquêtes mettent en évidence l'ampleur du phénomène : $65 \%$ des villages enquêtés au Sénégal (de Janvry et Sadoulet, 2004) comptent au moins un groupement et c'est également le cas de la grande majorité des villages au Burkina Faso. Le taux moyen d'appartenance formelle des ménages ruraux à au moins un groupement est de $67 \%$ au Sénégal et de $62 \%$ au Burkina Faso. Bien 
que fragmentaires, des données disponibles pour d'autres pays mettent en évidence une augmentation rapide du nombre des groupes locaux. Ainsi, au Bénin, dans le département du Zou et des Collines, entre 1987 et 2001, le nombre de groupements villageois est passé de 52 à 578; dans la même zone, les groupements féminins, inexistants à la fin des années 1980, étaient estimés à 250 en 2001 (Agoua et al., 2000). Des évolutions similaires sont constatées dans la plupart des pays de l'Afrique subsaharienne (Cameroun, Ghana, Kenya, Tanzanie, Tchad) (Banque mondiale, 2006).

Dans la plupart des pays d'Amérique latine, les groupes locaux se comptent aussi par milliers. C'est le cas dans les pays andins où coexistent des coopératives et des groupes locaux aux statuts très divers. En Équateur, par exemple, on dénombrait, déjà en 1993, près de 3000 organisations de base dont 640 associations de création récente (Santana, 1997), en 2003, la seule Fédération nationale des organisations paysannes, indigènes et noires (Confederación nacional de organizaciones campesinas, indigenas y negras, FENOCIN) revendiquait 1300 organisations locales membres regroupant 200000 familles réparties sur 18 provinces, etc. Les pays du cône sud-américain ont une forte tradition coopérative, liée, en partie, aux politiques de réforme agraire. Dans la plupart des pays, des coopératives coexistent avec des associations de producteurs; c'est par exemple le cas au Brésil, où l'associativismo est très vivant dans le Nordeste et dans le Sud du pays et se développe aussi en Amazonie, dans les Cerrados.

En Asie, on observe des situations contrastées selon les pays mais les évolutions semblent globalement convergentes. Les coopératives sont très nombreuses en Inde : 135000 coopératives, 150 millions de coopérateurs pour un chiffre d'affaire estimé à 3,5 milliards de dollars US en 1998 (Draperi, 2003). Des réformes importantes touchent les coopératives chinoises dont certaines s'autonomisent et de nouvelles formes d'organisations locales voient le jour, les Farmers' Professionnal Associations (FPA) bien que de façon encore très minoritaire (Banque mondiale, 2006). Au Vietnam, les évolutions du cadre juridique des coopératives ont généré de profondes transformations dans leur fonctionnement et ont été accompagnées aussi de la création de nombreux groupes d'agriculteurs volontaires (Bergeret, 2003). Les groupes locaux sont très nombreux en Indonésie, qu'il s'agisse de certains Kelempok Tani ${ }^{1}$ qui fonctionnent encore, de coopératives ou

\footnotetext{
${ }^{1}$ Il s'agit de groupements paysans mis en place à l'initiative du gouvernement indonésien pendant la période de l'Ordre Nouveau sous la présidence Soharto.
} 
de groupes réunissant des agriculteurs ou des pêcheurs (Bourgeois et al., 2003).

Malgré la diversité des situations, on peut en général distinguer trois grandes catégories de groupement selon leurs objectifs ou leur constitution :

- les groupements spécialisés liés à une filière et qui assurent des fonctions économiques en matière d'approvisionnement, de commercialisation, de crédit, mais aussi des fonctions de conseil, d'information, de formation. Il peut s'agir d'organisations mises en place par les projets de développement antérieurs aux réformes et dont le statut et les activités ont évolué, ou d'organisations qui se sont créées après le désengagement de l'État;

- les groupements à vocation multisectorielle qui s'efforcent de prendre en compte la diversité des activités de leurs membres, euxmêmes très rarement spécialisés dans une production. Ils se sont souvent développés pour faire face aux carences des investissements publics ou de l'aide au développement. Dans certains cas, des intervenants extérieurs (ONG, projets) leur ont fait jouer des rôles proches de ceux des collectivités locales en émergence;

- les organisations qui regroupent des catégories sociales particulières (femmes, jeunes) et dont les activités sont souvent définies a posteriori comme moyen d'atteindre les objectifs économiques et sociaux des catégories concernées.

Dans beaucoup de situations pourtant très différentes, on constate donc des traits convergents dans ces dynamiques de création (illustrées par l'encadré 1) et de multiplication des groupements locaux.

Les groupements et associations ont des intensités d'activité très variables : certains groupements ont des résultats techniques et économiques significatifs. Mais il existe aussi des organisations qui, malgré une réelle base sociale, «tournent à vide» faute d'idées, d'appuis techniques ou de ressources adéquates. Il existe enfin des groupements plutôt liés à des stratégies opportunistes de quelques leaders locaux. Dans tous les cas, l'activité des groupements et leurs performances techniques et économiques, souvent irrégulières, dépendent en grande partie de leur capacité à nouer des relations avec l'extérieur. Cette capacité varie considérablement selon les cas : elle est plus importante pour les groupements liés à une filière jugée stratégique (cacao, riz, coton, etc.) pour l'économie nationale et qui bénéficieront de ce fait de programmes d'appuis extérieurs. Les groupements isolés, souvent 
liés à des filières moins bien considérées, ont généralement beaucoup de difficultés à développer leurs activités dans la durée.

Certains groupements locaux bénéficient d'appuis techniques et financiers extérieurs qui influent sur leur structuration. Ces appuis sont apportés par les administrations locales (ministères de l'élevage, de l'agriculture), par des ONG ou à travers des projets soutenus par l'aide publique au développement.

\section{Encadré 1. ONG, bailleurs de fonds et organisations de producteurs}

De nombreuses ONG ont incité à la création de groupements locaux pour acheminer les aides aux populations ou pour faciliter la gestion de ressources communes $($ eau,...). Dans les années 1992 à 2005, des projets d'appui à la professionnalisation de l'agriculture soutenus par le ministère français des Affaires étrangères (MAE) ont favorisé la structuration de groupements par filière comme au Cameroun par exemple. Des programmes d'appui de l'Agence française de développement (AFD) aux filières cotonnières d'Afrique francophone ont renforcé la spécialisation des organisations de producteurs : des groupements de producteurs de coton (GPC) ont remplacé progressivement les associations villageoises (au Mali) ou les groupements villageois (au Burkina Faso) qui assuraient des fonctions d'intérêt général (dispensaires, écoles, infrastructure) avec les ressources collectives générées par le coton. De nombreux groupements créés plus récemment, à l'initiative des producteurs ou sous l'impulsion d'interventions extérieures, sont eux aussi spécialisés dans une filière (Ghana, Ouganda, Cameroun, Mali, etc.). De façon générale, la plupart des bailleurs de fonds mettent l'accent sur le renforcement des activités économiques des organisations de base, ce qui va souvent de pair avec une spécialisation accrue des groupements et une segmentation des groupements et des associations pré-existants. D'autres projets de coopération, comme la coopération suisse en Afrique de l'Ouest, ont adopté d'autres stratégies en misant sur des leaders locaux et en accompagnant l'essor de leurs organisations pendant plusieurs décennies.

\section{Les dynamiques fédératives}

Ce foisonnement d'organisations locales de producteurs s'est accompagné d'un mouvement de regroupement, plus ou moins consistant selon les pays et les régions. En effet, les groupements locaux isolés peinent à tirer bénéfice de l'action collective engagée par leurs membres. Le regroupement dans des organisations plus vastes (unions, 
fédérations, réseaux d'organisations) leur permet souvent d'améliorer l'impact de leurs activités.

Afin de mieux atteindre leurs objectifs, les groupements locaux peuvent ainsi s'associer au sein d'unions locales. Certaines de ces unions se fédèrent dans des ensembles (fédérations) pouvant regrouper jusqu'à plusieurs centaines de groupements. Ces fédérations peuvent ellesmêmes se rassembler dans des fédérations plus larges, à l'échelle nationale, souvent désignées par le terme d'organisations faîtières.

Ces dynamiques répondent en général à une logique de communauté d'intérêts ou de problèmes partagés. En Afrique subsaharienne, des organisations fédératives se sont ainsi construites à l'échelon régional et national autour des principales filières (coton, cacao, riz, élevage, pêche, etc.) ou autour de l'épargne et du crédit (au Bénin par exemple); elles regroupent aussi des organisations régionales à vocation générale (c'est le cas de la FONGS-Action Paysanne, Fédération des $\mathrm{ONG}^{2}$ du Sénégal). Le regroupement des fédérations, de nature et d'origine très diverses, dans des coordinations nationales (appelées également plateformes) tend à se généraliser en Afrique subsaharienne (Kenya, Tanzanie, Sénégal, Tchad, Guinée, etc.) même s'il rencontre d'indéniables obstacles dans certains pays. En tout état de cause l'existence de structures - organisations faîtières, plateformes ou coordinations nationales - ne garantit pas forcément qu'il existe un mouvement paysan qui porte ces structures.

\section{ID Qu'est-ce qu'un mouvement paysan?}

À propos de la dynamique d'organisation des ruraux en Afrique à la fin des années 1980, Gentil et Mercoiret (1991) se sont interrogés sur l'existence de mouvement paysan en Afrique noire. Ils suggéraient alors cinq critères pour caractériser un mouvement paysan :

- une autonomie financière et intellectuelle;

- des objectifs conscients et explicites;

- des relations significatives de ce mouvement avec l'État ou les acteurs de la société civile;

- une taille et un poids économique et politique suffisants;

- une organisation interne établie.

${ }^{2}$ Les ONG membres de la FONGS sont des OP et se distinguent des ONG au sens courant ou ONG d'appui (voir tableau 1 et encadré 1). 
Ces auteurs ont conclu à l'absence de mouvement paysan dans cette région tout en soulignant les facteurs positifs annonciateurs d'une construction de ce mouvement ${ }^{3}$.

La décennie des années 1990 marque un tournant important dans la structuration des ruraux africains avec l'émergence de fédérations de coordinations nationales qui constituent aujourd'hui les composantes d'un véritable mouvement paysan en Afrique subsaharienne. Au début des années 2010, on peut légitimement dire qu'il existe un ou plutôt des mouvements paysans en Afrique. Il s'agit principalement du Réseau des Organisations paysannes et de producteurs de l'Afrique de l'Ouest (ROPPA) constitué en 2000, et qui regroupe des organisations paysannes de 10 pays de la sous-région. Il existe aussi la Fédération des agriculteurs d'Afrique de l'Est (Eastern Africa Farmers Federation, EAFF, créée en 2001), la Plateforme régionale des Organisations paysannes d'Afrique centrale (PROPAC, créée en 2004) et la confédération des unions agricoles d'Afrique australe (Southern African Confederation of Agricultural Unions, SACAU, créée en 1992). Ces grands réseaux régionaux se sont regroupés dans une organisation continentale, la Plateforme panafricaine des agriculteurs (Pan African Farmers Forum, PAFFO). Par ailleurs, des producteurs spécialisés se constituent également en organisation à l'échelle d'un continent avec par exemple la création en 2005 de l'Association des producteurs de coton africains (APROCA).

En Amérique latine, les crises et les mutations qu'ont connues les agricultures familiales ont diversifié de façon considérable les situations des familles rurales. Les grandes centrales syndicales ont dû s'adapter aux demandes très variées qui ont résulté de ces changements et ont parfois connu des réorientations importantes dans leurs programmes et leurs modes d'action. Parmi les nombreuses évolutions qui ont eu lieu, on peut en signaler deux : d'une part la création d'organisations (régionales ou nationales) centrées sur la production et la commercialisation, dont la coordination nationale des organisations productrices de Café (Coordinadora Nacional de Organizaciones Cefetaleras, CNOC) au Mexique en offre un exemple (Celis Calbejas,

\footnotetext{
${ }^{3}$ Ces auteurs estimaient alors que, sauf au Sénégal, la situation ne permettait pas encore de parler de mouvement paysan et que même dans le cas d'un multipartisme naissant (Bénin, Niger et peut-être bientôt Mali), les organisations paysannes n'ont pas été capables de faire entrer les problèmes des paysans, qui représentent pourtant la majorité de la population, dans le champ des enjeux politiques. Mais le rôle économique des organisations paysannes s'accroît et se diversifie, et les activités ne se cantonnent plus à des secteurs marginaux. Il n'y a pas encore de mouvement paysan dans la plupart des pays mais des amorces et des potentialités existent (Gentil et Mercoiret, 1991).
} 
2000) et, d'autre part, le renforcement des organisations indigènes qui ont notamment introduit la question culturelle dans le débat public. On observe aussi la création d'organisations sous-régionales, comme par exemple la coordination des organisations de producteurs du Mercosur (Confederación de Organizaciones de Productores Familiares del Mercosur, COPROFAM).

À noter enfin que l'importance, pour l'avenir des ruraux, des débats qui ont lieu à l'échelon international, a conduit à des rapprochements entre les organisations de différents continents, et, souvent, à leur adhésion aux deux fédérations internationales que sont la Via Campesina et la FIPA4.

La diversité des OP est grande (pour la lecture et l'interprétation de cette diversité, voir chapitre 9). Elle résulte de l'histoire et tient à la fois à la forme des organisations, aux actions qu'elles mènent et aux partenaires avec qui elles travaillent. La multiplication des organisations locales et leur articulation dans des dynamiques fédératives débouchent sur un paysage souvent complexe. Au-delà de leur diversité, nous allons voir que les rôles joués par les OP se recoupent et répondent à des enjeux qui n'intéressent pas seulement leurs membres ni même les ruraux mais également les États et les organismes impliqués dans le développement. Ceci justifie donc des actions en faveur du renforcement des OP.

\section{Pour aller plus loin}

Rapport sur le développement dans le monde 2008 (World development report 2008, téléchargeable sur le site http://documents.banquemondiale.org)

De Schutter O., 2010. Comment détruire la paysannerie mondiale de manière responsable? Project Syndicate, Bruxelles téléchargeable sur http://www.project-syndicate.org/commentary/ responsibly-destroying-the-world-s-peasantry/french

\footnotetext{
${ }^{4}$ La FIPA (Fédération internationale des producteurs agricoles), créée en 1947, a fait faillite en 2010. Une dynamique de coordination pour reconstruire une nouvelle structure internationale est actuellement animée par le COPAC (Comité des Organisations professionnelles agricoles de l'Union Européenne à Bruxelles). La Via Campesina a été créée en 1993 et regroupe 150 organisations dans 70 pays du monde (200 millions de paysans).
} 



\section{$\sqrt{2 .}$ Quels rôles jouent les organisations de producteurs?}

Cette question mérite d'être posée, car les OP ont à leur actif des réussites indéniables qui ne sont pas toujours reconnues à leur juste mesure, mais aussi parce qu'elles rencontrent des difficultés qui sont souvent mal comprises. Elles doivent donc encore relever de nombreux défis, et, pour y faire face, renforcer leurs capacités techniques et stratégiques. Lors de l'atelier organisé en 2006 par le MAE, le MAP et le FIDA 71 pour préparer le rapport de la Banque mondiale sur le déve-
loppement dans le monde (Mercoiret et al., 2007), les participants, s'appuyant sur les études préparatoires (Mercoiret et Minla Mfou'ou, 2006), ont souligné qu'il était pertinent de soutenir les OP dans ce sens car elles sont des acteurs essentiels des dynamiques de développement agricole et rural, et des représentants de la société civile dans des pays où la population est encore largement rurale.

\section{Des résultats réels mais parfois difficiles à apprécier}

À travers le monde, les OP ont de nombreuses réussites à leur actif en matière d'appui à l'innovation technique, de mise en place de services aux agriculteurs (information, formation, conseil agricole), d'organisation de l'approvisionnement, d'amélioration de l'accès au crédit et de la mise en marché des produits agricoles. Leur capacité à influer sur les centres de décision politique s'accroît, même si leur impact reste variable et si les résultats de certaines négociations avec les pouvoirs publics ne sont pas toujours suivis d'effet et sont parfois remis en cause. Des échecs sont aussi enregistrés et nombre d'actions débouchent sur des réussites partielles qui ne satisfont ni les adhérents, ni les responsables des organisations, ni leurs partenaires extérieurs. Ces résultats, même mitigés, constituent néanmoins souvent un progrès par rapport à la situation antérieure et l'activité des OP peut avoir des effets qui ne sont pas immédiatement visibles ou mesurables.

Plusieurs points expliquent pourquoi les résultats obtenus par les OP sont inégalement reconnus (tableau 2). En effet, selon la nature de ces résultats (technique, économique, changement de pratiques, influence 


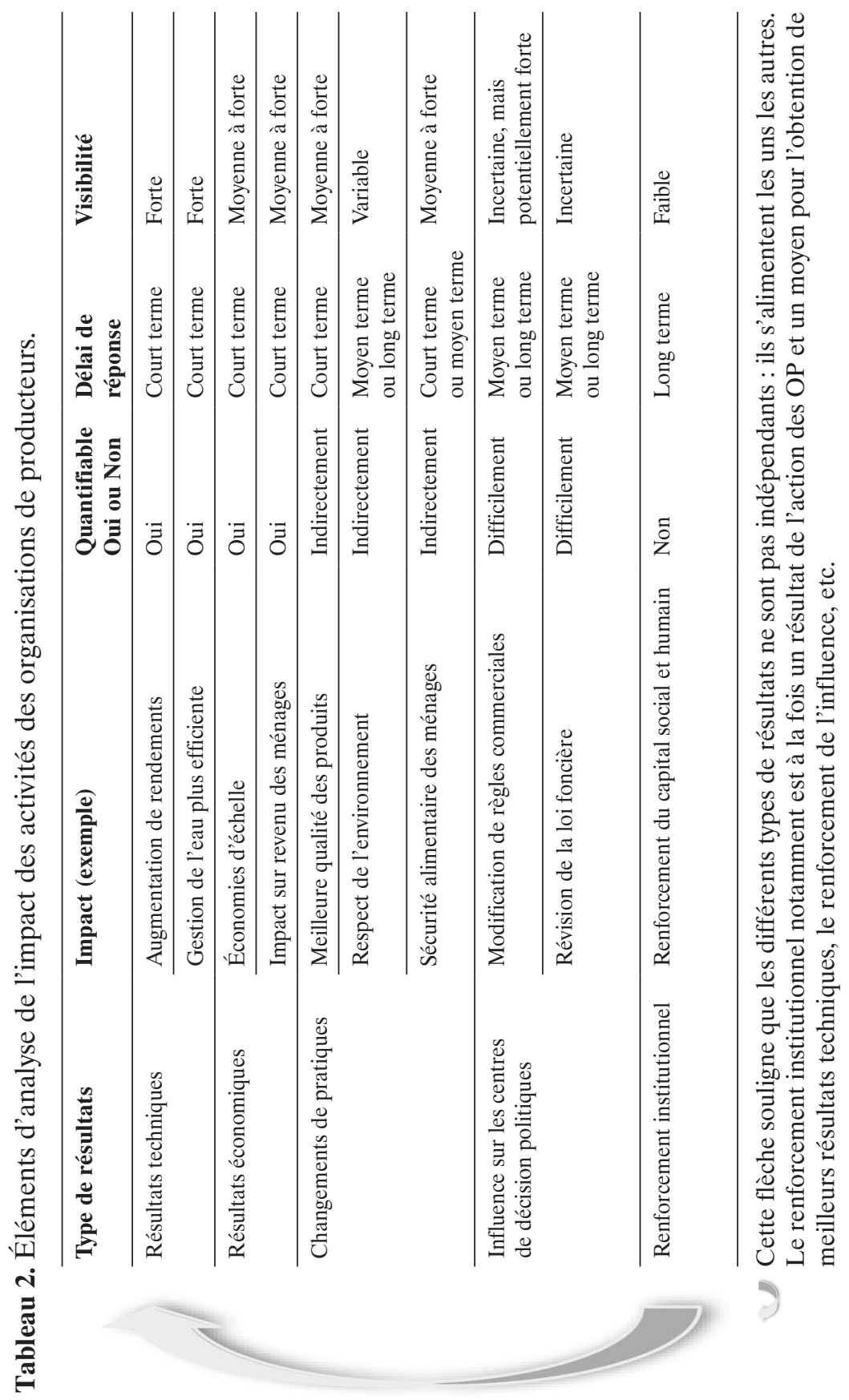


sur les politiques, renforcement institutionnel), l'impact n'est pas toujours quantifiable, voire peu visible, et ne se manifeste pas forcément rapidement. Un résultat à l'impact non mesurable peut cependant être très important, c'est le cas par exemple du renforcement institutionnel qui conditionne souvent les résultats techniques et économiques à moyen terme.

Des exemples montrent que les réussites d'envergure, quantifiables et appréciées, reposent toujours sur l'accumulation, pendant des périodes parfois longues, de nombreuses actions de faible portée, et dans tous les domaines. Prises séparément, elles paraissent insignifiantes mais, jour après jour, elles peuvent apporter des solutions de proximité aux problèmes concrets que rencontraient les ruraux et renforcer leur confiance dans l'efficacité de l'action collective et les liens entre les membres.

Les OP agissent dans un environnement économique et institutionnel caractérisé par de nombreuses contraintes qui freinent leurs initiatives et compromettent leurs résultats : inadaptation de certains cadres juridiques, manque de transparence qui caractérise nombre de transactions et de décisions, difficultés pour avoir accès au crédit, rareté et instabilité de l'offre de services, concurrences déloyales pour l'approvisionnement des marchés, soutiens et appuis très insuffisants (ou inexistants) des pouvoirs publics, etc. L'appréciation des résultats ne peut faire abstraction du contexte dans lequel ces résultats ont été obtenus et leur amélioration suppose aussi que des efforts soient faits pour rendre ce contexte plus favorable.

La contribution des OP aux équilibres sociaux est généralement peu prise en compte dans l'évaluation de leurs résultats du fait qu'elle est diffuse et difficilement quantifiable; elle est pourtant très importante et revêt deux dimensions principales.

- À travers l'expérience acquise au sein de chaque organisation et à travers les relations qu'elles établissent avec les acteurs extérieurs, les OP renforcent progressivement la confiance des ruraux dans leur propre potentiel. Elles créent des conditions favorables à l'apprentissage des producteurs pour que s'opèrent des changements mieux maîtrisés par les producteurs et contribuant à la construction de sociétés plus démocratiques.

- Le rôle des OP apparaît particulièrement important dans des sociétés confrontées à des transitions démographiques et économiques rapides. La dégradation des conditions d'existence se traduit dans beaucoup d'endroits par un exode rural massif qui concerne toutes 
les catégories de ruraux et en particulier les plus jeunes et les mieux formés d'entre eux. Dès lors que le secteur urbain n'est pas en mesure d'offrir des emplois en nombre suffisant et qu'il n'existe pas de filets sociaux à la hauteur du défi démographique, la migration internationale s'impose de plus en plus comme la seule solution possible. Force est de constater qu'il s'agit là d'une option peu réaliste et qui conduit à des situations parfois dramatiques. Par les actions qu'elles mènent, les OP contribuent, même modestement, à créer des alternatives : localement, elles stimulent l'innovation dans certains domaines, elles aménagent le cadre de vie et elles redonnent espoir. De plus, par leur action sur les politiques publiques, les OP s'efforcent de créer des conditions plus favorables aux initiatives des producteurs familiaux, concernant l'agriculture ou d'autres activités génératrices de revenus, et améliorent donc indirectement les revenus des familles.

\section{Les OP face à des défis nécessitant des capacités renforcées}

Ayant constaté les divers rôles joués par les OP, on pressent que le renforcement de leurs capacités constitue un enjeu déterminant pour leur essor. En effet, bien qu'elle soit souvent une solution, l'organisation est toujours un problème et les $\mathrm{OP}$ n'échappent pas à la règle, ainsi qu'en témoignent les nombreuses tensions qui émaillent leur vie et la lassitude que ressentent souvent leurs animateurs et leurs dirigeants.

\section{ID Les défis à relever}

Les OP doivent composer avec plusieurs impératifs qui constituent des enjeux pour leur viabilité d'autant plus que leurs membres vivent dans des conditions difficiles et que l'environnement ne leur est pas toujours favorable. Ladministration et les acteurs du développement ne jouent en effet pas toujours le jeu de leur renforcement et voient parfois dans les OP des rivales à leurs propres activités. Le schéma ciaprès résume la situation (schéma 1).

En plus d'apporter des solutions (1), même partielles et limitées, à certains des problèmes auxquels sont confrontés leurs membres, les OP (via leurs fédérations) sont appelées à jouer un rôle de représentation dans diverses instances de concertation (2) : ce sont leurs deux fonctions premières, ce qui ne veut pas dire que cela va sans 


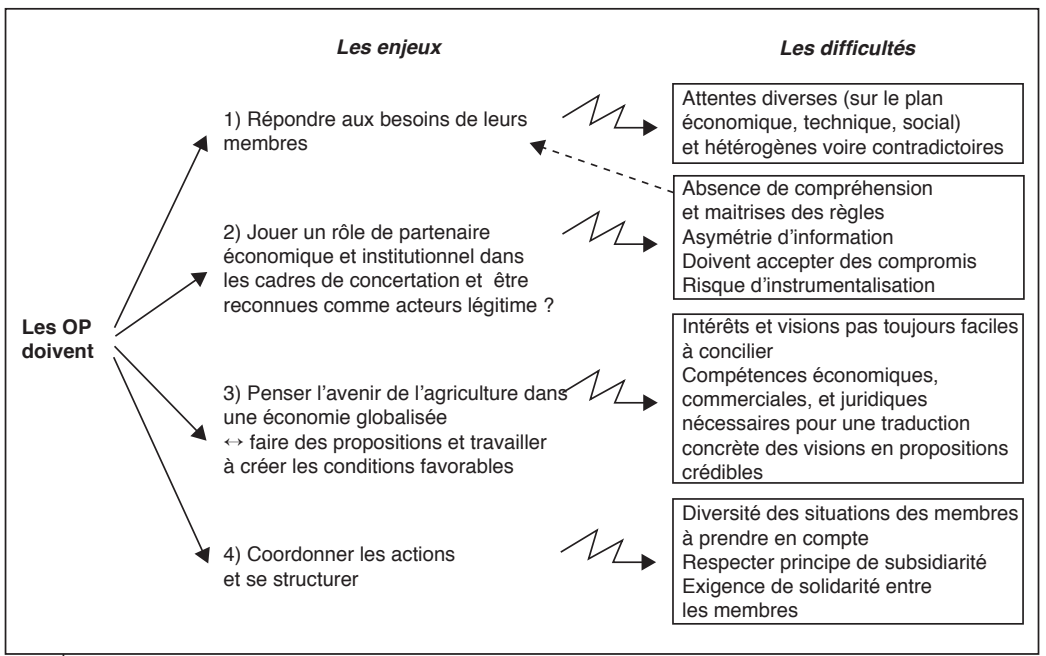

\section{Schéma 1.}

Problématique du renforcement de capacités des OP :

les défis à relever et les difficultés.

difficultés (voir parties 2 et 3). Mais la nécessité de développer une vision de l'agriculture à plus long terme (3), de travailler à la rendre réalisable et de la traduire en mesures concrètes est sans doute encore plus complexe, de même que le travail de coordination (4) et de structuration progressive, d'autant plus que les organisations sont souvent relativement jeunes.

Une difficulté supplémentaire vient du fait que ces quatre enjeux doivent être abordés simultanément car ils sont, de fait, interdépendants. La recherche de solutions durables à des problèmes urgents ou de court terme des producteurs suppose tôt ou tard des négociations avec d'autres acteurs et renvoie souvent à des mesures plus générales qui relèvent de niveaux de décisions supérieurs. Les positions défendues par les OP dans les différents cadres de concertation gagnent en cohérence quand elles s'appuient sur les références communes données par une vision stratégique à long terme. En outre, elles ne peuvent jouer le rôle que leurs membres attendent d'elles que si elles ont le pouvoir de proposition et de négociation que donne une forte structuration.

Progressivement, les partenaires du développement prennent conscience des défis auxquels sont soumises les OP, et ce d'autant plus que le rôle qu'elles ont à jouer est mieux reconnu. 
C'est notamment le cas de la Banque mondiale qui, dans son rapport sur le développement, de l'année 2008, assigne aux organisations de producteurs un rôle essentiel dans la modernisation de l'agriculture, au sens des gains de productivité et d'efficacité économique (encadré 2).

\section{Encadré 2. Cinq défis pour les organisations de producteurs}

Dans le rapport de l'année 2008 de la Banque mondiale «l'Agriculture au service du développement», le renforcement des OP et l'amélioration de leurs performances sont soulignés comme étant un des six objectifs principaux que devraient adopter les nouvelles politiques pour le secteur agricole. La Banque mondiale souligne le rôle clé joué par les OP dans la modernisation des agricultures des pays industrialisés. Cinq défis majeurs sont identifiés.

\section{Résoudre les conflits entre efficience et équité}

Beaucoup d'OP fonctionnent selon les normes des sociétés rurales traditionnelles (solidarité, inclusion) qui peuvent être incompatibles avec les exigences d'organisations professionnelles tournées vers les affaires. $\mathrm{Au}$ nom de la solidarité, elles peuvent soutenir la mauvaise performance des uns et devenir inefficaces. Elles ont des difficultés à exclure des membres qui ne respectent pas les accords.

\section{Gérer l'hétérogénéité de leurs membres}

Les OP sont amenées à représenter les intérêts de membres aux préoccupations très diverses. Comment s'assurer que les intérêts des petits agriculteurs, des femmes et des jeunes soient biens pris en compte?

\section{Développer des capacités managériales sur les filières à haute valeur ajoutée}

La globalisation et les filières internationales défient les capacités des OP pour fournir un accès aux marchés à leurs membres. Les responsables des OP doivent gérer des filières nationales et internationales de plus en plus sophistiquées avec des exigences de plus en plus fortes (organisation de l'offre, respect des normes). Les OP doivent devenir des organisations très professionnelles pour réussir sur des marchés où la compétition est intense.

\section{Participer aux négociations à haut niveau}

Les OP sont mieux représentées à la table des négociations sur les questions globales comme le commerce. Les responsables des OP ont besoin de personnes ressources (professionnels aux compétences techniques et de communication) pour participer à ces discussions techniques de haut niveau. 
En même temps, les fédérations internationales et les réseaux d'organisations doivent rester fidèles aux intérêts des organisations nationales et locales. Pour cela, des consultations régulières sont nécessaires à différents niveaux (local, régional et national) pour maintenir le flux de communication avec leurs membres.

\section{Gérer un environnement parfois défavorable}

Pour faire face à ces quatre défis, les OP ont besoin d'un environnement légal et politique qui leur soit favorable. Pour cela, les relations avec les pouvoirs publics doivent être améliorées et ceux-ci doivent être à l'écoute de ces nouveaux acteurs.

(extrait de Banque mondiale, 2007. L'agriculture au service du développement)

\section{IID Des capacités techniques et stratégiques à renforcer}

Face à ces nombreux défis, quelles capacités renforcer? Comment s'y prendre pour accompagner les OP? Quelle que soit l'OP concernée, de nombreux intervenants extérieurs développent des actions sur le registre du renforcement des capacités techniques et des capacités stratégiques. De quoi s'agit-il?

Par capacités techniques, on entend un ensemble de compétences nécessaires à la conduite des activités menées par l'OP ou au contrôle des activités dont elle confie la réalisation à des organismes extérieurs. Ces savoir-faire dans des secteurs précis (production, commercialisation) relèvent de la maîtrise d'ouvrage, c'est-à-dire de la capacité à gérer, en interne ou avec une institution partenaire, la conception, la conduite, l'évaluation et le contrôle d'une ou de plusieurs actions. Les capacités techniques sont le plus souvent mises en place ou renforcées à travers l'acquisition de connaissances dans le cadre de formations «classiques» (voir chapitre 6). Mais elles s'intègrent réellement dans le patrimoine des OP quand ceux qui les ont acquises (les producteurs par exemple) ont pu les mettre en application ou ont développé des échanges avec ceux qui les mettent en pratique (apprentissages).

Par capacités stratégiques, on entend les capacités qui permettent à une OP de définir et conduire une stratégie en prenant en compte son environnement (proche et lointain), les aspirations de ses membres et les diverses contraintes et opportunités auxquelles elle est confrontée. Ce type de capacités est rarement acquis par des formations proprement dites mais le plus souvent par des voyages, des contacts 
personnels et des expériences particulières. Ces capacités sont intimement liées aux caractéristiques personnelles des leaders. Elles dépendent également de leur capacité à entretenir, au sein de l'OP et à l'extérieur, la vision commune et les ambitions qui fondent son identité, ce qui permet de mobiliser les ressources humaines nécessaires aux activités. Dans cette catégorie des capacités stratégiques, on peut aussi citer les capacités organisationnelles qui sont celles qui permettent à l'organisation de «bien fonctionner»; dans ce domaine, les appuis apportés vont d'approches assez standardisées, privilégiant les modes organisationnels classiques (associatif, coopératif) à des approches plus complexes proposant aux responsables les outils de gestion de leur organisation (management, gestion des ressources humaines, réflexion stratégique).

Le renforcement des capacités des OP appelle des modalités diversifiées selon qu'il s'agit de renforcer leurs capacités techniques (conseil aux membres dans le domaine technique et économique, appui à la gestion, etc.) ou leurs capacités stratégiques (définition d'orientations et de programmes de travail, formulation de propositions et négociations, etc.) (voir chapitre 6).

En effet, le renforcement des capacités techniques peut passer par des interventions extérieures négociées (prestataires, projets), par la construction d'institutions contrôlées par les OP (centres de formation, centres de services pour le conseil de gestion par exemple) ou par une meilleure maîtrise de la contractualisation des services.

En revanche, pour renforcer leurs capacités stratégiques, les OP doivent pouvoir disposer d'une expertise indépendante qu'elles choisissent; l'indépendance de cette expertise est essentielle pour les aider à se positionner sur des dossiers importants (prise de participation dans des sociétés cotonnières, analyse des politiques agricoles nationales et des débats internationaux, etc.). Les OP doivent également avoir des moyens pour organiser leur information et leur communication internes, la consultation des membres, la réunion de leurs instances. Elles ont aussi besoin de moyens pour échanger avec d'autres organisations des pays voisins (sous-régions) et sur le plan international.

\section{Des arguments en faveur du renforcement des OP}

Si les partenaires directs des OP sont conscients de l'intérêt de leur renforcement, ce n'est pas encore le cas de tous les décideurs. Quatre 
types d'arguments peuvent pourtant être avancés pour justifier d'investir dans un appui aux OP.

\section{ID Les OP sont des acteurs à part entière du développement rural}

Il est peu productif et de plus en plus souvent impossible d'ignorer les OP, car comme nous l'avons déjà souligné au chapitre 1 , les OP jouent des rôles importants et croissants de trois types :

- pour reconstruire des services adaptés aux demandes et aux ressources des ruraux et en particulier des plus pauvres d'entre eux;

- pour améliorer le pouvoir de marché des producteurs ruraux en renforçant notamment leur pouvoir de négociation avec les autres acteurs économiques du secteur privé;

- pour influer sur les processus de prise de décision aux échelons local, national, sous-régional et international, afin notamment de promouvoir des politiques agricoles et rurales qui prennent en compte les spécificités des agricultures familiales, ainsi que leur rôle central dans la réduction de la pauvreté et l'équilibre des sociétés.

Le regroupement de ruraux dans des OP est à l'origine d'innombrables initiatives à travers le monde et de très nombreuses réussites. En rendant possible l'action collective, il permet en effet de dépasser les contraintes que constituent à la fois une production très dispersée et atomisée dans des unités le plus souvent de petite taille et les capacités très réduites de chaque famille rurale à améliorer de façon significative ses conditions de production et d'existence et à faire entendre sa voix. Les résultats obtenus sont certes encore de portée variable; ils constituent cependant une avancée considérable et de nombreux exemples montrent que l'action collective, en renforçant la confiance des ruraux dans leur propre potentiel, facilite les changements maîtrisés qui sont indispensables en milieu rural.

\section{ID Les OP sont des acteurs légitimes des stratégies de réduction de la pauvreté}

Bénéficiant d'une reconnaissance globale accrue de la part des acteurs extérieurs, les OP sont l'objet d'interrogations qui portent sur leur volonté et leur capacité à jouer un rôle important dans les stratégies de réduction de la pauvreté. Deux questions sont souvent posées par 
des acteurs du développement (États, bailleurs de fonds) : les OP regroupent-elles réellement les pauvres? Lorsqu'ils en sont membres, les pauvres peuvent-ils y faire entendre leur voix?

Trois points peuvent à cet égard être soulignés.

- Il existe à l'évidence des OP qui sont progressivement amenées à sélectionner leurs adhérents et adoptent une dynamique d'exclusion (voir également le chapitre 5) sur des critères de spécialisation, de performances techniques et économiques. Plusieurs études mettent cependant en évidence que, dans leur immense majorité, les OP incluent des membres divers qui appartiennent pour la plupart aux catégories sociales les plus vulnérables.

- Les responsables des organisations fédératives, notamment à l'échelon national et supranational, ne sont pas toujours considérés comme représentatifs. Nombre de facteurs les distinguent en effet souvent des «paysans moyens». Cela est vrai dans les pays du Nord comme dans les pays du Sud et ne remet pas forcément en cause la légitimité sociale des responsables qui incarnent souvent l'objectif vers lequel tend l'organisation. Les responsables sont en quelque sorte en avance sur leur temps et contribuent à entraîner les adhérents dans un cheminement qu'ils ont eux-mêmes entrepris et qu'ils poursuivent.

- Enfin, et dès lors que les contraintes et les attentes diversifiées des différentes catégories d'adhérents sont prises en compte, la mixité des membres en termes de genre, de génération et de conditions socio-économiques constitue un atout. Du fait de cette mixité, tous les membres, même les plus pauvres, bénéficient en effet d'un large éventail d'opportunités. À l'inverse, des organisations spécialisées par catégorie peuvent renforcer la rigidité de la stratification sociale.

\section{ID L'implication des OP facilite la rénovation des politiques publiques}

Lexpérience dans les pays du Nord et dans certains pays du Sud montre que l'efficacité des politiques publiques dépend premièrement de leur adaptation au contexte particulier dans lequel elles s'appliquent - le sur mesure est toujours préférable aux modèles normatifs prêts-à-porter - et de leur acceptation et appropriation par les catégories sociales concernées, et deuxièmement de leur contenu technique intrinsèque ou de leur conception propre. 
La définition des politiques publiques pour le milieu rural est certes une prérogative des pouvoirs publics aux différents niveaux auxquels elles s'élaborent. Cependant, leur efficacité s'accroît quand elles reposent sur des compromis négociés entre les différents acteurs concernés et lorsque ceux-ci sont associés à leur mise en œuvre selon des modalités concertées. Les OP ne peuvent prétendre à l'exclusivité du dialogue avec les pouvoirs publics qui concerne aussi les collectivités territoriales, le secteur privé marchand, les consommateurs, etc. Regroupant les producteurs ruraux, très souvent encore largement majoritaires, elles ont cependant un rôle indispensable et central dans la représentation des intérêts de leurs membres et dans l'application des décisions prises.

\section{ID Les OP participent à l'approfondissement de la démocratie}

Dans de nombreux pays la réduction de la pauvreté et plus largement le développement économique et social sont inséparables de l'amélioration des différentes formes de gouvernance; des efforts importants sont déployés dans ce sens par de nombreuses agences de financement et de coopération pour le développement. Une amélioration de la transparence, dans les prises de décision et dans la gestion, et une véritable pratique de l'accountability ${ }^{5}$ des décideurs vis-à-vis de la population supposent un renforcement de la société civile dont on espère l'émergence et toujours la consolidation. Force est de constater que dans les pays où la population rurale est importante et parfois majoritaire, les OP en font nécessairement partie et peuvent constituer des corps intermédiaires, des contre-pouvoirs efficaces, même s'il est évident qu'elles ne peuvent, en la matière, prétendre à l'exclusivité.

Les OP jouent donc de nombreux rôles aussi bien pour répondre aux besoins de leurs membres que vis-à-vis de la société et de l'État. C'est pourquoi il est justifié de soutenir leur renforcement. Celui-ci concerne à la fois la durabilité des organisations (liée principalement à des caractéristiques internes) et leur efficacité dans leurs relations avec l'extérieur. Des principes et des pistes d'action pour appuyer les OP dans ces deux domaines sont proposés respectivement dans les deuxième et troisième parties de ce manuel.

\footnotetext{
${ }^{5}$ Terme non traduit en français : il combine l'idée de « responsabilité » et celle de « rendre compte».
} 



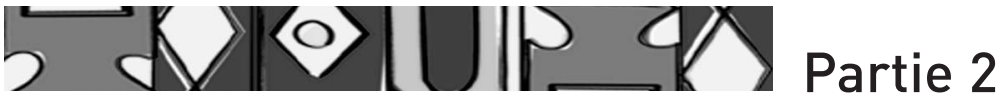

\section{Renforcer les organisations de producteurs de l'intérieur}

Parmi les questions à régler par les organisations de producteurs pour remplir les rôles et fonctions qui leur sont dévolus, certaines sont exclusivement de leur responsabilité : il s'agit de tout ce qui concerne les liens entre l'organisation et ses membres et les moyens de les entretenir ou de les renforcer. C'est l'objet de cette deuxième partie qui est composée de quatre chapitres portant sur : le choix d'une structure appropriée (chapitre 3); la définition des orientations à moyen et long termes (chapitre 4); le fonctionnement quotidien (chapitre 5); la question transversale du renforcement des capacités au sein des organisations (chapitre 6). 



\section{$\sqrt{3 .}$ Établir le cadre de l'organisation de producteurs}

Quelles règles se donner et pourquoi? La question se pose souvent au début de la vie d'une organisation quand il s'agit d'en fixer la structure. Cette structure conditionne en partie l'efficacité des actions et la marge de manœuvre dont disposent les responsables. Elle est également une des composantes de l'image de cette organisation. Si la structure formelle fixe en quelque sorte le cadre, il ne faut pas négliger une autre dimension de l'identité des organisations : il s'agit des systèmes de valeurs, parfois divers, portés par les membres et les responsables, qui vont peser sur les relations internes ainsi que sur les façons d'agir et de prendre des décisions. La notion de gouvernance recouvre plus ou moins explicitement l'ensemble de ces éléments.

\section{La structure d'une organisation}

Les formes d'organisations sont multiples. Pour les appréhender, plusieurs entrées sont possibles. Pour représenter la structure d'une organisation, on pense généralement à des organigrammes qui définissent les différents organes et les éléments de l'organisation, leurs rôles et les relations de pouvoir entre ces entités. Mais cette représentation est plus proche du monde des entreprises et des administrations que de celui des OP. Elle est toutefois très utile pour caractériser et réfléchir la structure des grandes organisations en particulier celles combinant plusieurs logiques.

Une OP peut se structurer sur des fondements divers : par fonctions (service intrants, service commercialisation par exemple), par produits (producteurs de riz, producteurs de coton, éleveurs, maraîchers, etc.), par entité géographique ou administrative, ou par une combinaison de ces critères (en prenant en compte l'organisation géographique et la production : voir exemple de la Fédération des unions des producteurs au Bénin, schéma 2). Le choix dépend de la taille et des objectifs de l'organisation. 


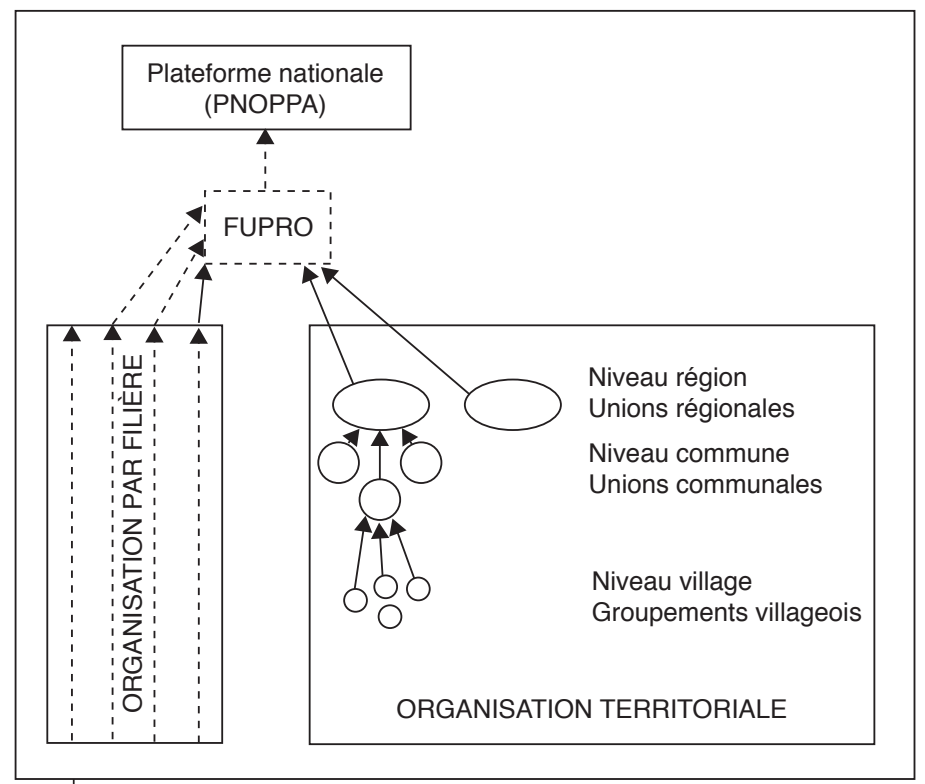

Schéma 2.

Structuration actuelle de la Fédération

des unions des producteurs (FUPRO) au Bénin.

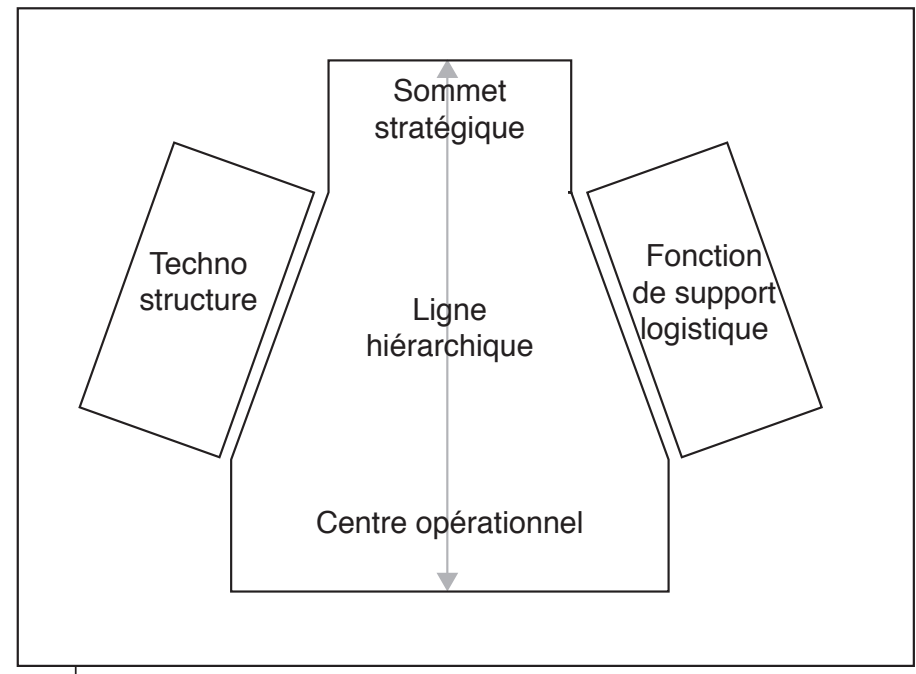

Schéma 3.

Les composantes d'une organisation. 
Henry Mintzberg, un chercheur canadien ayant passé sa vie à étudier toutes sortes d'organisations, propose de décrire la structure d'une organisation par la combinaison de plusieurs composantes (Mintzberg, 1982 et 1989) (schéma 3) :

- le sommet stratégique est constitué des organes politiques ou décisionnaires qui élaborent la stratégie et prennent les décisions à long et moyen termes. C'est la tête d'une organisation. Dans le cas d'une OP, c'est souvent un bureau, généralement élu et mandaté par l'assemblée générale, et les personnes qui assurent la fonction de coordination, qu'elles soient élues ou salariées, il peut s'agir d'un directeur, secrétaire général, coordinateur ou gérant dans une entreprise. Le mode de désignation peut être indirect dans les très grandes organisations, ou dans les organisations à structure complexe, comme dans le cas de la FUPRO dont le bureau est composé de représentants par filières et de représentants territoriaux;

- le centre opérationnel traduit ces orientations et met en œuvre les activités. C'est le cour de l'organisation, ce qui fait sa substance et sa réalité. Par analogie, dans le cas d'une organisation de producteurs de coton, le centre opérationnel pourrait être assimilé aux producteurs. Même s'ils sont de fait indépendants, c'est souvent sur leur travail, tout au long de l'année, dans les champs, que reposent à la fois la raison d'être et la production de ressources de l'organisation des producteurs de coton;

- dans les grandes organisations, il existe en général une ligne hiérarchique. C'est l'ensemble des personnes qui font le lien entre le sommet stratégique et le centre opérationnel, par exemple des responsables de zones. C'est souvent l'équipe des salariés, quand elle existe, qui joue ce rôle, mais aussi des membres bénévoles;

- les personnes et les services qui travaillent à améliorer le fonctionnement de l'organisation en elle-même constituent ce qu'on appelle la technostructure (comptabilité, formation, recherche);

- les supports logistiques qui fournissent des services internes à l'organisation (conseil juridique, communication, relations publiques, etc.).

Dans beaucoup de cas, la technostructure et les supports logistiques sont assurés par les salariés, par des membres bénévoles ou plus ou moins indemnisés. Ces fonctions peuvent également, totalement ou en partie, être assurées par des personnes ou services extérieurs à l'organisation qui apportent leur compétence sous forme de prestation.

Henry Mintzberg inclut dans la structure d'une organisation, un sixième élément, moins matériel, qui est l'idéologie de l'organisation ou la 
culture d'entreprise. Cette composante se nourrit des traditions, des normes et des valeurs dominantes, des croyances et des convictions de l'organisation (voir ci-après «Identités et systèmes de valeurs»). Dans le cas d'une OP, cet élément, représentant l'idéologie ou la culture, de nature différente des premiers, est primordial pour comprendre le fonctionnement et l'évolution de l'organisation.

La structuration d'une organisation répond à une double nécessité : la division du travail et la coordination entre les tâches. En effet, au fur et à mesure de l'essor de ses activités, et parfois très tôt dans la vie de l'organisation, les tâches à remplir se multiplient et sont réparties en fonction des compétences, des intérêts, des disponibilités des uns et des autres (membres ou salariés). Mais, dès qu'il y a division du travail, les questions de la coordination, de l'information et de la prise de décision se posent; elles peuvent représenter des sources de tension au sein des organisations.

Parmi les OP, on rencontre principalement des structures simples, composées seulement d'un sommet stratégique et d'un centre opérationnel : c'est le cas de la plupart des OP de base. On peut aussi rencontrer des structures plus complexes pour les quelques OP disposant de moyens minimum de fonctionnement. Il existe aussi des OP constituées d'un ensemble de structures simples liées entre elles dans le cadre d'une structure fédérative (fédération, union), d'un réseau ou d'une plateforme (encadré 3).

La structure d'une OP n'est pas définie une fois pour toutes. La structuration résulte souvent d'une construction progressive et d'ajustements face à un environnement qui évolue et qui présente de fortes contraintes. Cette structuration s'inscrit dans une trajectoire qu'il est intéressant d'identifier et de comprendre car elle conditionne les choix stratégiques et le fonctionnement de l'organisation ainsi que les modes d'accompagnement utiles et acceptables. Il faut aussi préciser qu'une organisation peut garder le même statut juridique et changer de structure, tout simplement par élargissement du nombre de membres par exemple, ou par développement de nouveaux secteurs d'activités. Ces changements de structure peuvent correspondre à des évolutions postérieures à des périodes de crises que les organisations ont cherché à dépasser.

Une trajectoire d'évolution d'une organisation se distingue par plusieurs aspects : 


\section{Encadré 3. Quelques notions sur la structure}

Ce qui est important quand on analyse la structure d'une organisation donnée, c'est de comprendre qui sont ses membres et quelles sont les relations entre les différents niveaux. En effet, cela permet de caractériser la répartition des fonctions, des actions et des décisions et de regarder s'il existe une certaine subsidiarité entre les différents niveaux. La subsidiarité suppose que tout échelon supérieur s'interdit de réaliser une tâche qu'un niveau inférieur pourrait réaliser. Cela permet aussi d'identifier les mécanismes d'accountability (idées de «responsabilité» et de «rendre compte»), autrement dit, savoir qui rend compte à qui sur quel sujet, ce qu'on appelle les aires de gouvernance.

On distingue souvent (voir le schéma 2, Organisation de la FUPRO au Bénin) : - les groupements ou organisations de base, dont les membres sont des individus;

- les unions dont les membres sont des groupements;

- les fédérations qui regroupent des unions ou des groupements ou des organisations faîtières;

- les confédérations, les réseaux.

Il n'existe pas de définition unique de ces différents termes qui peuvent recouvrir des réalités différentes selon les pays, les époques ou les organisations.

En général, les relations qui existent au sein d'un réseau sont plus souples, moins formelles que celles qui existent au sein d'une union ou d'une fédération dont le mode de gouvernance est plus centralisé. En général, une union constitue un centre de décision unifié alors qu'une fédération préserve l'autonomie de décision de ses membres.

- la croissance plus ou moins marquée du nombre de membres, avec souvent des périodes de stabilité qui alternent avec des périodes de forte croissance ou, au contraire, de baisse des effectifs;

- la spécialisation sur une fonction particulière ou, au contraire, la diversification des activités avec une augmentation du nombre de fonctions assurées par l'organisation (voir chapitre 7);

- la croissance ou l'ouverture avec un élargissement à de nouvelles catégories de membres ou une extension géographique;

- une forte centralisation ou au contraire une décentralisation des activités.

Un travail de représentation de la trajectoire d'une organisation se révèle souvent un support très intéressant dans la démarche de réflexion 
stratégique (voir chapitre 4). Pour cela, il est utile de commencer par replacer les événements marquants, positifs ou négatifs, souvent qualifiés de fondateurs, de repérer les changements importants que les membres de l'organisation ont gardés en mémoire. Ceci constitue l'axe de temps, par rapport auquel on place les autres évolutions.

Il n'y a pas de structuration parfaite et une organisation dynamique cherche généralement à adapter sa structure aux ambitions de ses membres, aux objectifs qu'ils se sont fixés et aux évolutions du contexte.

\section{L'identité et les systèmes de valeurs}

Les organisations sont porteuses d'identités et de systèmes de valeurs qui régissent les rapports et facilitent - ou compliquent - l'entente entre les membres de l'organisation. Des valeurs internes bien identifiées et partagées facilitent la gestion.

Les systèmes de valeurs interviennent notamment dans :

- les règles du partage des ressources, qui se réfèrent aux notions d'équité et d'égalité;

- la gestion du temps;

- les rapports hiérarchiques et la gestion du pouvoir;

- la façon de prendre des décisions : consensus, vote à la majorité;

- la perception des tensions et conflits.

Plusieurs systèmes de valeurs peuvent coexister dans une OP, notamment entre les différentes catégories d'acteurs qui interviennent au sein de l'organisation : producteurs et salariés par exemple ou entre membres de différentes régions dans le cadre d'une grande organisation. Ces décalages existent aussi souvent entre les membres de l'organisation et les partenaires extérieurs qui veulent l'accompagner, ou les administrations qui cherchent à les appuyer ou à les encadrer. C'est le cas par exemple quand une OP veut promouvoir la solidarité et qu'on lui oppose le principe libéral des vertus de la concurrence.

Certaines crises internes dans le fonctionnement d'une organisation peuvent être liées à la difficulté de trouver des compromis entre ces différentes valeurs, tensions souvent exacerbées par des conflits de personnes. Ces crises proviennent aussi de l'inadaptation des structures aux nouveaux défis et activités des OP.

C'est une force pour une organisation de pouvoir se référer sans ambiguité à des principes admis par tous, notamment dans les périodes 
de crise. C'est pourquoi ces principes fondateurs sont généralement rappelés explicitement dans les statuts, qui constituent les textes de référence, que se donne l'organisation. Là encore, ces principes peuvent évoluer, il convient alors d'adapter les textes en conséquence pour éviter des décalages entre les principes affichés et les pratiques, sources d'incompréhensions et de conflits possibles.

Cependant les textes statutaires ne suffisent pas. C'est une dimension essentielle du travail des leaders que d'incarner ces principes et de les faire vivre, non seulement dans les discours mais également dans les actes, individuels et collectifs.

Le sentiment d'appartenance ressenti par ses membres est déterminant pour la solidité et le dynamisme d'une organisation. Il dépend de nombreux facteurs parmi lesquels la qualité des services reçus n'est qu'un élément. Ce sont aussi les valeurs partagées, l'existence d'un projet commun débouchant sur l'action, qui renforcent ce sentiment d'appartenance, essentiel à la pérennité de l'organisation et à sa capacité à surmonter les crises. D'où l'importance, pour les responsables principalement, de consacrer le temps et les moyens nécessaires à forger cette identité partagée.

\section{Pour aller plus loin}

Amblard H., Bernoux P., Herreros G., Livian Y.F., 2005. Les nouvelles approches sociologiques des organisations. Le Seuil, Paris. 296 p. 



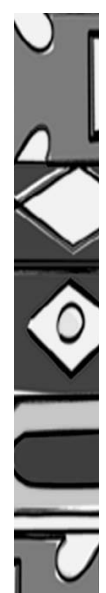

\section{Définir les orientations d'une organisation de producteurs et les respecter}

La vie d'une OP est faite de grands événements et de quotidien. Dans un environnement incertain, où les sollicitations sont nombreuses et parfois contradictoires, il n'est pas facile pour une organisation de définir des objectifs à moyen et long terme puis de garder une ligne d'action cohérente et lisible par ses membres, ses partenaires et ses interlocuteurs. C'est à cela que sert la réflexion stratégique, qui sous-tend à la fois les choix à long et moyen termes (plan stratégique) et leur application opérationnelle à plus court terme (plan d'action). Lanalyse des résultats obtenus, en vue de tirer les leçons de l'expérience et de réajuster si nécessaire les plans d'action ou même certains objectifs (suivi-évaluation), relève également de la réflexion stratégique.

Les méthodes de planification et de management stratégiques ont été développées d'abord dans le monde de l'entreprise ou de l'administration, et sont utilisées aujourd'hui dans de nombreuses catégories d'organisations. Les multiples outils existants ne sont pas tous équivalents quant aux résultats obtenus sur le plan de la dynamique de l'organisation.

Ce chapitre comprend trois parties traitant respectivement du plan stratégique, du plan d'action et du suivi-évaluation. On y trouve les définitions et les principes essentiels qui permettent de raisonner le choix d'une démarche de réflexion stratégique et son application; quelques outils sont également présentés.

\section{La planification et l'élaboration d'un plan stratégique}

\section{II) Les caractéristiques du plan stratégique}

La démarche de planification stratégique permet de s'entendre au sein d'une OP sur les objectifs et les moyens de les atteindre en formulant 
le plan stratégique, généralement conçu sur plusieurs années. Ce document est utile à plusieurs titres :

- il contribue à la cohérence des actions et aide à établir des priorités entre les opportunités et les demandes diverses;

- il permet d'anticiper et de prendre l'initiative vis-à-vis des acteurs extérieurs;

- il facilite la tâche des responsables qui disposent d'éléments pour prendre des décisions à court terme qui répondent aux attentes à plus long terme de leur base.

Le plan stratégique d'une OP doit être assez ambitieux pour motiver les membres tout en restant réaliste afin de ne pas créer d'attentes rapidement déçues qui pourraient entraîner une démobilisation.

Cet équilibre n'est pas toujours facile à trouver. C'est pourquoi la démarche de planification stratégique ne se justifie pas pour toutes les OP, notamment dans le cas d'organisations de petite taille ou très jeunes. Lélaboration trop précoce d'un plan stratégique peut en effet déstabiliser une organisation encore faiblement consolidée.

Un plan stratégique comprend en général plusieurs composantes qui doivent être cohérentes entre elles. Il peut combiner plusieurs entrées : par les filières ou les territoires, ou selon des approches transversales telles que la formation, le crédit, etc.

Un plan stratégique est évolutif. Il doit être révisé périodiquement pour tenir compte des résultats obtenus, de l'évolution du contexte et des transformations de l'organisation elle-même. Mais une OP ne peut pas être continuellement dans une démarche de réflexion stratégique! Sauf événement particulier, un plan stratégique peut raisonnablement être révisé tous les trois à cinq ans.

\section{ID Participation et résultats de la planification stratégique}

Une démarche de planification stratégique n'a de sens que si les membres de l'organisation y participent. Cette démarche constitue d'ailleurs une bonne occasion de renforcer la cohésion de l'organisation. Il est cependant souvent nécessaire à certaines étapes de mobiliser des compétences extérieures pour apporter un appui méthodologique, réaliser des études spécifiques (phase de diagnostic), élaborer des scénarios, rédiger un document.

Lanimation de la démarche est de la responsabilité de l'organisation et est généralement conduite par ses responsables élus, avec l'appui 
de ses techniciens. À défaut de compétences en interne, l'organisation peut faire appel à un animateur extérieur. C'est généralement une personne qui connaît bien l'organisation et qui veille à rester en permanence à l'écoute des responsables.

Dans la planification stratégique, ce n'est pas tellement le document final qui sera important mais plutôt le processus et la démarche adoptés dans la mesure où ils favorisent un changement dans le regard que les membres portent sur leur organisation, dans le sentiment d'appartenance à l'organisation (encadré 4). Ces changements dépendent des mécanismes de communication interne, de la façon dont sont organisés et animés les débats : tous les membres ont-ils pu s'exprimer s'ils le souhaitaient? Cela dépend aussi de la formulation ou la reformulation des objectifs et des résultats à atteindre. La démarche

\section{Encadré 4. Réflexion stratégique à la Fédération des Associations paysannes de Louga au Sénégal (FAPAL)}

La réflexion stratégique de la FAPAL s'est déroulée en plusieurs étapes, étalées dans le temps.

Un premier travail d'analyse de la situation de ses membres a été conduit par les animateurs endogènes et des responsables au début des années 2000, ce qui a débouché sur un plan d'action pluriannuel qui a été ensuite mis en œuvre.

Après quatre ans d'actions, un premier bilan a été dressé. En partant de ce bilan, une rencontre régionale a été organisée en 2007 avec d'autres acteurs du développement et des OP pour réfléchir à l'avenir de l'agriculture et des paysans. Cette rencontre a débouché sur la création de la plateforme de Louga en 2007.

C'est en 2008 qu'a finalement été produit le document d'orientation stratégique proprement dit, qui fixe les orientations à long terme de l'organisation.

Cinq défis stratégiques sont identifiés pour l'organisation :

- viabiliser l'agriculture paysanne;

- promouvoir une économie solidaire et profitable aux ruraux;

- maîtriser les mutations sociales;

- obtenir des politiques adaptées aux intérêts et réalités des paysans;

- adapter l'organisation de la FAPAL.

(d'après Léonard H., 2008; et sur le site http://fapal.wordpress.com) 
de planification stratégique est l'occasion pour les membres de se concerter sur les orientations et de mieux s'approprier l'organisation.

Le plan stratégique constitue aussi une référence pour communiquer et négocier avec l'extérieur, avec les partenaires de l'organisation. Il doit permettre à l'organisation de rester son propre pilote, c'est-à-dire de choisir parmi les opportunités, celles qui sont compatibles avec ses objectifs à long terme et éviter ainsi de se disperser. Il s'agit aussi pour l'organisation de prendre des initiatives, en particulier de rechercher des alliances et des partenariats pour travailler à la réalisation de ses objectifs.

La démarche de planification stratégique est relativement longue. Elle peut prendre de quelques semaines à plusieurs mois mais doit être menée à un rythme assez soutenu pour maintenir la dynamique. Il est préférable qu'elle ne soit pas interrompue par une saison de culture pendant laquelle les producteurs ne sont pas disponibles par exemple. Il est important de ne pas trop s'attarder sur l'étape initiale du diagnostic et de réserver assez de temps pour réfléchir à l'avenir.

La démarche peut s'appliquer à l'ensemble de l'organisation ou être concentrée sur une partie des secteurs ou des territoires où intervient l'OP.

\section{ID Les étapes de la démarche}

Cette démarche peut être plus ou moins formalisée, et organisée de façon diverse. Cependant, elle respecte toujours plus ou moins les différentes étapes proposées par Marie-Rose Mercoiret (schéma 4).

Plusieurs points méritent d'être soulignés :

- la planification stratégique commence toujours par une analyse de la situation actuelle, du chemin parcouru et du contexte dans lequel se situe l'organisation. À cette étape on utilise couramment les outils du diagnostic (voir chapitre 9);

- il importe de ne pas tout sacrifier au moyen ou long terme. La première étape fait souvent ressortir des besoins urgents. Dès lors, l'organisation est amenée à engager en parallèle des actions destinées à répondre à ces urgences tout en poursuivant les activités de planification stratégique proprement dites. De toute façon, il est difficile de dissocier la réflexion stratégique de l'action; on attend rarement 


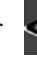

a. connaître

$\leftrightarrow$ « Où en sommes nous ? »

c. définir les orientations

à long terme

$\leftrightarrow$ choisir vers où on veut aller

b. répondre aux urgences

$\leftrightarrow$ Plan d'action pour les priorités

à court terme

\section{d. proposer}

$\leftrightarrow$ élaborer un plan d'action à moyen terme

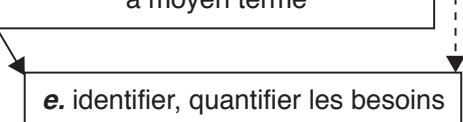

et mobiliser les moyens pour agir, négocier

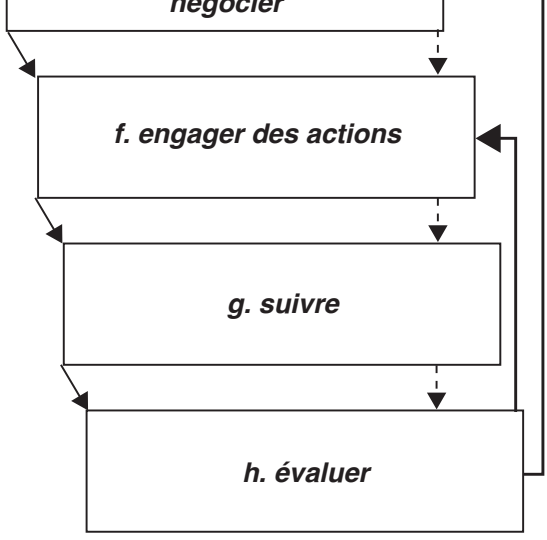

$\longrightarrow$ Démarche de planification stratégique proprement dite

- . - $\rightarrow$ Action dans les domaines prioritaires

$\longrightarrow$ Ajustements résultant du suivi-évaluation

\section{Schéma 4.}

Les étapes de la démarche de planification stratégique (d'après Mercoiret, 1994).

d'avoir défini sa stratégie pour agir et c'est également dans l'action que se précise la vision stratégique;

- la démarche s'engage généralement à l'initiative des leaders et responsables de l'organisation. Elle est cependant d'autant plus utile et 
fructueuse que l'implication des membres est effective. Ce principe est plus ou moins facile à respecter selon les étapes, et selon la taille et la structure de l'organisation;

- les moyens dont on parle ne sont pas seulement logistiques et financiers; il s'agit également des ressources humaines et des compétences (voir chapitre 5). Il est intéressant de ne pas raisonner uniquement en termes de besoins à satisfaire (sous-entendu «avec une aide de l'extérieur») mais de distinguer les actions pouvant être engagées avec les ressources propres de l'OP et celles qui nécessitent la construction de partenariats;

- le suivi et l'évaluation de l'action font partie de la démarche. En effet, l'analyse des difficultés rencontrées pour mettre en œuvre les activités et la comparaison des résultats obtenus par rapport à ce qui était recherché permettent de faire ressortir des besoins non identifiés, ou de préciser les objectifs.

\section{Le plan d'action}

Le plan d'action est la traduction concrète de la réflexion stratégique en termes opérationnels.

Établir un plan d'action, c'est répondre aux questions : qui fait quoi? Quand? Dans quel but? Quel est le résultat attendu? Avec quels moyens?

Un plan d'action est établi à moyen ou court terme (un à deux ans, trois au maximum), car il est nécessaire d'ajuster en permanence la programmation des activités au contexte, qui évolue rapidement.

Une organisation peut établir des plans d'action à différentes échelles, pour ses différents secteurs d'activité. On peut considérer un projet comme un plan d'action particulier qui répond à un objectif spécifique et pour lequel on mobilise des moyens internes à l'organisation, prêtés ou donnés par des partenaires.

Décliner les orientations du plan stratégique en plan d'action ne se fait pas tout seul. Il faut d'abord considérer les différentes voies possibles pour atteindre les objectifs à long terme, évaluer les difficultés ou contraintes, et faire des choix, c'est-à-dire définir des priorités. La construction du plan d'action peut aussi amener à réviser le plan stratégique.

On peut élaborer un plan d'action sans avoir de plan stratégique, dans le cadre de projets notamment. Quand il n'existe pas de plan 
stratégique, cela n'empêche pas de s'entendre sur le cap visé et la vision de l'organisation au moment où on élabore le plan d'action. C'est cette vision qui aide à faire des choix et, quand c'est nécessaire, à trouver les compromis nécessaires pour engager les forces de l'organisation dans une même direction.

En revanche, une démarche de planification stratégique n'a de sens que si elle débouche sur un ou des plans d'action.

La question des moyens disponibles impose souvent de faire des arbitrages : investir ou concentrer les moyens plutôt sur tel ou tel secteur d'activité, par exemple. Ceci relève bien de la stratégie de l'organisation. À un moment donné, une OP peut par exemple privilégier l'équité, avec un volet social, ou plutôt l'efficacité économique pour stabiliser l'organisation ou améliorer sa crédibilité. Très souvent, il s'agit non pas d'abandonner une activité mais de gérer le calendrier de mise en ouvre du plan d'action pour tenir compte des contraintes financières. Lélaboration d'un plan d'affaires ou business plan est alors d'une utilité certaine (encadré 5).

\section{Encadré 5. Plan d'action, plan d'affaires et feuille de route}

\section{Plan d'action}

Il précise la liste des actions prévues pour atteindre un certain but, les moyens humains, matériels et financiers à mobiliser pour les mettre en œuvre, les responsabilités. Un plan d'action se double en général d'un chronogramme qui précise quand on prévoit de réaliser les différentes actions et qui permet de vérifier la cohérence de l'ensemble.

\section{Plan d'affaires (business plan)}

Terme employé dans le domaine économique, notamment par les organismes financiers lors d'attribution de prêts; c'est un plan d'action où on s'attache à préciser les montants et les sources de financement et l'impact économique attendu des actions programmées. Un plan d'affaires peut se rapporter à un projet précis ou correspondre à un plan d'action à moyen terme, dans le cadre d'une OP à vocation économique comme une coopérative par exemple.

\section{Feuille de route}

Contrairement au plan d'action et au plan d'affaires, c'est un outil de très court terme, une feuille de route établit l'enchaînement des actions à entreprendre pour atteindre une étape ou un résultat donné; les personnes responsables et les échéances sont précisées pour chaque action. 
Une fois définies les priorités, on peut opérer de différentes façons pour planifier les actions :

- soit on part des résultats à atteindre pour répondre à la question : quels problèmes résoudre et comment les résoudre ? On indique alors quelles actions permettraient d'aboutir à ces résultats : c'est la planification par objectifs (encadré 6);

- soit on fait des hypothèses sur les différents scénarios, plus ou moins favorables, qui peuvent advenir à partir de la situation actuelle. On détermine ensuite ce qu'on peut faire pour augmenter les chances de mettre en œuvre les bons scénarios, c'est la planification par scénario.

\section{Encadré 6. La méthode de planification des interventions par objectifs (ZOPP)}

La méthode de planification des interventions par objectifs (ZOPP, Zielorientierte Projektplanung) développée par la GTZ (Gesellschaft für Technische ZusammenArbeit) dans les années 1980 est une démarche de planification par objectifs, à laquelle se rattache également l'approche du cadre logique.

C'est une démarche qui se veut participative et qui comprend plusieurs étapes :

- définition de la problématique;

- analyse de la situation incluant l'analyse des parties prenantes (c'est-àdire des individus, groupes ou organisations qui jouent un rôle, direct ou indirect), l'identification des problèmes perçus (arbre à problèmes) et des solutions potentielles (arbre à objectifs), l'analyse du contexte;

- définition des stratégies. On choisit les groupes d'objectifs à mettre en œuvre, en fonction des priorités des bénéficiaires, des compétences et des ressources disponibles, de la faisabilité économique, sociale, de la pérennité, etc.;

- élaboration de la matrice de planification (ou cadre logique) qui précise pour les différents objectifs les résultats attendus, les activités à mener pour $\mathrm{y}$ arriver et les ressources à mobiliser, les risques et les facteurs externes qui peuvent peser sur les activités, les indicateurs qui renseigneront sur l'atteinte des objectifs, le niveau de réalisation et la réussite des activités.

Les outils utilisés, pour le diagnostic notamment, et les méthodes d'animation employées conditionnent largement la qualité de la participation donc la pertinence du produit obtenu.

(d'après Acheroy et Hadjaj-Castro, 2006) 


\section{Le suivi-évaluation}

La réflexion stratégique et la planification, même quand elles sont bien faites, ne garantissent pas que les résultats recherchés seront atteints ou qu'il n'y aura pas d'imprévus. C'est pourquoi le suivi-évaluation est nécessaire.

Le suivi-évaluation est un processus qu'il est souhaitable d'associer à tout plan d'action. Il s'étale tout au long de la mise en œuvre d'une action ou d'un projet.

Le suivi-évaluation comprend trois dimensions (voir tableau 3) :

- l'enregistrement des événements et faits concrets. C'est lui qui permet de savoir précisément ce qui s'est passé et quand. Souvent, on tient une réunion, on réalise une visite ou une mission et on néglige de laisser une trace sous forme de compte-rendu, même bref, des décisions prises et des résolutions;

- l'évaluation par rapport au plan d'action, et éventuellement au plan stratégique. Elle est fondée sur des indicateurs, qualitatifs ou quantitatifs, en principe déterminés quand on a établi le plan d'action. Elle peut servir à réajuster le plan d'action si nécessaire;

- la capitalisation. Cela consiste à tirer des enseignements de l'expérience sur les améliorations à apporter au même type d'action, mais aussi sur le fonctionnement et l'efficacité de l'organisation, sur des pistes d'action nouvelles.

Le suivi-évaluation est d'abord utile à ceux qui le réalisent c'est-à-dire les responsables d'activités, techniciens et élus. Il sert au pilotage des actions. Il peut constituer une très bonne occasion de communication et d'échange au sein de l'OP et, à ce titre, contribue à la réflexion stratégique. Mais le suivi-évaluation est aussi souvent exigé par les bailleurs de fonds comme une composante des projets : il acquiert alors une dimension de contrôle externe qui peut rebuter les membres de l'organisation.

Même quand il n'est pas formalisé, il existe toujours un suivi réalisé par les responsables, sur la base d'échanges d'informations, informels et souvent irréguliers. Certains considèrent que ces pratiques ne permettent pas une capitalisation satisfaisante pour l'organisation dans son ensemble car elles reposent sur des échanges oraux, ce qui ne facilite pas la diffusion objective et large de l'information. En fait, le style de management de certains leaders donne une place importante aux échanges directs dans des réunions régulières. Ces pratiques 
Tableau 3. Les composantes du suivi-évaluation.

\begin{tabular}{|c|c|c|c|}
\hline & Pour qui? Pour quoi? & Par qui? & Comment? \\
\hline Enregistrements & $\begin{array}{l}\text { Pour les membres } \\
\text { et les responsables } \\
\text { de l'OP : garder une } \\
\text { trace des événements, } \\
\text { pouvoir se référer aux } \\
\text { décisions prises }\end{array}$ & $\begin{array}{l}\text { Deux } \\
\text { personnes dont } \\
\text { secrétaire de } \\
\text { séance désigné } \\
\text { en début de } \\
\text { réunion }\end{array}$ & $\begin{array}{l}\text { - Brefs comptes rendus } \\
\text { de réunions : le lieu et } \\
\text { la date, l'ordre du jour, } \\
\text { les personnes présentes, } \\
\text { les décisions prises } \\
\text { et comment (vote ou } \\
\text { autre), éventuellement, } \\
\text { les points de débat } \\
\text { - Comptes rendus } \\
\text { d'activité : Idem } \\
\text { que compte-rendu } \\
\text { de réunion, avec } \\
\text { informations concrètes } \\
\text { sur activité réalisée } \\
\text { (rapport d'activité) }\end{array}$ \\
\hline Évaluation & $\begin{array}{l}\text { Pour les membres } \\
\text { de l'OP et pour les } \\
\text { partenaires (bailleurs } \\
\text { de fonds notamment) : } \\
\text { savoir si on a fait ce } \\
\text { qui était prévu comme } \\
\text { c'était prévu, évaluer } \\
\text { les résultats }\end{array}$ & $\begin{array}{l}\text { Responsables } \\
\text { d'activité avec } \\
\text { les membres } \\
\text { impliqués }\end{array}$ & $\begin{array}{l}\text { En renseignant les } \\
\text { indicateurs choisis en } \\
\text { amont : indicateurs } \\
\text { quantitatifs, indicateurs } \\
\text { qualitatifs, calendrier }\end{array}$ \\
\hline Capitalisation & $\begin{array}{l}\text { Pour l'OP : tirer les } \\
\text { enseignements de } \\
\text { l'expérience, évaluer } \\
\text { la reproductibilité de } \\
\text { l'action, améliorer } \\
\text { son fonctionnement } \\
\text { (efficacité) }\end{array}$ & $\begin{array}{l}\text { Par les } \\
\text { personnes } \\
\text { impliquées } \\
\text { directement } \\
\text { et les } \\
\text { responsables } \\
\text { concernés } \\
\text { avec un appui } \\
\text { extérieur si } \\
\text { nécessaire }\end{array}$ & $\begin{array}{l}\text { Démarche d'analyse } \\
\text { rétrospective : Quels } \\
\text { résultats ont été } \\
\text { obtenus? Attendus ou } \\
\text { inattendus? Comment } \\
\text { s'expliquent-ils? } \\
\text { Quelles ont été les } \\
\text { difficultés? Comment } \\
\text { faire la prochaine fois? }\end{array}$ \\
\hline
\end{tabular}

informelles permettent alors un brassage des idées et des points de vue qui fait vivre l'organisation.

On distingue l'auto-évaluation menée au sein d'une organisation dans le cadre de la réflexion stratégique, et le suivi-évaluation de type projet, plutôt imposé de l'extérieur, qui consisterait à renseigner une grille d'indicateurs dits «objectivement vérifiables», censés rendre compte de l'impact de l'activité considérée. Il n'y a pas de frontière nette entre l'un et l'autre, le suivi-évaluation, comme l'auto-évaluation, sont des outils de management qui peuvent être utiles à l'organisation s'ils sont conçus 


\section{Encadré 7. Le choix des indicateurs}

Indicateur : c'est un «facteur ou variable, de nature quantitative ou qualitative qui constitue un moyen simple et fiable de mesurer ou d'informer des changements liés à l'intervention ou d'aider à apprécier la performance d'un acteur de développement ou la plus-value qu'il apporte».

La construction d'un dispositif d'indicateurs peut introduire des débats d'un grand intérêt si on y associe les bénéficiaires des actions qu'on veut évaluer (les membres d'une OP par exemple); en effet le choix des indicateurs traduit ce qu'on attend concrètement de l'action.

Des indicateurs de qualité doivent répondre à certains critères, ils doivent être notamment :

- spécifiques (un indicateur ne doit mesurer que ce qu'il est censé mesurer par exemple la quantité d'engrais achetée dans un village n'est pas un bon indicateur du fonctionnement du dispositif de crédit intrants car elle dépend aussi de nombreux autres facteurs);

- mesurables (pas forcément quantifiables, mais vérifiables);

- acceptables (par les différentes parties prenantes);

- réalistes (en termes de capacité à le renseigner);

- temporels (compatibles avec le pas de temps considéré).

On peut ou non attacher une valeur cible à un indicateur, par exemple : $70 \%$ des membres ont bénéficié d'un crédit intrants (un indicateur sans cible serait : «opérationnalité du dispositif de crédit : oui ou non»).

Le suivi-évaluation consiste entre autres à renseigner les indicateurs au fur et à mesure du déroulement de l'action (pour savoir si un résultat est atteint et dans quelle mesure) et à ajuster le dispositif d'indicateurs si cela semble pertinent.

(d'après www.cota.be)

et utilisés comme des outils de mobilisation des membres de l'OP. Cette participation des membres est particulièrement utile dès la conception du dispositif et en particulier pour le choix d'indicateurs réellement pertinents et pas trop difficiles à renseigner par la suite (encadré 7).

Parmi les outils existants, la méthode SEPO (matrice des succès, échecs, potentialités, obstacles) est un de ceux qui facilite une analyse à la fois rétrospective et prospective (voir encadré 8).

Un soutien extérieur peut être nécessaire, à la fois sur le plan méthodologique et pour aider à prendre du recul. Cet appui contribue au renforcement de l'OP à condition de ne pas chercher à imposer un 


\section{Encadré 8. La méthode de la matrice SEPO}

La méthode de la matrice SEPO (succès, échecs, potentialités, obstacles) a été développée dans un but d'analyse des entreprises par les employés. Elle consiste à analyser les activités passées puis à regarder vers l'avenir et à remplir une matrice où l'on distingue ce que l'on considère comme des succès ou des échecs (et leurs causes), et ce que l'on perçoit comme des potentialités ou des obstacles pour l'action future :

\begin{tabular}{lll}
\hline activités passées & 1 - succès & 2 - échecs \\
\hline futur & 3 - potentialités & 4 - obstacles \\
\hline
\end{tabular}

Elle peut être mise en œuvre de diverses façons :

- sur le plan de l'organisation : en travaillant individuellement d'abord, ou en constituant des groupes distincts (femmes et hommes, ou éleveurs et agriculteurs) et en comparant ensuite les résultats;

- dans le contenu, en proposant une grille plus fine d'analyse des raisons du succès ou de l'échec, ou en détaillant les différentes dimensions du futur (activités, moyens, partenariats).

Dans tous les cas, il faut prendre le temps de faire la synthèse et de définir des priorités.

NB : SEPO est une variante de l'analyse FFOM (pour Forces, Faiblesses, Opportunités, Menaces; SWOT, en anglais) plus adaptée à l'analyse d'un système, c'est-à-dire l'analyse des différents éléments et des relations entre ces éléments, elle est moins centrée sur les activités.

(d'après www.cota.be)

modèle tout fait et de prendre en compte la dimension de la capitalisation évoquée ci-dessus.

Il faut distinguer l'évaluation (ou suivi-évaluation), qui concerne une action ou un ensemble d'actions, de l'audit, qui évalue les modes de fonctionnement d'une organisation par rapport à des normes. Laudit est réalisé par un expert externe à qui on demande une évaluation sur la base de critères standard. Contrairement au suivi-évaluation, il ne peut être conduit par les acteurs eux-mêmes.

Qu'il s'agisse d'élaborer un plan stratégique, de développer ou de mettre en ouvre des plans d'action, ou de faire fonctionner un dispositif de suivi-évaluation, les questions de la démarche adoptée et de l'accompagnement sont essentielles. Selon les cas, les moyens investis 
peuvent se révéler très utiles à la dynamique de l'organisation ou au contraire rester des exercices formels sans grand impact. Ce sont les méthodes qui favorisent une large implication de la base et l'appropriation des résultats par les membres qui répondent le mieux aux besoins des OP.

En effet, une vision commune partagée par le plus grand nombre, un plan stratégique validé et des plans d'action visibles et régulièrement mis à jour, sont autant d'atouts qui permettent à une OP de mobiliser ses membres dans l'action collective et renforcent les relations au sein de l'organisation (voir chapitre 5). Ils facilitent également la prise de décision des élus et des responsables dans les négociations avec les partenaires (voir partie 3).

\section{Pour aller plus loin}

Mintzberg, H.B. Ahlstrand-Lampel J., 1999. Safari en pays stratégie. L'exploration des grands courants de la pensée stratégique. Pearson Education France, Paris. Coll. Village Mondial. 485 p.

Helming S., Göbel M., 1998. ZOPP. Planification des projets par objectifs. Un guide de planification pour des projets et programmes nouveaux et en cours. GTZ, Eschborn, Allemagne. 34 p.

Site web du Cota : www.cota.be et notamment :

Hadjaj-Castro H., 2007. Fiche 4. Méthode de gestion de cycle de projet. Cota, Bruxelles. 12 p.

Bucci M., Hadjaj-Castro H., 2010. Le suivi des interventions de développement : prise en compte des différentes perspectives des acteurs. Cota hors-série $n^{\circ} 9$. 



\section{$\sqrt{5 .}$ Faire fonctionner une organisation de producteurs}

Comment s'organiser au quotidien pour faire ce qu'on a à faire? La question mérite d'être posée. En effet, la traduction des orientations stratégiques et des principes en actions se heurte souvent aux contraintes quotidiennes, qu'elles soient organisationnelles, humaines, matérielles ou financières. Dans beaucoup de situations, la stratégie d'une OP se définit de façon pragmatique, par un enchaînement de petites décisions prises en réaction à un contexte mouvant, aux menaces et opportunités qu'il offre. Cela n'est pas forcément contradictoire avec le processus logique d'une réflexion et d'une construction stratégique (voir chapitre 4).

Comme le traduit le schéma 5, la problématique du fonctionnement d'une organisation concerne notamment la gestion des ressources. Pour atteindre les objectifs fixés par la réflexion stratégique, divers processus sont à l'œuvre, que nous allons aborder dans ce chapitre. Il s'agit de mobiliser des moyens, de prendre des décisions sur la façon de les employer, de s'organiser pour les valoriser, de rendre des comptes sur leur utilisation, tout cela dans le cadre des textes qui régissent le fonctionnement interne de l'OP.

Sans entrer dans le détail du «comment faire», nous allons rappeler quelques principes ou quelques questions à se poser dans une organisation pour concilier efficacité et bonnes pratiques.

\section{Le cadre : les textes qui régissent l'OP}

Les statuts d'une organisation prennent la forme d'un ou de plusieurs textes qui rappellent les objectifs et fixent à la fois la structure et les règles relatives à son fonctionnement. Les statuts sont établis au moment de la création par les membres fondateurs, mais peuvent évoluer par la suite.

Le plus souvent il existe une législation nationale qui définit les statuts juridiques possibles, les statuts (encadré 9) doivent alors s'y conformer. Pour les OP, il existe en général au moins un statut associatif et un statut coopératif, ou un statut syndical. Le choix d'un statut juridique 


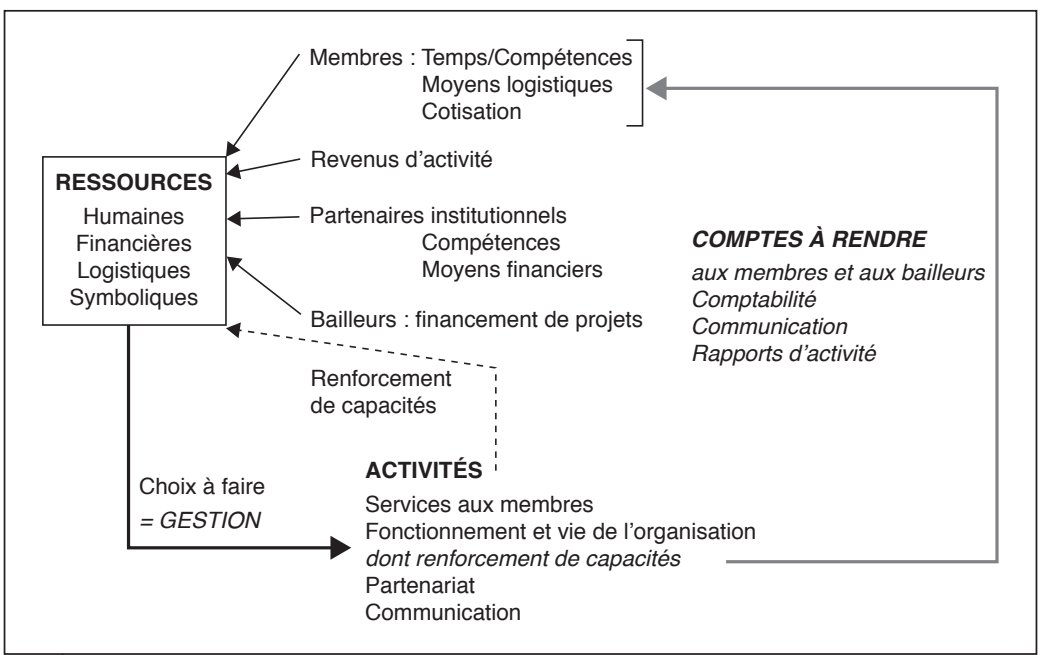

\section{Schéma 5.}

Fonctionnement d'une organisation : la problématique de la gestion des ressources.

implique des obligations légales et des avantages spécifiques, comme par exemple, la défiscalisation des bénéfices pour les coopératives dans de nombreux pays.

Le règlement intérieur fixe plus précisément les droits et les devoirs des membres d'une organisation. Il est généralement établi par les membres en toute liberté et sans obligations juridiques particulières. Il constitue donc le socle de règles que se donne l'organisation en toute connaissance de cause (encadré 9).

Enfin, des cahiers des charges ou manuels de procédures peuvent être définis pour certains aspects ou activités spécifiques. C'est notamment le cas pour les questions financières quand des financements extérieurs importants sont en jeu. Lorsqu'ils sont trop complexes, ces documents de type «manuel de procédure» peuvent alourdir inutilement voire bloquer le fonctionnement d'une organisation. Il est donc important de veiller à leur clarté et à leur simplicité.

\section{La prise de décision et l'exercice du pouvoir}

Les modalités de prise de décision au sein d'une organisation sont définies par les membres au moment de sa création et évoluent au 


\section{Encadré 9. Principaux points relevant des statuts et du règlement intérieur}

Les statuts fixent les principes fondateurs, les objectifs de l'organisation, la vision, les moyens d'action, la nature des ressources, le statut juridique, la structure de l'organisation, le mode de désignation des différents organes, la règle de prise de décision, les moyens de contrôle, les règles concernant la dissolution de l'organisation.

Le règlement intérieur définit qui peut être membre, les droits et devoirs des membres et des salariés, les sanctions éventuelles en cas d'infraction aux règles, les relations entre membres et salariés et l'organisation interne.

cours de son histoire. Elles diffèrent d'une organisation à l'autre ou, au sein d'une organisation, selon les sujets concernés : décisions collectives prises à l'unanimité ou à la majorité, délégation de décision. Ces modalités sont en général définies dans des termes assez généraux par les statuts, car ces derniers sont le plus souvent rédigés très tôt dans la vie de l'organisation, pour lui permettre d'avoir une existence légale. Il n'est pas toujours facile de faire ensuite coïncider les pratiques avec ces textes, parfois insuffisamment réalistes, d'où l'intérêt de les réajuster si besoin (voir ci-dessus).

Différents organes sont impliqués dans la prise de décision : assemblée générale, conseil d'administration, bureau, commissions. Leurs rôles et leurs prérogatives sont fixés par les statuts de l'organisation. Les modalités de prise de décision selon les cas sont prévues dans les statuts ou le règlement intérieur. On parle ici des «grandes » décisions, mais il faut avoir conscience que beaucoup de «petites» décisions doivent être prises également au quotidien, pour lesquelles la façon de faire dépend surtout des responsables et du style de gestion qu'ils adoptent.

La question du pouvoir et de la prise de décision est fortement liée à celle de la représentation au sein de l'organisation, c'est-à-dire aux mécanismes qui amènent à désigner les responsables (élus) de l'organisation aux différents niveaux. Ces mécanismes formels peuvent varier. Ils dépendent en particulier de la structure de l'organisation. Dans une structure pyramidale, chaque niveau désigne les représentants de niveau supérieur : le niveau villageois désigne des responsables pour le niveau régional qui, lui-même en désigne pour le niveau national. On peut également adopter un fonctionnement collégial avec divers 
groupes - producteurs par filière, par zone géographique, salariés qui désignent des membres du conseil d'administration. Les modalités de désignation peuvent également varier : élections, cooptation, etc. Le choix est souvent un compromis entre le souhait d'impliquer la base et l'efficacité6. Il dépend largement de la taille de l'organisation, en nombre de membres, de son aire géographique et des modes de communication en son sein.

Pour comprendre le fonctionnement interne d'une organisation en vue d'améliorer son efficacité, la compréhension des jeux de pouvoir est importante. Le pouvoir au sein des OP s'exprime sous deux formes : un pouvoir formel défini par les statuts qui fixent les règles de pouvoir hiérarchique entre acteurs; des réseaux de pouvoir informel, fondés sur d'autres critères souvent liés à des sphères extra-organisationnelles (âge, groupe, expériences, etc.). La réalité du pouvoir se construit sur un ensemble de critères : capacité à décider, mais aussi statut social, argent, confiance, charisme. Dans les OP, on a souvent une hybridation de différents registres de légitimation du pouvoir.

Il est fréquent de distinguer différents types de leaders et de styles de gestion : gestion autocratique, gestion collégiale, gestion démocratique. Ce qui compte ce n'est pas de porter un jugement moral a priori sur l'une ou l'autre modalité mais d'identifier celles qui sont à l'œuvre, d'en analyser les conséquences pour l'organisation, ses activités, son devenir, etc., et de préciser les formes et les modalités d'appui qui pourraient être mises en œuvre.

\section{La gestion des ressources financières}

Les ressources financières d'une OP peuvent avoir diverses origines : les cotisations des membres individuels ou groupes, les prélèvements sur des ventes de produits ou de services, des ressources négociées avec d'autres acteurs et les pouvoirs publics (taxes parafiscales par exemple), des subventions accordées par des intervenants extérieurs (projets, ONG).

Dans la plupart des cas, ces différentes sources de financement se complètent et c'est d'ailleurs souhaitable pour l'autonomie et la pérennité de l'organisation. Les cotisations des membres représentent en

\footnotetext{
${ }^{6}$ Il ne faut cependant pas opposer de façon simpliste participation et efficacité : une prise de décision très participative peut en effet contribuer à renforcer l'efficacité de l’organisation, en améliorant la mise en œuvre effective des décisions.
} 
général une faible part des ressources de l'organisation mais elles sont essentielles car elles témoignent du sentiment d'appartenance à l'organisation et donnent une image positive de celle-ci. Ces cotisations internes garantissent aussi une indépendance minimale et la possibilité d'assurer la part d'autofinancement de plus en plus souvent exigée par des partenaires extérieurs.

Il ne suffit pas d'avoir des ressources, il faut également les gérer. La gestion financière est un élément essentiel du fonctionnement de l'organisation, qui est souvent à l'origine de tensions. Sans parler des malversations proprement dites, finalement peu fréquentes, plusieurs types de problèmes sont couramment rencontrés : ils mettent en cause le plus souvent la compétence dans la gestion financière, la transparence (information sur l'utilisation des fonds), le sérieux et la fiabilité de la comptabilité. Les tensions sur la gestion proviennent aussi de désaccords sur les choix stratégiques.

On a souvent tendance à confondre comptabilité, simple dispositif d'enregistrement et de traitement des données, et gestion financière qui est une démarche de prise de décision fondée entre autres sur la connaissance des données comptables.

La comptabilité est l'enregistrement des données comptables : à chaque mouvement d'argent ou de produit (sous forme d'argent liquide, de transfert bancaire, de variation de stock ou autre) correspond une écriture.

Les enregistrements peuvent être faits de façon plus ou moins complexe : en partie simple ou en partie double, comptabilité analytique qui permet de ventiler les charges par activité ou non. En général, les modalités plus complexes permettent un meilleur contrôle et une utilisation plus riche. Il existe de nombreux manuels et documents de référence spécifiques ${ }^{7}$ à ce sujet, adaptés aux différentes tailles et types d'organisations.

Le matériel dont on dispose (de l'ordinateur au simple cahier d'écolier) n'a pas beaucoup d'importance. Dans tous les cas ce qui importe c'est la rigueur et la régularité des enregistrements : tout doit être noté, les justificatifs doivent être classés et référencés pour permettre un contrôle ultérieur. En dehors du grand livre où sont portées toutes les écritures, il est souvent utile de tenir, au jour le jour, un cahier de caisse (encadré 10).

\footnotetext{
${ }^{7}$ On peut citer par exemple les outils proposés par Afrique Verte Internationale, téléchargeables sur le site www.afriqueverte.org
} 


\section{Encadré 10. Les enregistrements comptables minimums}

Qu'il s'agisse d'une recette, d'une dépense, ou d'un mouvement d'argent entre différents comptes (de la caisse à la banque par exemple), les enregistrements comptables incluent au minimum pour chaque opération - de façon à pouvoir se rappeler à quoi correspond une écriture - date de l'opération, description de l'opération et motif, montant, référence du justificatif.

Par exemple : le 13/03/2011 / avance à X pour transport réunion fédération du 15 mars / ... F/ Reçu $n^{\circ} \ldots$ plutôt que «13/03 avance de .... F à X».

La gestion financière est une démarche de prévision : elle consiste à analyser un bilan et à élaborer un budget, puis à suivre sa mise en œuvre et, enfin, à interpréter les résultats. Le budget est fondé sur la prévision des ressources et des charges, poste par poste. Lanalyse du bilan permet entre autres de comparer les différentes activités, de s'interroger sur la durabilité de l'organisation.

La transparence de la comptabilité et de la gestion financière est un des éléments clés sur lesquels repose la confiance des membres visà-vis de leur organisation. Différents mécanismes peuvent être mis en place pour assurer cette transparence à travers le contrôle des comptes. Celui-ci peut :

- être réalisé directement par les membres (ensemble des membres ou groupe mandaté). Ceci suppose de publier les comptes sous une forme accessible, et cependant assez précise, et de réaliser un travail explicatif. Lavantage est que cet exercice contribue à renforcer les capacités au sein de l'organisation, mais le travail à produire en amont est important;

- être confié à une expertise extérieure, publique ou privée. La transparence effective repose alors sur le choix de la personne ou de la structure (compétente, indépendante, acceptée par tous). Le rapport produit doit être explicite et utilisable par l'organisation pour la communication interne.

La comptabilité et la gestion peuvent être assurées complètement en interne par des élus ou des salariés de l'organisation ou partiellement externalisées auprès d'un cabinet comptable (encadré 11). Dans tous les cas, il est important que cette fonction soit suffisamment comprise par l'ensemble des élus et représentants pour permettre un contrôle interne réel. Il est par ailleurs souvent utile pour une OP d'avoir 
un commissaire aux comptes, garant extérieur de la régularité des comptes. Ceci est d'ailleurs exigé par certains bailleurs de fonds et spécifié dans certains statuts.

\section{Encadré 11. Répartition des tâches et des responsabilités de gestion}

\section{Comptable}

Technicien en charge de la tenue des comptes et de l'établissement des documents comptables et du bilan, c'est-à-dire de l'enregistrement des opérations, du classement des pièces justificatives, des opérations de clôture. Il applique les règles propres à l'organisation en conformité avec la législation nationale.

\section{Trésorier}

Élu responsable du suivi des opérations financières, c'est-à-dire des recettes, des dépenses, et du contrôle des procédures de décaissement.

\section{Commissaire aux comptes}

Personne extérieure à l'organisation, en général désignée par le conseil d'administration ou l'assemblée générale (selon les statuts), qui vérifie et certifie l'exactitude et la conformité des comptes.

\section{La gestion des ressources humaines}

La gestion des ressources humaines et des compétences est une autre composante importante du fonctionnement des organisations. Elle concerne à la fois les membres de l'organisation - et pas seulement les responsables - et les différents salariés.

Pour la réalisation des activités, les organisations doivent souvent choisir entre internaliser et externaliser les fonctions, en particulier celles qui relèvent de la technostructure et du support logistique (voir chapitre 3). Le choix dépend notamment des ressources et des compétences mobilisables au sein de l'organisation.

Certains membres peuvent disposer des compétences nécessaires mais ce n'est pas toujours le cas. De plus, même s'ils sont compétents, ils n'ont pas toujours la disponibilité nécessaire pour remplir les fonctions voulues. Les organisations sont alors amenées à recruter des salariés pour assurer ces tâches, sous le contrôle des élus. 
Le choix d'un salarié est souvent compliqué car il doit prendre en compte différents aspects du candidat : les compétences, c'est-à-dire sa capacité technique à remplir ses tâches, son système de valeurs (voir chapitre 3 ) et son appartenance sociale qui conditionnent son insertion dans la société locale et dans l'organisation et, enfin, ses exigences en termes de rémunération rapportées aux ressources de l'organisation à court et à moyen termes.

Le processus de recrutement est rendu délicat par les multiples enjeux, parfois contradictoires, auxquels font face les personnes responsables de l'embauche. Il leur faut trouver l'équilibre entre les compétences recherchées et la rémunération envisagée, et elles subissent fréquemment des pressions de la part de membres et de partenaires, en faveur d'un profil ou d'une personne particulière. C'est pourquoi certaines organisations choisissent de confier le recrutement à des cabinets spécialisés externes. Ce choix peut être pertinent à condition que le profil du poste à pourvoir et les critères de choix de la personne aient été clairement définis par les responsables de l'organisation eux-mêmes.

Les relations entre élus paysans et salariés techniciens sont marquées par l'asymétrie : différences de niveau académique, de statut social, de culture ou même d'origine ethnique qui, dans certaines sociétés, pèsent fortement sur les relations. Tout cela peut entraîner des tensions, nées de différences d'appréciation, auxquelles peuvent s'ajouter des problèmes de compréhension mutuelle liés aux formes de langage, au vocabulaire.

Une autre source d'incompréhension, voire de conflits, peut venir du fait que les salariés n'ont que rarement été présents à la naissance de l'organisation et ne comprennent pas toujours, et même ne partagent pas forcément, la vision qui est celle des élus.

Inversement, il peut exister une grande solidarité et une complicité entre responsables élus et salariés; ces liens sont renforcés par le travail quotidien. C'est indéniablement une grande force pour une organisation, mais dans certains cas cela peut contribuer à créer un fossé entre la base et l'équipe dirigeante. Cela peut même aller jusqu'à gêner le jeu démocratique ou affaiblir l'organisation en cas d'alternance. On a vu ainsi des organisations où les nouveaux élus ont licencié des salariés, considérés comme trop proches de l'ancienne équipe. Dans d'autres cas, les salariés eux-mêmes ont choisi de partir; dans ces situations, beaucoup d'informations risquent d'être perdues et le redémarrage de l'organisation peut être difficile. 
Il est essentiel que le partage des rôles entre élus et techniciens soit clair et explicite. Cela suppose une discussion entre les élus et les salariés concernés, dont les conclusions peuvent être portées par écrit et avoir une valeur contractuelle. En particulier, ce document peut alors préciser la répartition des responsabilités et des tâches entre les élus, président et secrétaire, et le coordinateur ou secrétaire général, et entre le trésorier et le comptable.

La quantité et la nature du travail fourni par les uns et les autres, et les avantages qu'ils en retirent, salaires et autres, sont également des points délicats qui ne sont pas toujours traités convenablement. Certains élus sont des employeurs très exigeants, attendant de leurs salariés une disponibilité permanente. Ils ont parfois des demandes et des exigences contradictoires ou déraisonnables, en termes de délais notamment. Ils connaissent souvent mal la législation du travail. À l'inverse, certains salariés jugent sévèrement des élus qui devraient à leurs yeux rester de purs militants, bénévoles et désintéressés, complètement au service de l'organisation, sans tenir compte de la charge que représente leur engagement pour leur exploitation, leur famille.

Il faut reconnaître que le niveau de rémunération d'un salarié, établi sur la base du marché du travail peut être difficile à accepter de la part d'élus qui peuvent avoir des revenus moindres. D'un autre côté, les salariés peuvent avoir l'impression de ne pas être reconnus à leur juste valeur, quand par exemple, ce sont les élus qui ont les honneurs des médias, alors que ce sont eux qui ont écrit les discours et préparé les dossiers.

Ces difficultés se posent presque toujours à un stade ou à un autre de la vie d'une organisation. Lintervention d'un médiateur externe peut alors se révéler utile. Il n'existe pas de solution miracle qui permette d'éviter ces difficultés, mais le fait d'en avoir conscience de part et d'autre contribue déjà à limiter les problèmes. Mieux, la construction d'une culture de l'organisation, salariés et élus étant solidaires, ayant une confiance réciproque, et reconnaissant mutuellement leur complémentarité, facilite le dialogue, même en période de tension. Cela suppose d'investir le temps nécessaire à la communication interne, de s'interroger ensemble, régulièrement, sur le partage des rôles et le fonctionnement de l'organisation. C'est pourquoi, il se révèle ainsi souvent rentable que les salariés soient associés à la réflexion stratégique, tout en prenant soin de les faire participer à des groupes distincts de ceux des membres de l'organisation afin de bien clarifier les différences de points de vue. 
Enfin, quelles que soient les ressources humaines de l'organisation, les compétences disponibles se révèlent presque toujours, à un moment ou à un autre, insuffisantes ou inadaptées. Le recrutement de nouveaux salariés ne peut remplacer la formation des membres, des élus et des salariés en place. Cette question a une importance fondamentale pour les organisations (voir chapitre 4).

Un exemple (encadré 12) illustre bien comment une organisation de producteurs peut instaurer un ensemble cohérent de services pour répondre aux besoins de ses membres, et quelle diversité de ressources humaines elle est amenée à mobiliser pour le faire.

\section{Encadré 12. Ressources mobilisées pour assurer des services aux membres : l'exemple de Mooriben}

Au Niger, les unions membres de Mooriben-Fédération des unions des groupements paysans du Niger (FUGPN) proposent à leurs membres un ensemble de services pour répondre à leurs besoins essentiels. Selon les cas, ces services, supervisés par l'organisation de producteurs, sont mis en œuvre selon des modalités différentes qui font appel à des ressources variées.

\begin{tabular}{ll}
\hline Type de service & Ressources mobilisées et organisation \\
\hline Boutique d'intrants & $\begin{array}{l}\text { Responsables de l'Union } \\
\text { et gérants employés par l'Union }\end{array}$ \\
\hline Banque de céréales & $\begin{array}{l}\text { Responsables locaux avec appui } \\
\text { de l'Union }\end{array}$ \\
\hline Dispositif d'appui-conseil & $\begin{array}{l}\text { Animateurs endogènes (salariés) } \\
\text { et paysans relais bénéficiant } \\
\text { de formations spécifiques }\end{array}$ \\
\hline Radio & $\begin{array}{l}\text { Partenariat avec des radios locales } \\
\text { et formation d'animateurs spécialisés } \\
\text { rattachés à l'Union }\end{array}$ \\
\hline Cellule de communication & $\begin{array}{l}\text { Représentants de l'Union } \\
\text { et personnes ressources }\end{array}$ \\
\hline Caisses mutuelles d'épargne & $\begin{array}{l}\text { Responsables de l'Union avec (ou non) } \\
\text { l'appui opérateur externe. } \\
\text { Initiatives externes positionnées } \\
\text { au sein des Unions }\end{array}$ \\
\hline
\end{tabular}

Warrantage

Financement : Fédération Mooriben, ressources propres + emprunt auprès d'une structure nationale

(d'après Bennegouch et Hassane, 2010) 


\section{La gestion de l'information et la communication}

La communication joue un rôle essentiel dans la construction des relations d'une organisation avec son environnement et avec les autres acteurs. Elle conditionne en partie l'image de l'organisation et c'est pourquoi des moyens conséquents y sont de plus en plus souvent consacrés. L'information venue de l'extérieur concourt par ailleurs au renforcement de compétences des salariés et des élus, et favorise l'innovation.

Mais la gestion de l'information et la communication sont probablement encore plus importantes pour le fonctionnement interne. En effet les flux d'informations sont nécessaires à la coordination entre les différentes composantes de l'organisation; ils permettent de prendre les décisions en temps opportun. C'est un échange à double sens. D'une part, la diffusion d'information sur les activités en cours ou prévues par l'organisation conforte le sentiment d'appartenance des membres, d'autre part, l'information sur la situation, les besoins, les désirs et les aspirations des membres apporte des éléments essentiels pour ajuster le programme de l'organisation.

Par ailleurs, une bonne information des membres se révèle cruciale quand ceux-ci doivent se mobiliser pour appuyer les positions de leur organisation et peser sur les décisions, lors de mouvements sociaux par exemple. Lengagement de chacun repose alors sur une compréhension des enjeux qui ne peut se construire que dans la durée, à partir d'une information régulière.

Il coexiste toujours dans une organisation des circuits d'information formalisés et normalisés, et des circuits informels. Parmi les canaux d'échange formels, on peut citer : lettre d'information ou journal, réunions, rapports, émissions de radio. Certains types de diffusion peuvent même être mentionnés dans les statuts ou dans le règlement intérieur. Les occasions de faire circuler l'information sont multiples : assemblées générales, commissions, groupes de travail, réunions, ateliers. La forme et le contenu des messages que l'on peut faire passer varient selon qu'il s'agit d'échanges individuels, en petits groupes, ou de communication de masse. Si les formes et les contenus sont très variables, il convient toutefois de suivre certaines règles concernant la restitution d'information (encadré 13).

Les échanges informels sont cruciaux également dans le fonctionnement quotidien des organisations, notamment dans les processus d'ajustements, de création et innovation, de résolution de conflit. 
Leur importance est souvent sous-évaluée puisque, par définition, ils laissent peu de traces.

La gestion de l'information suppose un temps de travail et des moyens financiers qui sont souvent sous-estimés. Le coût et les difficultés sont d'autant plus grands que le territoire de l'organisation est vaste ou morcelé et que la structure est complexe, dans le cas d'une fédération par exemple. Les obstacles matériels doivent également être pris en compte, tels que la couverture et la fiabilité des réseaux de téléphonie mobile par exemple.

La conduite des réunions est un élément important de la vie d'une organisation; on n'y attache souvent pas assez d'importance. Quelques règles simples permettent toutefois d'éviter bien des problèmes, qu'il s'agisse de l'animation (encadré 14) ou des conditions matérielles et logistiques (encadré 15).

\section{Encadré 13. Restitution d'informations : les principes}

Le premier principe est que toutes les personnes concernées par une activité doivent être informées des résultats. Par personnes concernées, on entend celles qui ont contribué directement (un producteur qui a participé à une enquête ou à un essai a le droit d'en connaître les conclusions par exemple) ou celles qui ont désigné un représentant (pour participer à une réunion, à une visite) et bien sûr celles dont les responsabilités au sein de l'organisation le justifient. En conséquence, toute étude ou travail de recherche mené dans une organisation doit faire l'objet d'une restitution. Chaque voyage d'étude ou chaque participation à un atelier doit faire l'objet d'un partage d'information avec personnes concernées.

Le deuxième principe est qu'une restitution doit laisser de la place aux réactions des acteurs; elle doit constituer un moteur pour l'action, un outil de mobilisation, à condition d'être à la fois positive et lucide. Elle doit s'attacher à donner une image cohérente des événements de l'organisation (ou du secteur auquel on s'est intéressé spécifiquement), et à mettre du sens là où il n'y en avait pas. Une restitution est l'occasion pour une organisation d'améliorer son positionnement vis-à-vis de l'extérieur.

Le troisième principe est de réfléchir et de préparer des supports pour la restitution d'information en cherchant à s'adapter aux différents publics qui vont $\mathrm{y}$ assister. Les supports visuels sont en effet souvent très utiles, à condition d'être travaillés (pas trop chargés, qu'il s'agisse de transparents ou de diaporama en Power Point) et adaptés au public. Ils peuvent aider à gérer les problèmes de langue (transparents en français et exposé en langue locale par exemple). 


\section{Encadré 14. Organiser et animer une réunion : règles fondamentales}

Une réunion se prépare : dossiers documentés (ce qui ne veut pas dire conclusions préétablies), supports de présentation soignés.

Il doit toujours y avoir une trace écrite d'une réunion : le rôle de secrétaire de séance doit être attribué en début de réunion à quelqu'un qui pourra faire le travail dans un délai acceptable; le choix d'un binôme (élu et salarié) peut être judicieux et donner un résultat plus riche.

La qualité de la participation est d'autant meilleure qu'on a donné aux participants les moyens de se préparer : envoi d'un ordre du jour, informations essentielles communiquées en temps opportun. Il faut prévoir des réunions préparatoires à la base quand certaines décisions stratégiques doivent être prises.

Il vaut mieux prévoir un ordre du jour restreint et laisser le temps aux discussions de se développer. Rien n'est plus frustrant que des débats interrompus, et quand c'est le cas, il faut s'entendre sur les modalités de poursuite de la discussion sous une forme ou une autre.

Il faut savoir sortir des débats stériles : pour cela le président de séance, qui distribue la parole, doit avoir assez d'autorité pour interrompre au besoin certains; il revient à l'animateur de poser les questions ou de présenter les supports visuels qui vont amener les débats sur les points essentiels.

Il est toujours bon de faire une synthèse des conclusions avant de se séparer, de convenir de la date de la prochaine réunion s'il y en a une de prévue.

\section{Encadré 15. Organisation matérielle et logistique des réunions : quelques conseils}

Si on souhaite une participation de qualité, il faut des conditions acceptables notamment en ce qui concerne l'environnement sonore (calme, micro si la salle est grande et résonne).

Les conditions de la participation doivent être clarifiées avant la réunion, au plus tard au début (prise en charge, transport, repas, etc.).

Il convient de respecter les horaires prévus (démarrage et fin) : c'est une des premières règles de gestion des ressources humaines que d'éviter de gaspiller le temps de ceux qui sont à l'heure pour attendre ceux qui sont en retard, quelle que soit leur position. 


\section{Légitimité et représentativité : les enjeux du fonctionnement pour les organisations}

Le fonctionnement d'une organisation conditionne non seulement sa crédibilité interne mais également ses relations avec l'extérieur et notamment l'image qu'elle donne. En particulier la légitimité et la représentativité de ceux qui représentent l'OP sont très souvent évaluées, à tort ou à raison, sur le fonctionnement démocratique de l'organisation. La légitimité s'ancre par ailleurs dans l'action, dans les services rendus effectivement aux membres et à l'organisation, c'est une valeur interne à l'OP en quelque sorte. Lévaluation de la représentativité est souvent fondée sur la structure, sur les modes de désignation des élus et sur le respect des textes de l'organisation. La question de la représentativité est souvent posée par les acteurs extérieurs à l'OP : «Telle ou telle organisation et tel ou tel leader sont-ils représentatifs? », en sous-entendu "avons-nous de bonnes raisons de l'écouter, de répondre à ses requêtes?».

Les organisations font face à différents défis en matière de structure et de fonctionnement.

- Elles doivent s'adapter à des cadres juridiques et normatifs toujours plus complexes. De plus en plus, elles doivent respecter des règles de fonctionnement contraignantes imposées par la loi ou par les partenaires extérieurs. Cela peut les obliger à recourir à des expertises techniques pas toujours faciles à identifier, ni à financer.

- Dans leur fonctionnement et leurs orientations, elles ont à gérer les tensions entre efficacité et inclusion. Pour obtenir le maximum de résultats, une OP peut ainsi être conduite à travailler plus particulièrement avec certains de ses membres, ce qui implique de fait une certaine exclusion des autres. Au contraire, la recherche d'un développement harmonieux, pour l'ensemble des membres quelle que soit leur situation, qui répond à un objectif d'inclusion, risque de compromettre l'efficacité de l'OP. C'est le cas par exemple d'une OP qui investit plus pour fournir des services aux membres les plus démunis ou marginaux. Ce type de tension s'observe couramment dans les organisations engagées dans la commercialisation de produits vers des acheteurs exigeants sur le plan des normes de qualité.

- Il existe également des tensions entre formalisme et souplesse. La formalisation des règles de fonctionnement est nécessaire et elle est de plus en plus exigée par les partenaires. Cette mise aux normes peut d'ailleurs être l'occasion de débats fructueux entre les différents

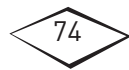


membres des groupes. Néanmoins, le formalisme peut conduire à des rigidités qui limitent la spontanéité des relations avec et entre membres et rend l'organisation plus distante, ce qui peut s'avérer préjudiciable pour l'OP.

- Enfin toutes les OP doivent gérer leurs relations avec leurs membres et notamment les conflits entre les attentes de ces derniers et les capacités des organisations, la transparence de l'information, la participation à la décision, la définition des régimes d'encouragement et de sanctions.

En conclusion, il n'y a pas de mode de fonctionnement parfait. Les modalités doivent être réfléchies en fonction de la mission que s'est fixée l'organisation, des contingences (ressources humaines et financières entre autres) et du contexte de la société dans laquelle elle s'insère. Le mode de fonctionnement n'est donc pas établi une fois pour toutes : les règles internes doivent être revues régulièrement pour être adaptées à la réalité.

Lensemble des questions abordées ci-dessus conditionne en fait la durabilité de l'organisation. Or tout ne marche pas toujours comme il conviendrait, pour des raisons liées à l'environnement mais aussi par manque de certaines compétences; c'est à cet état de fait que tentent de remédier les actions de renforcement de capacités qui vont être étudiées dans le chapitre 6. 



\section{$\sqrt{6 .}$ Renforcer les capacités des organisations de producteurs}

Une des clefs de l'autonomie des OP c'est d'avoir la maîtrise des compétences qui leur sont directement nécessaires. Leur crédibilité (vis-à-vis de l'extérieur) et la légitimité de leurs responsables (vis-à-vis des membres) reposent sur leur capacité : - à faire fonctionner l'organisation en conformité avec le code de bonnes pratiques, ce qui ne signifie pas suivre un modèle standard (chapitres 4 et 5);

- à prendre en compte les besoins des membres et à y répondre par des services de qualité (chapitre 7);

- à mener, avec ces membres, des activités profitables (chapitre 7);

- et à assumer les responsabilités et jouer les rôles qu'on attend d'elles (chapitre 2) à l'interface avec d'autres acteurs publics ou privés (voir partie 3).

Or il est rare que toutes les compétences soient présentes au sein d'une organisation. Les OP sont donc amenées à s'engager dans des actions de renforcement de capacités qui constituent un volet important et délicat de leurs activités. Il n'est pas toujours indispensable, ni même pertinent de disposer de toutes les compétences en interne. C'est pourquoi la question doit être abordée sous plusieurs angles et aboutir à un plan de formation global, pour lequel il convient de chercher des moyens adéquats.

Nous donnerons dans ce chapitre un aperçu des principales questions à se poser et des principes à respecter pour que les activités de formation contribuent réellement à renforcer l'organisation.

\section{Le renforcement de capacités ou la formation}

La distinction entre renforcement de capacités et formation pourrait faire l'objet de longs discours. Pour rester simple, on peut dire que le renforcement de capacités est le résultat attendu des actions de formation, mais qu'il peut également être obtenu par d'autres moyens, comme le résume le tableau 4. 


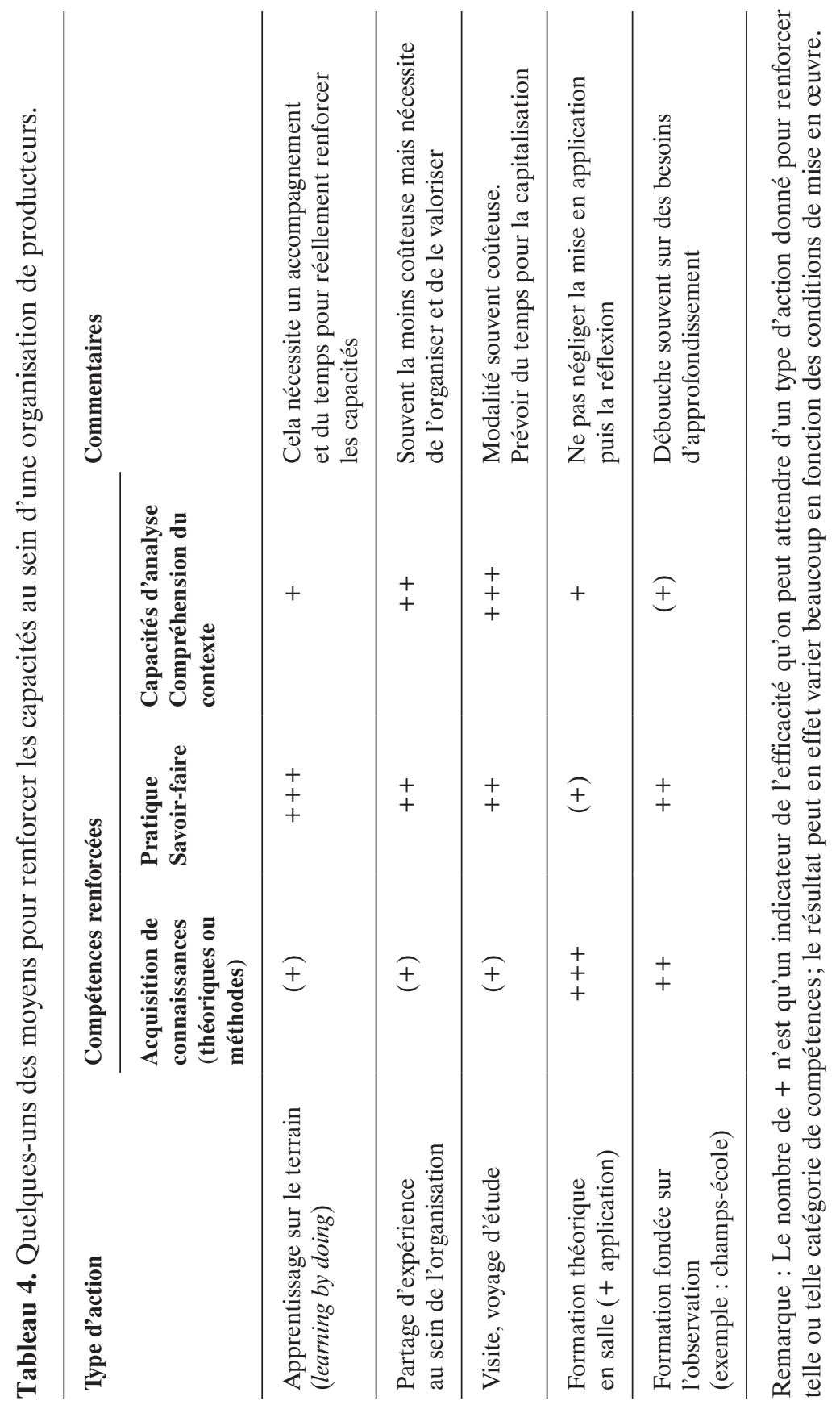




\section{Les objectifs des formations}

Pour une OP, les activités de formation répondent à deux grands types d'objectifs complémentaires : répondre à des besoins exprimés par les membres et renforcer l'organisation.

Dans le cadre des services rendus à ses membres, une OP peut viser à : - améliorer leurs performances techniques par la formation à des techniques nouvelles;

- développer leurs capacités en gestion pour les aider à améliorer la rentabilité de leur activité par la formation aux outils de calcul économique;

- leur faciliter tout simplement l'accès à l'information et les aider à la maîtrise de leur environnement. C'est par exemple la raison d'être des programmes d'alphabétisation qui, au-delà du bénéfice individuel pour les membres, contribuent à améliorer le fonctionnement et la communication au sein de l'organisation et à faciliter les actions entre autres de conseil (encadré 16).

\section{Encadré 16. Étude de cas : la Fédération des Paysans du Fouta Djallon (Guinée)}

La Fédération des Paysans du Fouta Djallon (FPFD) inclut dans son programme d'activités des actions d'alphabétisation au profit de ses membres, et notamment à destination des femmes. En effet, l'analphabétisme est un obstacle à la représentation des femmes dans les instances dirigeantes en proportion du nombre de membres des groupements. Après avoir privilégié l'alphabétisation fonctionnelle, la fédération a développé également des cours de français, puisque les documents préparatoires aux réunions sont en général dans cette langue.

Dans d'autres cas, l'objectif de la formation est le renforcement institutionnel de l'organisation à travers l'acquisition, le renforcement ou l'actualisation des compétences nécessaires à la conduite des différentes activités de l'OP, qu'il s'agisse des services aux membres ou de la représentation des intérêts. Ceci répond à un besoin d'efficacité, et a également un rôle stratégique.

- Même quand elle participe officiellement à des instances de concertation ou de décision, une OP risque de n'être pas considérée comme un partenaire à part entière par ses interlocuteurs du secteur public ou privé. La formation des représentants les rend plus crédibles et renforce leur position dans les négociations. 
- La formation des leaders, en renforçant leurs capacités d'analyse pour saisir les enjeux sociopolitiques, contribue à consolider l'identité de leur organisation. Elle leur permet d'animer la réflexion interne.

- Le fonctionnement de l'organisation met en jeu des activités qui mobilisent des compétences en animation, en communication et en gestion. Certains membres ont eu l'occasion d'acquérir ces compétences par ailleurs, par exemple dans des mouvements de jeunesse, mais pas toujours. La formation peut pallier ces carences.

- Enfin la formation des élus est importante aussi en interne, notamment pour leur permettre de contrôler la technostructure, notamment sur le plan de la gestion financière mais pas seulement.

\section{L'élaboration d'un plan de formation}

Une fois définis les objectifs assignés à la formation, il s'agit de concevoir un dispositif qui permette d'y répondre : c'est ce qu'on nomme l'ingénierie de formation, on parle ici de plan de formation. Dans le cas d'une OP, il est en effet très rare qu'un objectif de renforcement de capacités puisse être atteint par une action ponctuelle isolée; il faut donc raisonner en termes de plan de formation. Ce plan met en jeu des actions de différentes natures, réparties dans le temps et dans l'espace, s'adressant à différents publics et articulées entre elles.

Pour concevoir un plan de formation, il s'agit de répondre aux questions : pourquoi former ou former à quoi? Qui former? Comment former? Qui doit concevoir et mettre en ouvre une formation pour les OP?

\section{ID Pourquoi une formation?}

C'est en général lors d'une réflexion stratégique ou du développement d'un plan d'action (voir chapitre 4) ou encore à l'occasion d'une démarche de diagnostic (voir chapitre 9) que sont mis en évidence des déficits de compétences au sein de l'OP. Ces manques peuvent concerner :

- le domaine managérial et la vie de l'organisation, aux différents niveaux;

- des connaissances et des savoir-faire techniques;

- la compréhension du contexte dans lequel évolue l'organisation et des relations avec les partenaires. 


\section{Qui former?}

C'est bien sûr le «pourquoi? » qui permet de définir le groupe-cible ou la catégorie de personnes à qui s'adresse l'activité de formation.

Cette question reste souvent délicate pour les organisations. En effet, l'accès à la formation étant perçu comme un avantage, on peut avoir tendance à répartir ce bénéfice entre le maximum de membres. Mais cette option est peu compatible avec des parcours de formation étalés dans le temps.

Un large renforcement des capacités stratégiques est également un enjeu majeur du jeu démocratique. Si trop peu de personnes sont compétentes au sein de l'OP, la marge de manœuvre pour la répartition des postes de responsabilité est restreinte, et la capacité de contrôle et de dialogue entre les élus et leur base, ou avec les techniciens, est limitée.

C'est à ces difficultés que répondent les dispositifs de formation en cohortes où plusieurs groupes vont successivement suivre le même parcours. Il est important également que les formations stratégiques ne concernent pas seulement un tout petit groupe de leaders en place.

Lidentification précise des participants à une action de formation repose sur l'application de critères de choix, définis de façon aussi transparente que possible, en tenant compte de l'organisation de la formation et des besoins auxquels elle répond.

\section{ID Comment organiser la formation?}

La réponse à cette question dépend des points précédents, et comprend plusieurs aspects : formation ponctuelle ou en plusieurs étapes, méthodes pédagogiques, outils d'apprentissage. La question de l'articulation entre l'expérience de terrain et l'acquisition de connaissances se pose toujours. En particulier quand les formations portent sur des savoir-faire ou des outils, il est nécessaire de revenir sur la mise en pratique de ces outils et de susciter une analyse critique. C'est pourquoi, dans le cadre de dispositifs de formation en plusieurs modules espacés dans le temps, on demande souvent aux stagiaires de faire un travail de terrain, ou de mener une recherche, seuls ou en groupes, dans l'intervalle entre deux modules. Cela est très utile à condition de prévoir un temps suffisant de restitution et de réflexion partagée sur les enseignements qu'on peut tirer de l'expérience. De même, on néglige souvent le co-apprentissage, souvent très enrichissant, entre membres 
du groupe : or, pour que les gens apprennent les uns des autres, il faut prévoir du temps pour les échanges.

Une formation ne se limite pas forcément à des contenus théoriques transmis en salle. Les voyages d'échanges, les visites, certaines réunions contribuent également au renforcement des capacités de ceux qui y participent. Pour qu'elles jouent pleinement leur rôle ces actions ne doivent cependant pas être isolées, un temps de capitalisation est nécessaire pour dégager les leçons à tirer et consolider les nouvelles connaissances éventuelles (voir schéma 6 et encadré 17).

\begin{tabular}{|c|c|c|c|}
\hline \multicolumn{2}{|c|}{$\begin{array}{l}\text { Renforcement } \\
\text { des capacités }\end{array}$} & Objet & Impact pour l'OP \\
\hline \multirow[t]{3}{*}{$\begin{array}{l}\text { Services } \\
\text { directs } \\
\text { aux } \\
\text { membres }\end{array}$} & Membres & Gestion $\longrightarrow$ & $\begin{array}{l}\text { Meilleurs résultats } \\
\text { techniques } \\
\text { et économiques } \\
\text { Idem + communication } \\
\text { plus facile (vie démocratique) }\end{array}$ \\
\hline & $\begin{array}{c}\text { Spécialistes } \\
\text { (salariés et élus) }\end{array}$ & $\begin{array}{l}\text { Champs d'activité } \longrightarrow \\
\text { de l'OP }\end{array}$ & Plus d'efficacité \\
\hline & Représentants (élus) & Rôle d'interface $\longrightarrow$ & Plus efficace et plus crédible \\
\hline \multirow{2}{*}{$\begin{array}{l}\text { Être plus } \\
\text { efficace }\end{array}$} & Animateurs, leaders & Réflexion stratégique $\rightarrow$ & Vision plus claire \\
\hline & $\begin{array}{c}\text { Élus aux différents niveaux } \\
\text { Tous élus et membres }\end{array}$ & Communication $\longrightarrow$ & $\begin{array}{l}\text { Pépinière de leaders } \\
+ \text { vie démocratique }\end{array}$ \\
\hline
\end{tabular}

\section{Schéma 6.}

Objectifs et impacts des différentes actions de formation.

\section{II) Qui doit concevoir et mettre en œuvre un plan de formation?}

La conception d'un plan de formation relève de la réflexion stratégique. Quand il s'agit d'une formation ponctuelle, elle s'inscrit dans un plan d'action à moyen terme ou un projet. Dans tous les cas, c'est donc l'OP qui est seule habilitée à faire ce travail, avec l'appui éventuel d'alliés disposant de compétences spécifiques en ingénierie de formation ou ayant été associés à la réflexion stratégique.

S'agissant du contenu du plan de formation et des thèmes à traiter il est évidemment préférable de partir d'une analyse précise des besoins pour établir les termes de référence de la formation plutôt que de 


\section{Moyens humains : internes ou externes?}

La question de se doter de compétences internes en formation se pose un jour ou l'autre à une organisation et dans de nombreux cas, il existe un ou plusieurs salariés chargés de la formation, notamment au sein des fédérations (encadré 18). L'avantage est d'avoir une meilleure maîtrise tant sur le plan de l'organisation pratique (calendrier) que des coûts, l'inconvénient est que ces personnels ne sont pas spécialistes de tout et qu'il faut donc savoir ne pas tout leur demander. En revanche, ces compétences internes sont essentielles pour la définition des termes de référence, le suivi et l'évaluation des formations.

On peut citer quelques critères importants pour choisir un formateur ou un animateur : expérience, qualités pédagogiques, connaissance spécialisée du sujet, maîtrise de la langue locale, mais également connaissance du contexte et coût de la prestation. La capacité à mettre en ouvre des démarches de formations participatives ou interactives, ancrées dans l'expérience des participants est essentielle. Il est difficile de trouver la structure ou la personne qui présente tous les caractères recherchés, ce qui impose de définir des priorités en fonction notamment de l'objectif et du public concerné.

Pour lever tout ou partie de ces contraintes, la formation peut être mutualisée entre plusieurs organisations. Cette mutualisation peut être ponctuelle ou régulière et peut prendre différentes formes. Une autre option est la création par une ou plusieurs OP de centres de formation qui offrent à la fois les infrastructures et abritent une équipe de formateurs.

\section{Encadré 18. Étude de cas : le Centre interprofessionnel pour la formation au métier de l'agriculture (CIFA)}

Pour répondre aux besoins dans leur zone, des OP de la région de SaintLouis au Sénégal ont participé à la création d'une association, le Centre Interprofessionnel pour la Formation aux métiers de l'Agriculture (CIFA), qui regroupe des OP et d'autres acteurs du développement, publics et privés. Le CIFA est doté d'infrastructures (salles et hébergement) et abrite une équipe de formateurs susceptibles de répondre à diverses demandes.

Il développe sa propre offre de formation, répond à des demandes spécifiques émanant d'OP ou d'autres acteurs et accueille des réunions, des séminaires et des formations organisés par les OP sénégalaises (de la région et au-delà), des collectivités locales, etc. 


\section{Ressources financières}

Quelle que soit la tendance à la privatisation des services et la pression exercée par certains partenaires dans ce sens, les producteurs, que ce soit à titre individuel ou leurs organisations, n'ont en général pas les moyens de financer intégralement les formations. Conscients de ce point, de nombreux bailleurs de fonds acceptent de financer des actions de renforcement de capacités. Cependant, tous n'acceptent pas que l'OP soit son propre maître d'ouvrage, c'est-à-dire qu'elle organise et gère elle-même la formation, en conformité avec le plan de formation accepté. Cette position répond à un souci de qualité des formateurs mais a au moins deux inconvénients : les délais sont souvent très longs entre l'émergence d'un besoin et l'action de renforcement de capacités proprement dite; l'OP n'est pas en position de discuter avec le formateur ni de peser pour améliorer une prestation qui ne serait pas satisfaisante.

\section{Le cas particulier de la formation initiale au métier d'agriculteur}

Dans de nombreux pays, les dispositifs de formation au métier d'agriculteur qui existaient par le passé ont périclité avec les plans d'ajustements structurels. Or dans le même temps, on a enregistré une rapide évolution du contexte de l'agriculture : dégradation des conditions de production, sur le plan du climat et de la fertilité des sols notamment, écart croissant entre agriculture vivrière qui, souvent, ne permet plus l'autosubsistance et agriculture marchande voire commerciale, de plus en plus exigeante vis-à-vis de la qualité. Ceci impose aux producteurs pour survivre de trouver leur place dans des filières existantes ou de créer de nouvelles filières afin de mettre sur le marché des produits qui répondent à une demande de plus en plus exigeante, de contrôler les coûts de production, de négocier avec les autres acteurs des filières, etc. Le déficit de formation initiale est donc extrêmement pénalisant à la fois pour les agriculteurs et leurs familles, pour les dynamiques de développement local, voire pour les économies nationales. Il est de la responsabilité des OP de s'impliquer dans les processus de réformes de la formation professionnelle agricole qui sont une des dimensions de la politique agricole quand ils existent ou de revendiquer la réactivation de ces dispositifs en cohérence avec leur projet pour l'agriculture. 


\section{L'impact du renforcement des capacités}

Le schéma 6 (voir p. 82) fait ressortir notamment pourquoi le renforcement des capacités est un enjeu majeur pour la majorité des OP. Il montre également que non seulement la définition des thèmes de formation mais également le choix des personnes formées sont déterminants de l'impact de ces activités pour l'organisation.

Soulignons d'ailleurs ici que la formation ne permet pas de résoudre tous les problèmes; elle peut même, en creusant l'écart entre ceux qui savent et les autres, contribuer aux dérives et aux dysfonctionnements. Par ailleurs, toutes les formations ne renforcent pas l'autonomie et l'efficacité des OP et de leurs membres; certaines actions de formation, en diffusant des idées ou des modèles figés, contribuent à freiner l'imagination. C'est pourquoi le mode de formation et le choix des formateurs sont essentiels.

\section{Pour aller plus loin}

www.reseau-far.com 


\section{$2 \mathbb{N}|0\rangle \| \mathbb{P}$ Q}

\section{Renforcer les organisations de producteurs dans leurs relations avec leur environnement}

La plupart des OP ne se contentent pas de répondre à des besoins de leurs membres par le biais de services internes. À un moment ou un autre de leur histoire, elles sont amenées à travailler avec l'extérieur dans le cadre de partenariats plus ou moins formalisés. Cette fonction d'interface peut même avoir motivé la création d'une OP; elle repose sur des principes et prend des formes qui varient en fonction de l'objet du partenariat (à visée technique, ou plutôt politique) et des partenaires eux-mêmes. Dans cette troisième partie, nous proposons donc quelques repères pour l'analyse des relations entre les organisations de producteurs et leur environnement. Nous distinguons deux grands registres d'analyse : les enjeux de la coordination et de la construction de partenariats, principalement dans les champs technique et économique (chapitre 7) puis la participation des OP à l'élaboration des politiques agricoles et développement rural (chapitre 8). Dans tous les cas, un des enjeux majeurs pour l'OP est de se positionner dans une relation équilibrée. Cela suppose que l'ensemble des parties ait une vision juste des forces et des faiblesses de l'organisation, ce qui repose sur une démarche de diagnostic, qui fait l'objet du chapitre 9. 



\section{$\sqrt{7 .}$ Les partenariats à visée technique}

Il importe de répondre à cette question pour deux raisons principales. D'une part, les OP sont des acteurs de la société civile et, dans beaucoup de cas, des opérateurs économiques. En tant qu'acteur collectif, elles cherchent à faire ce que chaque membre individuellement ne pourrait pas faire ou pas aussi bien. Dans ce cadre, elles sont nécessairement en interaction 71 avec leur environnement. D'autre part, elles sont fréquem-
ment sollicitées par les autres groupes d'acteurs, à la recherche d'interlocuteurs ou de partenaires légitimes et efficaces.

Or, les processus suivis et les difficultés rencontrées varient avec l'objet du partenariat (accès au marché, financement, etc.).

De plus, de multiples acteurs sont en relation avec les OP. On peut les répartir globalement en trois grands types avec lesquels les rapports à construire sont de nature différente : les acteurs privés (marchands, commerçants, transformateurs, consommateurs), les organismes d'appui (bailleurs de fonds, ONG locales ou internationales, experts), et les pouvoirs publics (services centralisés ou décentralisés de l'État, administrations publiques, élus locaux ou nationaux) (Mercoiret, 1994).

Par ailleurs, dans le contexte de désengagement des États, on constate fréquemment une carence dans la coordination des interventions des divers acteurs qui assurent tout ou partie des fonctions d'appui à l'agriculture. Les OP peuvent avoir à jouer ce rôle de coordination pour assurer une cohérence des interventions, dans l'intérêt de leurs membres.

Dans ce chapitre, nous aborderons donc les grandes fonctions que peuvent assurer les OP au service de leurs membres à l'interface avec d'autres acteurs pour faciliter l'accès aux marchés, aux financements de l'agriculture, à l'innovation technique et la gestion des biens partagés. Dans chacun de ces domaines, nous verrons quelles activités permettent de répondre aux principaux enjeux. Nous conclurons en proposant quelques éléments de réflexion transversaux aidant à la construction des partenariats équilibrés et durables. 


\section{L'accès des producteurs aux marchés: le rôle des OP dans la coordination et la gestion des filières}

Au cours des deux dernières décennies, l'accès des producteurs aux marchés est devenu à la fois essentiel (une part de plus en plus grande des productions est commercialisée) et plus difficile comme le reconnaît la Banque mondiale (voir encadré 19). En effet, alors que dans les années 1980 la fonction de commercialisation était encore couramment assurée par l'État et que les marchés étaient fortement régulés, les décennies 1990 et 2000 ont été marquées par plusieurs évolutions : - le renforcement des macro-acteurs ${ }^{8}$ au sein des filières, dans les processus de transformation et de commerce, jusque dans la distribution; - l'émergence de nouvelles demandes des consommateurs en termes de caractéristiques des produits et de formes de production, en particulier sur la sécurité sanitaire, l'impact environnemental. Ces exigences se sont traduites par le développement des normes publiques et privées entraînant un renforcement des contraintes pour les producteurs;

- une libéralisation du commerce international (réductions des barrières douanières, accord de libre commerce), qui, accompagnée d'une réduction des coûts des transports internationaux, a entraîné une concurrence accrue entre zones de production, même très éloignées.

Face au retrait des États des fonctions marchandes et aux demandes fortes des producteurs ruraux pour que soient réglés les problèmes d'accès aux marchés, de nouvelles OP se sont constituées et des OP existantes se sont positionnées pour faire face à ces enjeux.

Le schéma 7 résume les principales difficultés que rencontrent les producteurs, et fait ressortir les atouts de l'action collective :

- réaliser des économies d'échelles (achat ou vente groupés, transformation en gros);

- augmenter le pouvoir de négociation avec les autres acteurs de la filière (prix, régularité);

- sécuriser l'accès aux marchés (contrat de moyen terme, etc.);

- réduire ou mutualiser les coûts de transaction (en mutualisant la recherche de partenaires commerciaux, la recherche d'information, le suivi des contrats, etc.).

\footnotetext{
${ }^{8}$ Entreprises de dimension nationale, internationale, voire mondiale, souvent en situation de monopole ou d'oligopole.
} 


\section{Encadré 19. Les difficultés des petits producteurs pour l'accès au marché}

Les transformations des marchés locaux, régionaux et mondiaux ont généré une série d'opportunités pour les petits producteurs mais leur aptitude à profiter de ces opportunités peut être fortement compromise par :

- un manque de capitaux, d'actifs, de compétences et d'information pour être compétitifs sur des marchés gouvernés par les acheteurs;

- des coûts de transaction élevés liés à la petite taille de leur entreprise ;

- un accès limité à des services abordables et fiables dont ils ont besoin pour augmenter la productivité et améliorer la qualité;

- un pouvoir de négociation faible sur les marchés locaux et internationaux contrôlés par les acheteurs;

- une influence limitée sur les politiques locales nationales et internationales et sur les pratiques de gouvernance qui affectent les marchés.

Pour surmonter ces défis, les petits producteurs doivent renforcer leurs capacités à soutenir la concurrence sur les marchés, améliorer leur accès aux ressources externes, et augmenter leur pouvoir de négociation et leur influence.

(d'après Banque mondiale, 2007)

Les initiatives des OP pour répondre à ces difficultés sont multiples, dans leur ampleur et dans leur forme. Pour atteindre ces résultats différentes stratégies sont possibles; elles mettent en jeu trois grands registres d'action qui peuvent coexister, présentés dans le tableau 5.

Les formes de partenariats dans les filières sont multiples et peuvent se faire selon deux grandes directions :

- partenariats entre OP ou coordination horizontale (pour les mêmes fonctions d'une filière), par exemple fédérations de commercialisation ou plateformes de commercialisation entre plusieurs OP, etc. (encadré 20);

- partenariats entre OP et entreprises privées au sein des filières ou coordination verticale. Cette forme de partenariat peut être nouée avec des entreprises en amont (approvisionnement en intrants) ou en aval (commercialisation ou transformation) de la production. Elle peut prendre la forme de contrats plus ou moins formels, de plus ou moins longue durée, incluant ou non des prestations de services liées (avance de financement, conseil technique). Ce type de partenariat peut conduire à maintenir des asymétries de pouvoir entre acteurs des filières et des formes de dépendances entre producteurs et autres 


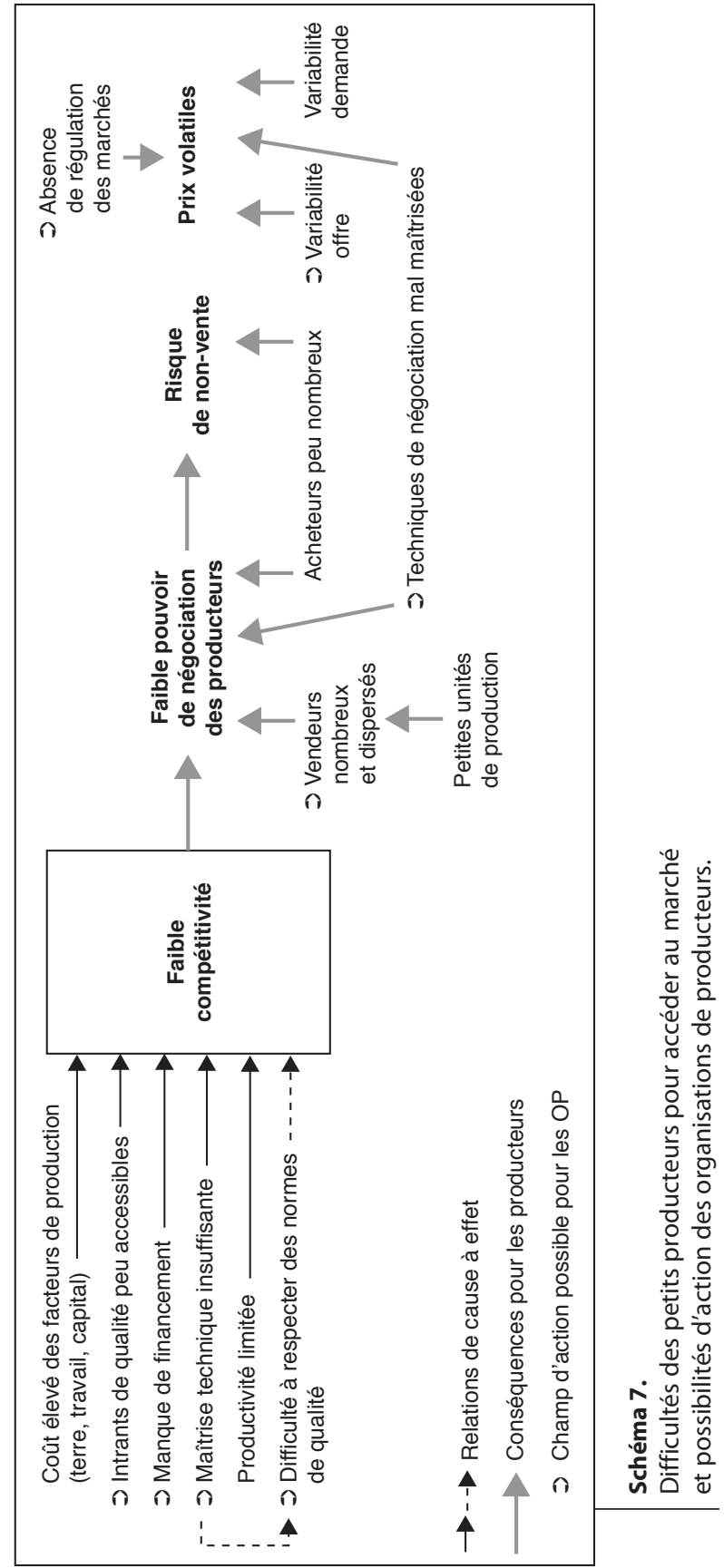


Tableau 5. Exemples d'actions que peuvent mener les OP pour faciliter l'accès au marché.

\begin{tabular}{|c|c|c|c|}
\hline $\begin{array}{l}\text { Registre } \\
\text { d'action }\end{array}$ & $\begin{array}{l}\text { Améliorer } \\
\text { la compétitivité } \\
\text { sur les prix }\end{array}$ & $\begin{array}{l}\text { Améliorer la qualité } \\
\text { et l'image } \\
\text { des produits }\end{array}$ & $\begin{array}{l}\text { Renforcer } \\
\text { le pouvoir } \\
\text { de négociation }\end{array}$ \\
\hline \multirow[t]{3}{*}{$\begin{array}{l}\text { Services directs } \\
\text { aux membres }\end{array}$} & $\begin{array}{l}\text { Fourniture } \\
\text { d'intrants }\end{array}$ & \multirow{3}{*}{$\begin{array}{l}\text { Formation } \\
\text { aux normes, } \\
\text { à la qualité, au } \\
\text { développement } \\
\text { de marques, à la } \\
\text { certification }\end{array}$} & \multirow{3}{*}{$\begin{array}{l}\text { Vente groupée } \\
\text { de produits, } \\
\text { achat groupé } \\
\text { d'intrants }\end{array}$} \\
\hline & $\begin{array}{l}\text { Formation } \\
\text { technique }\end{array}$ & & \\
\hline & $\begin{array}{l}\text { Transformation } \\
\text { des produits }\end{array}$ & & \\
\hline \multirow{4}{*}{$\begin{array}{l}\text { Intermédiation } \\
\text { Facilitation }\end{array}$} & Accès aux intrants & \multirow{4}{*}{$\begin{array}{l}\text { Vérifier l'application } \\
\text { des normes, } \\
\text { améliorer les } \\
\text { conditions d'accès } \\
\text { aux marchés de } \\
\text { qualité }\end{array}$} & \multirow{4}{*}{$\begin{array}{l}\text { Information } \\
\text { sur les marchés }\end{array}$} \\
\hline & Financement & & \\
\hline & Innovation & & \\
\hline & $\begin{array}{l}\text { Information } \\
\text { technique }\end{array}$ & & \\
\hline $\begin{array}{l}\text { Lobbying, } \\
\text { négociation } \\
\text { auprès des } \\
\text { politiques }\end{array}$ & $\begin{array}{l}\text { Négociation pour } \\
\text { améliorer les } \\
\text { infrastructures } \\
\text { (routes, } \\
\text { marchés) }\end{array}$ & $\begin{array}{l}\text { Mobiliser d'autres } \\
\text { acteurs pour des } \\
\text { campagnes de } \\
\text { promotion des } \\
\text { produits agricoles, } \\
\text { négocier des normes } \\
\text { garantissant la } \\
\text { qualité }\end{array}$ & $\begin{array}{l}\text { Règles de } \\
\text { fixation des } \\
\text { prix : par } \\
\text { exemple, } \\
\text { interprofessions, } \\
\text { négociation } \\
\text { sur accords } \\
\text { douaniers }\end{array}$ \\
\hline
\end{tabular}

acteurs de la filière. Il peut néanmoins permettre aux OP d'entrer dans des processus d'apprentissages, en augmentant notamment leurs connaissances des marchés.

Les partenariats sont ainsi utiles et parfois nécessaires, voire indispensables dans un contexte de moyens humains et financiers limités. Cependant ils peuvent se révéler difficiles à construire et à pérenniser pour plusieurs raisons :

- le manque d'information sur les partenaires et donc la difficulté à bien les choisir;

- la difficulté à construire et à maintenir de la confiance, ce qui suppose d'accepter de gagner moins notamment au début de la relation, ce qui n'est pas toujours bien compris par les membres des OP; 


\section{Encadré 20. Étude de cas : les organisations de producteurs de café et les certifications vertes au Costa Rica}

Au Costa Rica, face aux crises récurrentes du marché mondial du café, les coopératives ont mis en œuvre diverses actions stratégiques pour améliorer l'accès de leurs membres aux marchés et rechercher une meilleure valorisation de leur production.

En particulier, les OP costariciennes ont investi dans les certifications telles que commerce équitable FLO Certified, Rainforest, Alliance, UTZ Certified, CAFE Practices. Aujourd'hui, toutes les coopératives costariciennes travaillent avec au moins une certification. Pour ce faire, certaines d'entre elles se sont regroupées dans des consortiums afin de faciliter la gestion et réduire les coûts de la certification, ainsi que pour mutualiser la gestion de la commercialisation des produits. Tel est le cas par exemple du consortium Coocafe, créé en 1989 et comptant aujourd'hui 10 coopératives produisant du café équitable, ou le consortium Suscof, regroupant 6 coopératives et certifié UTZ Certified. Par ailleurs, afin de réduire les coûts de gestion de différentes certifications, certains consortiums ont mis en place des systèmes communs de suivi entre leurs coopératives membres, ce qui leur permet de mutualiser les processus de gestion de l'information nécessaire aux différentes audits de certification.

(d'après Faure G., Le Coq J.F., 2009. Estrategias de las cooperativas cafetaleras frente a los sellos ambientales en Costa Rica)

- le problème de la formalisation. Le passage au contrat écrit, puis le respect de celui-ci peut se révéler difficile dans certains contextes, notamment lorsqu'un document écrit a moins de valeur que la parole donnée.

Ces différents points font ressortir l'importance de l'information et de la communication au sein de l'OP mais également entre celle-ci et l'extérieur, avant la mise en place d'un partenariat et tout au long de la construction de celui-ci (encadré 21).

\section{Les OP et le financement de l'agriculture}

Un meilleur accès aux services financiers est une demande forte et récurrente des producteurs. C'est pourquoi certaines OP se sont spécialisées dans ce service, ou l'ont créé en complément à d'autres services (approvisionnement, commercialisation). 


\section{Encadré 21. Filière et chaîne de valeur}

Les deux notions de filière et de chaîne de valeurs se recoupent mais ne sont pas complètement interchangeables. Elles correspondent à une lecture différente du contexte dans lequel interviennent les OP, qui a évolué avec le temps.

Pendant longtemps, on a privilégié une "analyse éclatée» des différentes étapes entre production et consommation : calculs de marges, ratios coûts/ bénéfices, prix de revient, etc. Généralement, on ne prenait pas ou peu en compte les interactions, on considérait que les producteurs n'étaient pas ou peu concernés par la transformation, la commercialisation.

On est ensuite passé à l'approche «filière classique»: on intégrait les différentes étapes dans une analyse linéaire, reposant sur l'idée que la production répond plus ou moins passivement aux signaux du marché. Ces approches ont été conçues généralement pour piloter l'ensemble d'une filière avec une vision initiale planificatrice.

L'approche «chaîne de valeur» est celle qui est maintenant privilégiée (certains continuent à parler d'approche filière, même si le contenu a évolué). Elle prend en compte le produit à tous ses stades (ainsi que l'amont et l'aval) et s'intéresse à l'ensemble des acteurs et aux relations entre eux. C'est une approche plus dynamique, qui accorde une large place à l'analyse des acteurs et est particulièrement adaptée pour comprendre ce qui se passe localement autour d'un produit, notamment comment se construit sa valeur finale. Ces approches sont davantage orientées pour éclairer des choix stratégiques des organisations économiques (entreprises) au sein de la filière.

Les problèmes en matière de financement sont multiples; selon les difficultés rencontrées par les producteurs, les OP peuvent mener différentes catégories d'actions (tableau 6).

Face à ces multiples possibilités les OP doivent définir leur stratégie en fonction du contexte et des besoins spécifiques de leurs membres. Cette dernière réflexion peut par exemple se fonder sur une série de questions (voir encadré 22).

Quand les OP optent pour l'option de devenir un opérateur de crédit, les OP peuvent rencontrer des problèmes dont certains sont d'ailleurs communs à tous les organismes de crédit.

Le premier écueil est le risque de non-remboursement qui peut être lié :

- à des garanties insuffisantes; une OP peut par ailleurs être plus sensible qu'un organisme purement financier aux problèmes subis par 


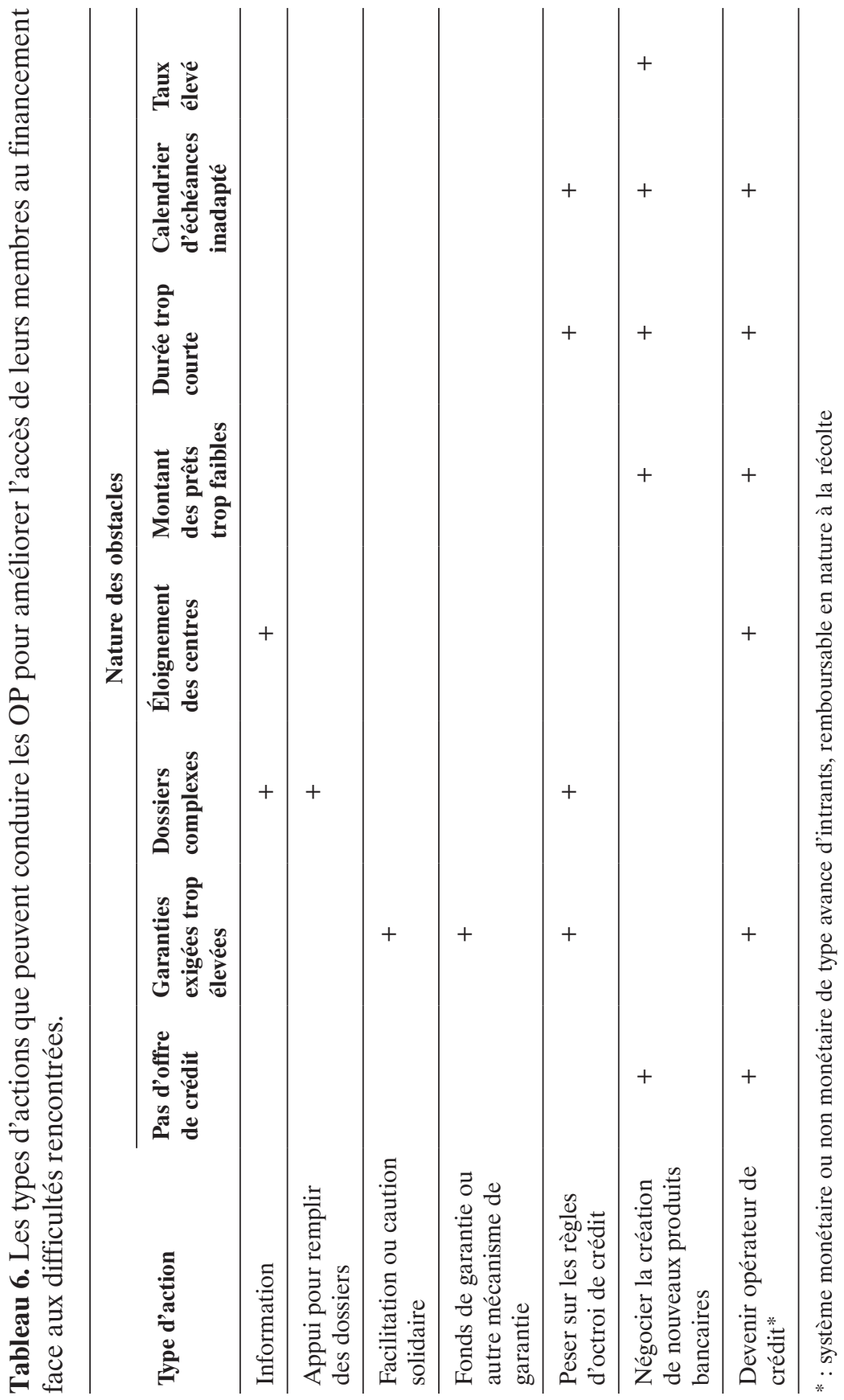


ses membres (accident climatique, sinistre). Si aucun mécanisme d'assurance ou de garantie de type caution solidaire n'a été mis en place, l'ensemble du dispositif peut être mis à mal sans que la bonne foi des bénéficiaires puisse être mise en cause;

- au fait que dans certains cas, les producteurs peuvent se sentir plus libres vis-à-vis de leur organisation et être moins rigoureux dans le respect des échéances; cela peut être aussi l'inverse. La stratégie d'information et de communication de l'OP se révèle essentielle pour peser dans un sens ou l'autre.

Le deuxième écueil est le manque d'un volume de clients suffisant, du fait de la compétition avec d'autres acteurs fournissant des crédits.

\section{Les OP et l'innovation technique}

Linnovation technique est une variable clé de la compétitivité. Les systèmes de recherche, de vulgarisation et de conseil aux producteurs ont connu d'importantes évolutions aux cours des dernières années; avec le retrait des États, des formes de partenariat public-privé se sont mises en place.

\section{Encadré 22. Questions à poser pour choisir les modalités de crédit}

Quel est l'objectif du crédit?

- productif, social.

Quels sont les besoins?

- montant, dates et délais de mise à disposition, durée, date des échéances.

Qui sont les bénéficiaires?

- producteurs (individuels, groupes de caution solidaires), organisations de producteurs.

Quelles sont les ressources disponibles?

- épargne des membres (mutualisme) et des non-membres (activité d'épargne);

- fonds des organismes d'appui (ONG, bailleurs) à travers des subventions, des fonds revolving («ou fonds rotatifs»), des lignes de crédits à taux bonifiés; - ressources venant des marchés financiers (système bancaire à travers des relations commerciales) avec ou non une exigence de garanties (caution de bailleurs, capitaux propres de l'OP, warrantage ou mécanisme de garantie d'un prêt par un stock de produit dont on attend l'augmentation du prix). 
Ce type de service en lien avec la recherche et le conseil relève des biens publics, il est par conséquent assez difficilement gérable par des relations de marché. En outre, le paiement du coût réel de la recherche et de la vulgarisation est impossible pour les producteurs les plus démunis; enfin, après des années de fourniture gratuite de ce service par des agents de l'État, il est difficile de changer les habitudes.

Les OP peuvent contribuer à la production d'innovations et proposer du conseil par le biais de différents types d'actions qui peuvent être combinées, par exemple : actions de formation ciblées, discussions de groupe (groupes se réunissant régulièrement ou occasionnellement), visites de fermes, champs-école (ou Farmer Field School). Ces dispositifs d'apprentissage sont fondés sur le suivi de parcelles paysannes de démonstration, sur des visites d'expérimentations et de démonstrations conduites par les organismes de recherche ou de vulgarisation, ou sur des expérimentations conduites par l'organisation elle-même.

Pour mettre en œuvre cette fonction d'appui-conseil, les OP ont diverses options qui correspondent à différents degrés d'internalisation ou d'externalisation des services :

- elles peuvent s'appuyer sur leurs ressources humaines internes (membres ou salariés) ou mettre en place une équipe spécialisée de techniciens salariés;

- elles peuvent nouer des partenariats spécifiques, par exemple définir des programmes de recherche-développement ou de recherche-action en partenariat avec d'autres acteurs publics ou privés, passer des contrats avec des prestataires publics ou privés;

- elles peuvent mettre en place ou favoriser la mise en place d'un centre de service plus ou moins spécialisé dans la fonction de conseil (voir encadré 23);

- elles peuvent également travailler à canaliser les appuis techniques vers les groupes qu'elles représentent. L'existence d'OP - et leur dynamisme - est également un critère pour l'installation de projets d'appui dans une zone particulière.

Dans tous les cas, la fonction de conseil demande un investissement qui suppose de trouver des sources pérennes de financement alors que les producteurs sont en général peu enclins à payer ces services de manière directe, même quand cette fonction est assurée, totalement ou en partie, par des producteurs membres.

La contribution des OP à la création et à la diffusion des innovations techniques devrait continuer à gagner en importance du fait de la prise 


\section{Encadré 23. Étude de cas : les centres de services}

Une expérience de centres de services aux exploitations a été initiée au début des années 2000 dans la zone de l'Office du Niger au Mali. Il existait déjà dans la zone des centres de prestations de services (CPS) qui proposaient aux OP adhérentes un ensemble de services de conseil en gestion, financiers et juridiques. Ces centres ont étendu leurs prestations au conseil de gestion aux exploitations pour répondre aux besoins exprimés par les membres des OP. Le service est assuré par des conseillers organisés en association sous contrat avec les centres de prestations de services. Les cotisations des adhérents ont couvert une part croissante des frais réels de fonctionnement de ces centres, mais l'autonomie complète n'a pas été atteinte.

Les centres de services agricoles lancés à Madagascar en 2010, sous l'égide du ministère de l'Agriculture de l'Élevage et de la Pêche et avec l'appui de plusieurs bailleurs de fonds, répondent à une logique différente : ils n'ont pas pour fonction de rendre directement des services mais celle de faciliter l'accès des producteurs aux services. Leur rôle est plutôt celui d'intermédiaires entre utilisateurs et prestataires : identification des besoins; appui à la contractualisation; aide à la recherche de financements complémentaires, une partie au moins des frais étant toujours à la charge du producteur.

de conscience générale de la non-efficacité et de la non-efficience globale des systèmes nationaux de recherche et de vulgarisation. La mise en place dans de nombreux pays de dispositifs consultatifs d'orientation de la recherche répond à ce constat : les OP y sont généralement associées soit en tant que membres des instances d'orientation, de programmation ou d'affectation des moyens, soit associées à l'établissement d'agendas de recherche financés par des fonds compétitifs.

Le rôle des OP ne se limite pas à la diffusion de l'innovation à travers la fonction de conseil et à l'orientation du travail des chercheurs. Elles peuvent contribuer également au développement même de l'innovation par leur participation à un travail conduit par des organismes de recherche, ou dans le cadre de volets d'activité internes; ces divers dispositifs relèvent de la recherche-action, ou de la recherche-action participative.

\section{Les OP et la gestion de biens partagés}

La gestion de biens partagés, qu'il s'agisse de ressources naturelles (eau, forêts) ou plus généralement des biens publics locaux dans une démarche de développement territorial ou de développement local, 
met en jeu des activités particulières, qui sont à la limite du champ spécifique d'intervention des OP car la population concernée dépasse en général celle de leurs membres.

Elles peuvent cependant être amenées à s'impliquer dans le développement local ou dans la gestion des ressources naturelles pour plusieurs raisons :

- acquérir de la légitimité auprès de la population locale;

- sécuriser leurs activités principales quand celles-ci nécessitent des infrastructures nouvelles, ou quand le manque de coordination compromet les activités de toute une communauté;

- répondre à la demande de l'État ou d'autres acteurs, dans le cadre de démarche de transfert de compétences à la société civile.

Il faut souligner que les ressources naturelles étant des biens publics (locaux ou globaux), leur gestion - et plus généralement la gestion d'un territoire - est porteuse d'enjeux particuliers qui ne peuvent être résolus par la coordination marchande. Les problèmes spécifiques rencontrés par les OP impliquées dans ce type d'activités sont d'établir et de faire respecter des règles collectives par les populations locales et par les populations extérieures qui ne font pas nécessairement partie de leurs membres.

La nature des activités et le degré d'implication des OP dans la gestion de biens partagés peuvent prendre des formes diverses allant de la simple participation à la conception du projet de territoire, voire à la définition et à la mise en œuvre d'un projet communautaire de développement local. Des compétences particulières sont alors nécessaires : capacité à mobiliser la population locale, bonne compréhension du cadre institutionnel et juridique de la gestion publique locale (rédaction et gestion de contrats publics-privés).

Pour des OP dont le premier objectif est de fournir des services à leurs membres pour le développement de la production, il peut être risqué de s'investir dans des processus de développement local; le risque est en effet de se disperser dans des activités et de perdre sa spécificité. C'est pourquoi, avant de s'engager sur ce terrain, les organisations doivent s'interroger et développer une vision claire des opportunités et des risques représentés par cette évolution.

\section{Pourquoi des partenariats? Quels partenariats?}

Le partenariat est une des formes de relations entre les OP et d'autres acteurs. C'est une forme d'accord volontaire entre deux 
parties, qui peut être plus ou moins formalisé. Cela peut aller du contrat écrit précisant un ensemble de droits et devoirs réciproques et prévoyant les modalités d'arbitrage et de règlement des conflits éventuels, à un accord informel dont les clauses sont vagues. Entre ces deux extrêmes, il existe toute une gamme de possibilités qui se caractérisent par leur degré de formalisation et par la précision des clauses. De manière générale, les partenariats sont un moyen pour une organisation d'accéder à des ressources (humaines, financières, information) qui lui sont nécessaires pour rendre un service de qualité à ses membres.

Les partenariats se construisent de façon diverse et répondent à des questions différentes selon les domaines d'action concernés, comme nous l'avons vu dans les domaines de la mise en marché des produits agricoles, du financement de l'agriculture, de la recherche et la conception d'innovations techniques, du conseil et de la gestion des ressources naturelles.

Ils se construisent dans la durée, et, pour être viables, supposent souvent le partage de mêmes valeurs ou au moins des affinités, mais ils peuvent aussi être plus ou moins opportunistes. Le partenariat repose sur la confiance entre les partenaires. Cependant, même quand la confiance existe, il est souvent utile de garantir l'accord par un processus de validation auprès d'une tierce partie.

Les OP ne peuvent pas ou ne doivent pas tout faire : elles ont à s'interroger sur leurs avantages comparatifs par rapport à d'autres acteurs, avant de décider de mettre en œuvre ou non une activité ou un service à leurs membres ou à la population locale. Quel que soit leur choix, elles ont un rôle important à jouer dans la coordination de l'offre de service locale soit en orientant l'offre (fonction de représentation), soit en fournissant directement le service (fonction de prestation) ou en agissant comme médiateur entre les prestataires publics ou privés et les producteurs.

\section{Pour aller plus loin}

Faure G., Gasselin P., Triomphe B., Temple L., Hocdé H., 2009. Innover avec les acteurs $d u$ monde rural. La recherche-action en partenariat. Agricultures tropicales en Poche, Quæ, CTA, Presses agronomiques de Gembloux, 224 p. 
Lothoré A., Delmas P., 2009. Accès au marché et commercialisation des produits agricoles. Valorisation d'initiatives de producteurs. Inter-réseaux Développement rural, CTA, AFD. http:// www.inter-reseaux.org/ressources-thematiques/ressources-sur-la/ capitalisation-d-experiences-de/article/acces-au-marche-et

Dugué M.J., Le Coq J.F., 2006. Pedagogical materials on farmers organisations and farmers organisations support. CIRAD, Montpellier, 84 p.

Dugué M.J., Le Coq J.F., Berthomé J., Mercoiret M.R., Pesche D., 2006. Farmers' organization tool kit: Review of worldwide case studies. CIRAD, Montpellier, 108 p.

Dugué M.J., Liagre L., David-Benz H., Minla J., 2006. Kit de formation. Analyse des filières riz par les organisations professionnelles d'Afrique de l'Ouest. IRAM, CIRAD, CIEPAC, APM, ministère français des Affaires étrangères, DGCID-DCTEPS, téléchargeable sur www.iramfr.org (filières, organisation des acteurs). http://www.ruralfinance.org/

Cerise - Comité d'échange, de réflexion et d'information sur les systèmes d'épargne-crédit, IRAM, 2005. Guide opérationnel d'analyse de la gouvernance d'une institution de microfinance. FIDA, GTZ, IRAM, Paris, téléchargeable sur www.cerise-microfinance.org/IMG/pdf/ guide_ope_analyse_gouvernance-2 


\section{$\sqrt{\text { 8. La participation des }}$ organisations de producteurs à la définition des politiques publiques}

Les OP doivent-elles, et peuvent-elles influer sur les politiques publiques et comment? La plupart des décideurs politiques (soutenus dans ce sens par les bailleurs de fonds institutionnels) admettent désormais qu'il est utile, voire nécessaire, d'associer les acteurs à la définition des politiques. C'est pourquoi les OP sont de plus en plus sollicitées pour contribuer à la définition des politiques agricoles ou de développement rural; même si ce n'est pas leur objectif premier elles savent que, si elles ne sont pas capables de répondre à cette invitation, d'autres le feront à leur place. Dans certains cas, ce sont les OP elles-mêmes qui ont pris l'initiative de porter la voix des ruraux auprès des politiques. Or, dans ce champ d'action particulier, les OP sont confrontées à plusieurs enjeux que nous analyserons successivement dans ce chapitre pour en tirer des enseignements en termes d'action.

\section{De nombreuses OP sont concernées par la définition des politiques}

La multiplication des groupements à la base et leur progressive articulation dans des ensembles plus larges (unions, fédérations) constituent les deux caractéristiques principales des dynamiques récentes d'organisation en milieu rural (voir chapitre 1).

La plupart du temps, les regroupements d'organisations à l'échelle nationale ou supranationale se font autour de revendications généralistes. Ils représentent une diversité d'intérêts agricoles et ruraux, et agissent pour influencer les politiques agricoles et rurales en faveur des intérêts de leurs membres.

Il arrive que ces organisations soient spécialisées, autour d'un produit par exemple (organisation nationale des producteurs de riz, de coton). 
Leur préoccupation est alors principalement économique mais elles sont également soucieuses de la défense des intérêts des producteurs autour de ce produit. Elles cherchent à s'assurer d'un environnement favorable à leur activité et remplissent dans cet objectif des fonctions de représentation des intérêts de leurs membres.

Il n'est donc pas pertinent de maintenir une distinction trop rigide entre, d'une part, des organisations qui rendent des services à leurs membres (économiques, techniques) et, d'autre part des organisations qui assurent la défense des intérêts et auraient davantage un rôle politique. D'abord parce que les premières sont souvent amenées à intervenir sur les politiques sectorielles. Ensuite, dans de nombreuses situations où les OP sont moins spécialisées et assurent plusieurs fonctions, les regroupements engagés dans l'élaboration des politiques publiques combinent, souvent au sein des mêmes mouvements, une logique de fourniture de services aux agriculteurs et aux ruraux avec une logique de représentation et d'influence politique.

Pour une organisation ou un mouvement d'organisations, s'engager dans l'influence des politiques suppose la construction d'une stratégie de défense de causes qui dépasse souvent la somme stricte des intérêts des membres. Cela nécessite souvent que l'organisation et ses responsables produisent une analyse de la place de l'agriculture dans l'économie et dans la société, pour situer leurs revendications et leurs propositions dans un contexte plus global face à leurs interlocuteurs.

En effet, l'articulation entre logiques économiques et politiques et la prise en compte des dimensions sociales, territoriales, voire culturelles, sont souvent à l'origine des coalitions d'acteurs qui parviennent à influencer les politiques. Les OP sont rarement seules dans ces processus. Elles ne sont pas des acteurs isolés dont la réussite dépendrait uniquement de leurs capacités internes. Le plus souvent, elles ont des relations avec d'autres acteurs (ONG, universités, services techniques) avec qui elles peuvent s'allier pour soutenir leurs stratégies d'influence.

\section{Les OP cherchent à influencer les politiques dans un contexte qui évolue}

Comprendre le rôle que jouent les OP dans l'influence des politiques suppose aussi de comprendre les évolutions des espaces politiques nationaux, leurs principales caractéristiques mais aussi les transformations institutionnelles plus générales liées à la globalisation. 
Depuis les années 1980, les États se sont transformés sous l'influence combinée des politiques économiques et institutionnelles qui leur ont été largement imposées par la communauté internationale (bailleurs de fonds, agences de coopération). Les politiques d'ajustement structurel et, plus largement, les politiques d'inspiration néolibérale ont contribué à affaiblir les États et ont encouragé l'implication des acteurs non étatiques dans les décisions publiques, tels que le secteur privé marchand et la société civile.

Beaucoup de pays ont amorcé un processus de décentralisation mais rares sont aujourd'hui les collectivités locales qui jouissent d'une autonomie politique et financière suffisante pour pouvoir influencer des choix de politique pour l'agriculture et le monde rural, ces orientations restent encore largement déterminées par les décisions nationales.

On observe aussi la construction d'ensembles régionaux regroupant plusieurs pays voisins (UEMOA, Cedeao, Sadec, par exemple en Afrique; Mercosur en Amérique latine) et constituant progressivement des espaces économiques importants pour les échanges agricoles. La prise en compte de nombreux enjeux suppose aussi des actions et des engagements sur le plan international : commerce, environnement, conflits.

La multiplication des échelons de gouvernance tend à complexifier les processus de décision politique et à multiplier les acteurs qui $\mathrm{y}$ sont impliqués. Malgré cette complexification des modes de décision publique, l'échelon national reste encore déterminant dans la construction des intérêts des ruraux et dans le rôle qui leur est accordé pour peser sur les décisions (de Janvry et Sadoulet, 2003).

On peut également souligner la multiplication des instances et des agendas influençant l'avenir des producteurs et des ruraux. Les décisions concernant le développement agricole et rural étaient, historiquement dans de nombreux pays, largement incluses dans un seul agenda agricole, souvent maîtrisé par un ministère et quelques acteurs. Depuis les années 2000, les politiques publiques influant le monde rural sont le fruit de plusieurs agendas politiques (commercial, environnemental, social) sous la responsabilité de différentes administrations telles que le ministère du commerce (qui régule les échanges) mais également des plateformes de définition des normes privées ou publiques, le ministère de l'environnement, le ministère des infrastructures. Sur le plan international, cette tendance se confirme également avec la multiplication des instances influençant potentiellement l'avenir des agriculteurs (agenda sur le changement climatique, le 
commerce, la sécurité alimentaire, les ressources en eau, la biodiversité). Cette multiplication des cadres de discussion et des agendas rend difficile pour les OP, dont le nombre de leaders est par essence limité, la mise en place d'un suivi efficace. La profusion de négociations peut conduire en outre à une dispersion. Des choix stratégiques sont alors nécessaires pour privilégier le suivi d'un agenda jugé prioritaire (au risque de passer à côté de négociations importantes pour le moyen terme), ou pour construire des alliances permettant de suivre la diversité des négociations en cours.

Lexemple du CNCR (encadré 24) montre bien que le jeu démocratique à l'échelle du pays peut avoir des conséquences sur les OP.

\section{Encadré 24. Les relations du Conseil national de concertation et de coopération des ruraux (CNCR) avec le pouvoir politique sénégalais}

Le CNCR a été créé en 1993 pour représenter la diversité des organisations rurales du Sénégal. Il constitue depuis cette époque un interlocuteur des pouvoirs publics pour la négociation des politiques agricoles. Cette fonction de représentation ne va pas forcément de soi et est remise en cause régulièrement par le pouvoir politique. C'est le cas en particulier depuis 2000 où l'alternance politique a distendu les relations qui s'étaient construites progressivement. Malgré un contexte plutôt hostile, le CNCR a su influencer le processus d'élaboration de la loi d'orientation agro-sylvo-pastorale en 2004. Il a continué à jouer son rôle de représentation des intérêts des ruraux, dans un contexte politique de plus en plus difficile. En 2010, le CNCR a organisé un forum sur la capacité des exploitations familiales à nourrir le Sénégal. Préparée avec la Fédération des ONG du Sénégal (FONGS), qui est un de ses membres fondateurs et un de ses plus actifs soutiens à l'échelon national, cette manifestation a permis au CNCR de trouver un écho dans la société sénégalaise et a renforcé les appuis dont il bénéficie sur le plan international.

\section{L'influence politique se joue d'abord au niveau national}

Après la période des ajustements structurels, le rôle de l'État est de nouveau reconnu comme important pour initier et accompagner les réformes dans le secteur agricole et le monde rural. Limportance croissante des échelons supranationaux ne doit pas faire oublier que 
ce sont les interactions et les négociations entre les États qui régissent la grande majorité des institutions et des accords internationaux.

Linfluence des OP se fait sentir sur trois grands types de politiques nationales :

- les politiques sous-sectorielles, autour de filières de produits agricoles ou d'élevage. Les exemples sont nombreux d'organisations qui ont pu faire entendre avec un relatif succès leurs points de vue (coton au Mali et au Bénin, pomme de terre en Guinée, café en Colombie); - les politiques transversales concernant un aspect particulier du monde rural (foncier, services agricoles, financement rural). Là aussi, des OP fortes peuvent jouer un rôle dans la définition des choix et leur mise en œuvre, par exemple lors des réformes des services agricoles (Diaz et al., 2004) ou lors des débats sur le foncier (Benkahla et al., 2011);

- les politiques agricoles et rurales plus globales qui tracent les orientations générales et les grands choix pour l'avenir. C'est le cas par exemple des lois d'orientation agricoles élaborées au milieu des années 2000 au Sénégal et au Mali, les OP ont joué alors un rôle déterminant grâce à un processus de concertation approfondi.

Plusieurs facteurs sont importants à considérer pour comprendre le rôle des OP dans l'influence des décisions publiques et être en mesure d'agir pour le renforcement de leur capacité dans ce domaine.

Les formes institutionnelles de représentations des intérêts des ruraux à l'échelle nationale peuvent être différentes selon le niveau d'articulation des institutions avec les pouvoirs publics : plus ou moins d'autonomie vis-à-vis de l'État, plus ou moins grande décentralisation dans le fonctionnement.

Les mécanismes de représentation et de défense des intérêts agricoles sont en général dépendants du type de régime politique. En Afrique, les pays de tradition francophone privilégient une forme de représentation et de défense des intérêts s'exerçant dans un dialogue et une confrontation avec l'administration et les autorités politiques (voir encadré 25). Les pays de tradition anglo-saxonne semblent privilégier des modalités et des canaux de défense des intérêts moins exclusifs et plus ouverts avec un rôle des parlementaires et des ONG (voir encadré 26). La représentation des intérêts des ruraux au Sénégal et au Mali par exemple est rendue plus aisée qu'au Tchad ou au Togo où les régimes politiques sont moins ouverts au dialogue avec la société civile. 


\section{Encadré 25. Étude de cas : un exemple de la stratégie d'influence des organisations de producteurs sur la filière coton au Burkina Faso}

L'Union nationale des producteurs de coton du Burkina Faso (UNPCB) est une organisation paysanne cotonnière nationale très insérée dans les mécanismes de gestion de la filière. Elle possède $10 \%$ du capital de la société cotonnière nationale, la Sofitex et participe activement à l'interprofession qui gère l'ensemble de la filière. C'est une forme de représentation des intérêts où l'organisation des producteurs est en partie liée à ses partenaires. Lavantage est qu'elle est au cœur des mécanismes de décision pour la filière, qu'elle peut ainsi mieux influencer les règles de fonctionnement de la filière; l'inconvénient est que son indépendance peut être menacée en particulier si son financement dépend d'accords entre les partenaires de la filière. L'État pèse aussi beaucoup dans les décisions concernant la filière et il a une influence certaine sur les choix de l'organisation.

\section{Encadré 26. Étude de cas : au Kenya, les rôles des parlementaires et du secteur privé}

Au Kenya, on a pu constater, après le changement de présidence (en 2002), l'importance accrue des parlementaires dans le processus de définition des politiques. Plusieurs commissions ou groupes parlementaires ont défendu explicitement certaines options, comme la privatisation de la filière thé par exemple. Le parlement s'est doté d'une expertise spécifique lui permettant de mieux dialoguer avec les autres acteurs (en particulier l'Administration) dans le processus d'élaboration des politiques, avec un soutien visiblement important de certains bailleurs de fonds tels que l'USAID, le DFID. De la même façon, les ONG sont reconnues comme mieux habilitées à représenter les intérêts des ruraux pauvres, par exemple, les organisations paysannes. Lorganisation faîtière des producteurs et des ruraux, la KENFAP, est membre du KEPSA, l'Alliance kenyane pour le secteur privé, ce qui lui donne une légitimité plus grande au moment de négocier avec d'autres ministères que celui de l'agriculture. Le système institutionnel kenyan semble ainsi plus ouvert et multi-institutionnel que dans certains pays francophones où l'on a le sentiment d'assister à un face-à-face, plus ou moins fonctionnel, entre les OP et les pouvoirs publics.

Lautonomie politique et financière des mouvements d'OP est importante. La première est plus souvent atteinte que la seconde et la diversification des partenariats est un gage d'autonomie pour les OP engagées dans le champ politique (voir chapitre 7). 
Dans tous les cas, la capacité d'influence des organisations de producteurs sur les politiques est souvent liée à leur capacité à forger des alliances à la fois au sein du monde rural (exemple du CNCR au Sénégal, encadré 24), ou à s'insérer dans les mécanismes de décision formelle (exemple de la UNPCB au Burkina Faso, encadré 25), ou encore à s'allier à d'autres acteurs de la société civile ou du secteur privé marchand (exemple du Kenya, encadré 26). Pour cela, la capacité des OP à utiliser les ressorts des processus de démocratisation est un atout souvent déterminant (rôle des médias pour soutenir la cause des ruraux, des parlementaires).

Peser sur les politiques suppose pour les OP une certaine maîtrise des contraintes logistiques et de communication qui sont encore très fortes dans beaucoup de situations : une coalition d'OP engagée dans un travail d'influence sur la décision publique doit pouvoir communiquer rapidement avec ses membres. Dans ce domaine, le rapide essor des nouvelles technologies de l'information et de la communication (Internet, téléphone cellulaire) est un atout important même si ces outils ne peuvent pas remplacer les concertations de face-à-face nécessaires à la vie d'un mouvement. C'est un domaine où des appuis extérieurs sont souvent utiles.

Tous les éléments évoqués ci-dessus ont leur importance pour assurer aux OP un rôle effectif dans l'influence sur les politiques. Mais l'élément clé est sans doute le rôle déterminant que sont appelés à jouer les leaders de ces organisations. Les responsables des OP ont un rôle central dans la construction d'un projet stratégique pour leurs organisations (voir chapitre 4). Ils jouent notamment un rôle clé pour s'assurer de la construction et de la solidité des alliances, veiller à la prise en compte des intérêts souvent diversifiés des ruraux qu'ils représentent et enfin améliorer les conditions du dialogue politique (cadres de concertation, agendas).

\section{L'intervention des OP est nécessaire à l'échelle supranationale}

Si les principaux efforts des OP consistent à influencer les politiques agricoles et rurales dans leurs pays, on voit aussi se créer des réseaux d'OP issues de plusieurs pays et cherchant à influencer des décisions prises au-delà de l'espace national. On distingue deux échelles :

- l'échelle sous-régionale où sont élaborées des politiques agricoles qui ont une importance croissante sur les transformations de 
l'agriculture et du monde rural dans les pays du Sud (UEMOA, Cedeao, Mercosur);

- l'échelle internationale qui est le lieu des négociations commerciales, des accords sur les aspects environnementaux, etc. qui ont des répercussions non seulement sur le commerce international mais aussi sur les agricultures locales.

Dans tous les cas, l'implication récente de réseaux d'OP à ces échelles a connu des succès indéniables. Ils ont été permis par la capacité de ces réseaux à relier et à mobiliser des OP de différents pays autour de thèmes fédérateurs, et à construire des coalitions larges incluant d'autres acteurs de la société civile.

Comme cela s'est fait en Europe avec la mise en place du marché commun agricole, il faut souligner l'importance de nouvelles alliances entre les plateformes nationales d'organisations de producteurs ruraux

et leurs États. Les OP d'un pays se retrouvent confrontées à leur État dans la négociation des politiques nationales mais doivent établir avec celui-ci des alliances pour défendre des causes sur le plan international. Pour les OP, la multiplication des échelles de gouvernance (locale, nationale, internationale) se traduit concrètement par la multiplication des espaces de décision et de pression : la fragmentation des espaces de décision publique rend complexe le travail d'influence et nécessite de nouvelles alliances. L'implication des OP dans des négociations internationales peut renforcer leur poids sur le plan national, ce que certains appellent l'effet boomerang (Newell et Tussie, 2006).

En matière d'influence sur les politiques, les OP doivent adapter leur structuration à l'échelle de leurs interlocuteurs, ce à quoi s'emploient les OP africaines (encadré 27).

\section{Constats, interrogations et pistes d'avenir}

En matière de participation des OP à l'élaboration des politiques, on rencontre encore deux écueils : la difficulté de dialoguer et de négocier, et la gestion des flux d'information.

\section{ID Les conditions d'un dialogue et de négociations équilibrés sont rarement réunies}

Ce premier constat conduit aux interrogations suivantes : comment améliorer les conditions de ce dialogue pour éviter des situations de 


\section{Encadré 27. Mise en place progressive de réseaux supranationaux d'organisations de producteurs en Afrique}

Dans les années 1990, plusieurs rencontres régionales avaient permis à des OP de différents pays d'Afrique de l'Ouest de se rencontrer. En 2000, la perspective de mise en place d'une politique agricole à l'échelle de l'UEMOA précipite la création d'un réseau des organisations paysannes et de producteurs d'Afrique de l'Ouest, le ROPPA. Depuis, ce réseau s'est investi dans les négociations pour influer sur les politiques agricoles à différents niveaux : UEMOA en 2001-2002, puis CEDEAO en 2005 et, en 2007, accords de partenariat économique (APE) entre l'Union européenne et les pays d'Afrique de l'Ouest.

Pendant cette même période, plusieurs autres réseaux régionaux d'OP se sont créés, souvent dans le même but de représenter les producteurs dans des processus de définition de politiques agricoles à l'échelle régionale. Il s'agit en particulier de l'Eastern Africa Farmers Federation (EAFF en 2001) et de la de Plateforme régionale des organisations paysannes d'Afrique centrale (PROPAC en 2004).

Par ailleurs, sur la lancée du succès remporté à Cancún, des producteurs spécialisés se sont aussi constitués en organisation au niveau continental avec la création en 2005 de l'Association des producteurs de coton africains (APROCA).

Enfin, depuis 2008, une plateforme panafricaine des organisations paysannes et de producteurs agricoles s'est mise en place, avec l'objectif de parler d'une seule voix dans les instances continentales, et notamment au NEPAD.

désengagement ou de conflits? Comment réduire les asymétries entre acteurs appelés à participer à la décision publique?

Le discours communément tenu par les acteurs du développement tend à accorder de l'importance aux processus de dialogue et à la participation. Force est pourtant de reconnaître que les OP s'expriment de plus en plus mais ne sont pas forcément entendues pour autant.

Lévolution des relations internationales modifie les relations entre les acteurs d'un même pays. Ainsi, en Amérique latine, la mise en place des accords de libre-échange avec les États-Unis (ALCA) conduit à un rapprochement entre certains gouvernements et leur société civile, dont les mouvements sociaux ruraux (Newell et Tussie, 2006).

Les débats de politiques, en particulier sur le plan international, sont très techniques, ce qui tend à accorder une place prépondérante aux 
spécialistes (par exemple aux juristes), occultant ainsi les dimensions réellement politiques des questions traitées. Paradoxalement, l'abondance d'information s'accompagne d'une opacité croissante des processus de décision.

En dehors des processus formels de débat public, des acteurs puissants agissent de façon discrète pour peser sur les décisions (acteurs du secteur privé, acteurs religieux). Il paraît donc important, pour limiter ces pratiques, que puissent se mettre en place des mécanismes d'institutionnalisation du dialogue selon des modalités négociées avec les principaux acteurs, dont les OP.

Il est aussi important de renforcer les capacités des administrations à organiser la concertation mais aussi à se sentir engagées envers les décisions prises et à assurer le suivi de leur mise en œuvre.

\section{ID De grandes quantités d'informations circulent rapidement grâce aux nouvelles technologies}

De ce second constat résulte l'enjeu suivant : comment faciliter le traitement de cette information abondante, la transformer en connaissances intelligibles et utiles pour les acteurs engagés dans les processus d'élaboration des politiques, et en particulier pour les OP qui souhaitent les influencer?

Face à la profusion d'informations qui ne sont pas toujours adaptées à leur besoin, il est important pour les OP de produire leurs propres informations et connaissances. En ayant recours à une expertise qualifiée et indépendante - choisie par les organisations elles-mêmes les OP se dotent d'un moyen clé de leur travail d'influence sur les politiques.

Des observatoires indépendants ou associant plusieurs acteurs concernés (État, OP, ONG, universités) peuvent aussi jouer un rôle pour améliorer la connaissance des transformations en cours en milieu rural et faciliter ou influencer les décisions. Des recherches sur l'impact des politiques agricoles et rurales peuvent aider les choix de politique en apportant des références indépendantes et argumentées sur les impacts des politiques antérieures.

Pour comprendre leur environnement et s'appuyer sur leur expérience, afin de générer des propositions argumentées en matière de politiques publiques, les organisations de producteurs ruraux doivent 
disposer de sérieuses capacités d'analyse. L'éducation formelle - dont l'impact se mesure à long terme -, et les formations professionnelles et continues - dont les impacts sont de court et de moyen terme - sont pour cela des instruments importants si elles sont adaptées, maîtrisées par les OPR. La participation effective des ruraux dans les processus d'élaboration et de mise en œuvre des politiques qui les concerne entraîne une plus grande pertinence et efficacité de ces politiques, une plus grande appropriation. On peut considérer que la représentation des intérêts des ruraux est donc un bien quasi public (de Janvry et Sadoulet, 2004). On peut aussi considérer que l'existence de cadres de dialogue et un meilleur équilibre des négociations entre acteurs sont des facteurs importants de paix sociale. Ces deux arguments militent en faveur de la mise en place de mécanismes de financement durables des OP qui leur permettraient de bénéficier de soutiens publics dans la durée (nationaux comme internationaux) tout en préservant leur autonomie politique.

Rappelons qu'il y a parfois loin de la définition d'une politique à la mise en œuvre des textes d'application qui la rendent effective. Il ne suffit pas de négocier des orientations, il faut également s'assurer qu'elles se traduisent en mesures concrètes, puis suivre leur application. Cela implique pour les OP de rester mobilisées sur les questions de politique de manière permanente, à travers par exemple des dispositifs de veille à l'échelon national.

\section{Pour aller plus loin}

Benkahla A., Hrabanski M., Pesche D., Ba C.O., 2010. L'implication des organisations de producteurs dans les politiques publiques : enseignements issus de la capitalisation du processus de réforme foncière et de négociation des APE au Sénégal. Dakar, IPAR, CIRAD, 32 p. http://www.ipar. sn/IMG/pdf/Livret_formation_capitalisation_foncier_et_APE.pdf

Chaboussou A., Ruello M., 2007. Processus de concertation pour l'élaboration d'une politique publique. La Loi d'orientation agro-sylvo-pastorale du Sénégal, 2007. Dakar, ISRA, CIRAD, 59 p.

CIRAD, IPAR, 2011. L'implication des OP dans les politiques au Sénégal. Rapports, policy briefs, livret pédagogique. http://www.interreseaux.org/groupes-de-travail/dernieres-capitalisations-menees/ article/l-implication-des-op-dans-les 



\section{$\sqrt{\text { 9. Le diagnostic des }}$ organisations de producteurs}

Dans ce chapitre, nous reviendrons d'abord sur les raisons qui peuvent motiver la réalisation d'un diagnostic, nous énoncerons ensuite quelques principes généraux sur la démarche avant de proposer un petit nombre d'outils adaptés aux différents contextes de diagnostic d'OP.

\section{Les objectifs du diagnostic}

Le diagnostic d'une OP peut être réalisé de l'extérieur ou correspondre à un exercice plus endogène.

Une analyse externe peut, par exemple, être initiée par un partenaire désireux d'appuyer une OP ou de travailler avec elle dans le cadre d'un projet. Le diagnostic, réalisé avant d'engager des actions, vise à en renforcer la pertinence et l'efficacité. Bien entendu, la démarche n'a de sens que si l'OP (élus, membres et salariés) en accepte le principe et s'y implique.

Le diagnostic peut aussi avoir comme objectif principal d'améliorer le fonctionnement et les activités d'une OP. Il peut notamment correspondre à la première étape de l'élaboration d'un plan stratégique (voir chapitre 4). La démarche est alors plutôt conduite en interne, et s'apparente à une auto-évaluation. Même dans ce cas, le diagnostic ne doit pas se limiter à la seule vision des membres, et l'organisation peut avoir recours à une expertise externe, pour peu qu'elle en contrôle les termes de référence et le choix.

Lexercice du diagnostic peut enfin correspondre à un travail de classification ou de typologie. Un service d'appui aux OP ou un projet souhaitant travailler avec un grand nombre d'organisations peut avoir besoin de cibler ou d'organiser ses interventions. Classification et typologie répondent à des besoins différents (voir encadré 28). Pour une classification, il s'agit de disposer de données pour quantifier des besoins, évaluer un impact potentiel. Une typologie répond à une problématique spécifique et est plus orientée vers la compréhension et l'analyse qualitative. 


\section{Encadré 28. Classification ou typologie}

La classification consiste à placer les différentes organisations dans des catégories préétablies bâties sur quelques critères (origine de l'organisation, activité).

Une typologie suppose de préciser une question de départ à laquelle on veut répondre : cela conduit ensuite à construire des types idéaux pour réduire la complexité du processus étudié et chercher à répondre à la question qui a été formulée.

Par exemple, les catégories d'OP généralement distinguées sont : des OP féminines, masculines et mixtes, des OP d'élevage ou agricoles. Construire une typologie est utile pour répondre à des questions diverses. Par exemple, on cherche à comprendre pourquoi certaines remboursent bien leurs crédits et d'autres non. On va alors analyser la diversité des situations rencontrées et bâtir une typologie en combinant des critères qui semblent avoir un sens pour expliquer cette différence dans le rapport au crédit : ancienneté de l'organisation, degré de spécialisation, niveau de formation des responsables.

(d'après Pesche, 2001)

\section{Quelques principes généraux}

Rappelons que la manière de conduire le diagnostic dépend bien évidemment des objectifs et du contexte mais aussi souvent de facteurs très pratiques comme le temps, les moyens et les ressources humaines dont on dispose.

La méthode employée pour élaborer le diagnostic et les conditions dans lesquelles il a été réalisé influent sur le résultat : il n'y a donc pas un diagnostic juste par opposition à tous les autres. Un diagnostic est conduit avec un objectif donné; les résultats obtenus ne peuvent donc pas être utilisés tels quels dans un autre cadre, même s'ils constituent toujours une source d'information utile.

Un diagnostic est réalisé à un moment donné et n'a pas une valeur définitive : il est donc obligatoirement daté. Cela ne veut pas dire qu'il ne s'agit que d'une photo instantanée, un diagnostic prend généralement en compte les aspects dynamiques; on parle d'ailleurs de parcours biographique d'une organisation. L'existence d'un tel diagnostic peut s'avérer très utile par la suite au moment d'évaluer les avancées ou les difficultés d'une organisation : il sert alors de référence précieuse. 
La notion de diagnostic se rapproche de l'idée d'évaluation. Un diagnostic n'est pas seulement descriptif, il inclut aussi une part d'analyse. Le plus souvent, il s'agit de comprendre l'organisation dans son environnement, dans l'optique d'en améliorer le fonctionnement et, par exemple, d'accroître son efficacité à remplir les rôles qu'elle s'est fixée. Parler d'évaluation rajoute une dimension de jugement par rapport à des critères, ce que ne fait pas forcément un diagnostic.

Il faut aussi être attentif au fait que les outils et méthodes ne sont pas neutres, ce sont des instruments qui véhiculent une vision, un parti pris, un angle d'analyse particulier. Il importe de ne pas tomber dans le piège de la fascination des outils et de rester conscient que faire un diagnostic, c'est se donner une grille de lecture de la réalité qui nécessairement va la déformer.

Un diagnostic approfondi ne peut pas se limiter à une analyse interne de l'organisation. Il s'intéresse aussi aux acteurs avec qui l'OP est en relation et à la nature de ces relations et, plus largement, aux enjeux auxquels fait face l'organisation, dans son environnement proche et lointain.

Dans les années 1980-1990, alors que de nombreuses organisations locales voyaient le jour, on pouvait les considérer comme des organisations qui étaient en situation d'interface entre les sociétés locales et la société globale. Leur place apparaissait alors assez clairement. Cette vision est désormais moins évidente, car la limite entre la société globale et les sociétés locales est de plus en plus floue. Cela est dû au développement des moyens de communication et d'information, à une mobilité croissante des personnes et au regroupement des OP locales dans des ensembles fédératifs et des réseaux nationaux, voire internationaux. C'est cependant toujours un des rôles des OP que de contribuer à réguler et à rééquilibrer les relations entre les contextes locaux et plus globaux. La légitimité des organisations fédératives prend sa source aussi bien dans les sociétés locales qu'à d'autres échelles (nationale, internationale) où leurs compétences sont reconnues et appréciées.

Plus généralement, au cours d'un diagnostic, on s'interrogera sur :

- la position de l'OP dans la société locale;

- ses relations avec les acteurs qui constituent son environnement;

- son fonctionnement interne.

De même que le diagnostic dépasse les limites spatiales de l'organisation, il doit également être resitué dans son histoire et notamment 
prendre en compte l'évolution du contexte social, économique et politique, ce qui explique qu'il soit daté.

Le travail de diagnostic peut servir dans de nombreuses circonstances mais il amène toujours une organisation à se poser la question de son identité : qui suis-je ? Quelle est mon image ? C'est pourquoi il peut être utile à la communication interne et externe.

\section{Des outils de diagnostic}

Ces principes généraux une fois posés nous allons voir concrètement comment on peut mettre en œuvre un diagnostic dans différentes situations. Attention cependant : compte tenu de tout ce qui a été dit ci-dessus, on comprend bien que ces exemples ne sont pas proposés comme des modèles à appliquer tels quels, mais plutôt comme des pistes pour élaborer les outils spécifiques adaptés à chaque situation.

En termes de diagnostic, on se trouve dans différents cas de figure selon qu'il s'agit;

- d'analyser une OP de façon globale et de s'intéresser à sa dynamique ;

- d'analyser une OP pour répondre à une question spécifique. Par exemple : quels sont les conséquences du désengagement de l'État? Comment améliorer les services rendus aux membres?

- de faire un diagnostic sur un mouvement d'OP (évaluer une ou des unions);

- de comparer ou classer des OP (typologie).

\section{ID Analyse globale d'une OP : quelles informations recueillir et comment?}

Quels que soient les outils utilisés, certaines variables sont toujours prises en compte lors d'un diagnostic, de manière plus ou moins détaillée :

- l'historique de l'organisation, le contexte de sa création, les principales étapes de son développement;

- ses activités et les résultats obtenus;

- son organisation interne (membres, prise de décision, finances);

- les relations avec l'extérieur et avec la société locale. On cherche ici à identifier au moins les acteurs qui interagissent avec l'organisation de façon régulière.

Dans une démarche de diagnostic, on commence en général par recueillir des éléments descriptifs et, dans certains cas, des éléments 
plus analytiques, nécessaires à la compréhension du fonctionnement de l'organisation. Par exemple, dire que l'organisation a pour activité principale la culture et la commercialisation de café ne suffit pas, il faut connaître les volumes produits, commercialisés, les canaux de commercialisation et éventuellement les marges réalisées sur ces activités.

Cette étape de collecte d'information est centrale et conditionne la pertinence et surtout la qualité du diagnostic; cela justifie d'y consacrer le temps et les moyens nécessaires.

Pour certains aspects qu'on ne peut pas quantifier, il est utile de définir des critères et des modalités de caractérisation de ces critères. Les variables prises en compte peuvent être choisies au départ ou en cours de démarche. Dans le cadre d'une démarche d'accompagnement, la construction des critères avec l'OP fait partie intégrante du travail de diagnostic mais aussi de renforcement des capacités : en effet, le simple fait de s'interroger sur les variables importantes à prendre en compte pour analyser son organisation est un exercice très formateur pour un responsable ou un technicien d'une organisation.

\section{ID Où chercher les informations?}

Dans une démarche de diagnostic, les sources d'information sont diverses et complémentaires : déclarations individuelles recueillies par enquête ou entretien auprès de membres ou de partenaires, expression collective (focus groups ou réunion ou entretien de groupe, la composition du groupe doit alors être précisée et ajustée afin de capter les différences de perception ou d'évaluation, et éviter l'inhibition de certains individus), documents officiels ou non (statuts, comptes rendus de réunion, rapports, diagnostics antérieurs), observations (des pratiques, des événements).

Les données quantitatives sont utiles pour élaborer un diagnostic mais rendent rarement compte des évolutions et de la dimension historique de l'organisation. Pour cela, il faut reconstituer des éléments de son histoire à partir d'informations issues par exemple d'analyses biographiques de membres ou de responsables de l'organisation. Ce travail est souvent l'occasion de repérer les étapes marquantes dans l'histoire de l'organisation et les facteurs qui ont joué un rôle dans les dynamiques de renforcement, mais aussi de crise, de conflit voire d'affaiblissement de l'organisation. 
Lors des entretiens, on collecte souvent deux types d'information : des faits particuliers, qu'il convient de vérifier et croiser, et des analyses ou impressions livrées par les personnes enquêtées. Ces éléments, plus subjectifs, ont néanmoins une grande importance car ils reflètent l'atmosphère, les tensions, les perceptions qui conditionnent aussi le dynamisme d'une organisation (encadré 29). Lanalyse des documents archivés, pas toujours très nombreux, peut venir compléter les données d'entretien.

Prendre en compte la dynamique d'une organisation et son évolution dans le temps est souvent essentiel dans une démarche de diagnostic, parce que cela permet de comprendre certaines caractéristiques actuelles. Il s'agit également de soulever des questions qui mettent en lumière des éléments qu'on pourrait ignorer. Prenons par exemple le cas de l'évolution des effectifs des membres : si on note seulement «50 membres», ce n'est pas la même chose que de préciser «si cela résulte d'une scission d'une OP qui en comptait 150 », ou «si on est parti d'un groupe de $25 »$.

Par ailleurs, la prise en compte de la dynamique des organisations et de leur évolution dans le temps est souvent indispensable pour élaborer une typologie et chercher à répondre à une question précise. Identifier plusieurs trajectoires d'évolution d'organisations permet souvent de comprendre des questions qu'une organisation peut se poser.

\section{ID Répondre à une question spécifique}

Parfois, l'objectif recherché n'est pas un diagnostic global mais la réponse à une question bien ciblée. La démarche générale est la même que celle décrite précédemment : on identifie les variables et les critères pertinents en relation avec la question posée puis on cherche à renseigner ces critères. La question posée détermine les rubriques qui nécessitent d'être étudiées plus précisément. L'exemple présenté dans l'encadré 30 illustre le cas d'un diagnostic ciblé sur les services rendus par les organisations à leurs membres.

\section{Cas particulier du diagnostic d'une union ou d'une fédération}

Quand on s'intéresse à une union ou à une fédération, les critères mentionnés ci-dessus (historique, activités, organisation, relations avec l'extérieur) restent pertinents. On doit y ajouter cependant plusieurs 


\section{Encadré 29. Exemple d'outil : extrait d'une fiche de bilan «Cohésion entre les membres»}

L'organisme de recherche allemand GTZ a ainsi développé dans les années 1990 un outil de diagnostic fondé sur l'utilisation d'une série de 12 fiches bilan aidant à caractériser le fonctionnement et les capacités d'une OP. Ces fiches ont été produites en séminaires par les leaders d'OP concernées par l'auto-évaluation, et elles reflètent donc ce qui leur semble être de bons «indicateurs» (ou instruments). Les commentaires sont issus des discussions.

\begin{tabular}{|c|c|c|c|}
\hline Instruments & & Notation & Commentaires \\
\hline \multirow{4}{*}{$\begin{array}{l}\text { Cohésion : } \\
\text { finalités } \\
\text { communes }\end{array}$} & Les membres ont tous le même but & ++ & \multirow{4}{*}{$\begin{array}{l}\text { Est-ce que les } \\
\text { membres de } \\
\text { l'OP partagent } \\
\text { le même but? } \\
\text { Parfois ils ont pu } \\
\text { partager un but } \\
\text { en commun lors } \\
\text { de la fondation, } \\
\text { et l'ont perdu au } \\
\text { fil du temps }\end{array}$} \\
\hline & Discutent souvent, ce qui les tient ensemble & + & \\
\hline & $\begin{array}{l}\text { Au vu de l'argent ils oublient leurs buts du } \\
\text { début (la perspective d'un profit à court } \\
\text { terme peut faire oublier les objectifs de } \\
\text { départ, notamment la solidarité) }\end{array}$ & - & \\
\hline & $\begin{array}{l}\text { Plusieurs courants opposés se combattent au } \\
\text { sein de l'OP }\end{array}$ & -- & \\
\hline \multirow{4}{*}{$\begin{array}{l}\text { Respect } \\
\text { les uns } \\
\text { des autres }\end{array}$} & $\begin{array}{l}\text { On veille à ce que tous les groupes sociaux } \\
\text { soient au bureau du groupement }\end{array}$ & ++ & \multirow{4}{*}{$\begin{array}{l}\text { Beaucoup d'OP } \\
\text { s'arrêtent ou } \\
\text { stagnent parce } \\
\text { qu'un petit noyau } \\
\text { de membres } \\
\text { a accaparé le } \\
\text { pouvoir et que } \\
\text { les autres se } \\
\text { désintéressent }\end{array}$} \\
\hline & $\begin{array}{l}\text { Le bureau négocie ses décisions avec } \\
\text { les membres }\end{array}$ & + & \\
\hline & $\begin{array}{l}\text { On ne s'intéresse qu'à l'avis des plus } \\
\text { puissants }\end{array}$ & - & \\
\hline & $\begin{array}{l}\text { Chacun se méfie de l'autre et en dit } \\
\text { du mal }\end{array}$ & -- & \\
\hline \multirow[t]{5}{*}{$\begin{array}{l}\text { Qualité de } \\
\text { l'information }\end{array}$} & $\begin{array}{l}\text { En plus, on écrit les comptes rendus } \\
\text { et on les diffuse }\end{array}$ & ++ & \multirow{5}{*}{$\begin{array}{l}\text { Pour renforcer } \\
\text { la cohésion entre } \\
\text { les membres, } \\
\text { la permanence } \\
\text { et la qualité de } \\
\text { l'information, y } \\
\text { compris écrite, } \\
\text { sont des facteurs } \\
\text { importants }\end{array}$} \\
\hline & $\begin{array}{l}\text { À chaque réunion on présente } \\
\text { ce qui s'est passé et ce qui va se passer }\end{array}$ & + & \\
\hline & Tantôt on est informé, tantôt on ne l'est pas & $=$ & \\
\hline & Le bureau garde l'information pour lui seul & - & \\
\hline & $\begin{array}{l}\text { Tout circule sous forme de rumeur } \\
\text { vraie ou fausse }\end{array}$ & -- & \\
\hline
\end{tabular}

À noter que le choix des indicateurs et l'évaluation des modalités (notées $++,+,=,-,--)$ reposent sur une idée de «ce qui est souhaitable, ou positif», et nécessite aussi un débat qui constitue un moment important pour s'accorder sur une vision commune.

(d'après Lecomte et Pierret-Rieucaud, 1994) 


\section{Encadré 30. Étude de cas : le plan de l'enquête réalisée pour la Coordination nationale des organisations paysannes (CNOP) en Guinée}

Cette organisation faîtière, la CNOP, devait répondre à une demande du Programme d'Appui aux Communautés Villageoises pour préciser quel type d'appui permettrait à ses organisations membres de mieux satisfaire les besoins de leurs membres.

Le choix de l'échantillon a été raisonné pour représenter la diversité des OP dans les différentes régions en tenant compte de la production principale (les OP étant spécialisées par filière), du degré de structuration des OP et de leur composition.

Pour permettre un diagnostic sur les services rendus à leurs membres par les OP, un plan de l'enquête a été élaboré.

\begin{tabular}{ll}
\hline Caractéristiques de base de l'OP & $\begin{array}{l}\text { Contexte } \\
\text { Origine de l'organisation } \\
\text { Taille et composition } \\
\text { Types d'activités et de services rendus }\end{array}$ \\
\hline $\begin{array}{l}\text { Types de services offerts par l'OP } \\
\text { (par type de service) }\end{array}$ & $\begin{array}{l}\text { Description et analyse du fonctionnement } \\
\text { du service }\end{array}$ \\
& $\begin{array}{l}\text { Difficultés et contraintes rencontrées } \\
\text { dans le fonctionnement du service }\end{array}$ \\
& Solutions possibles envisageables \\
pour améliorer le service
\end{tabular}

(d'après Berthomé J. et. Pesche D., 2005. Mission d'appui à l'élaboration d'un modèle d'organisation des OP. Ministère du Plan, Programme d'Appui aux Communautés Villageoises. Conakry, Guinée, 52 p.)

points importants : la structure de l'organisation, la question de la subsidiarité et l'analyse de la diversité au sein de l'organisation.

La structure de l'organisation (voir également chapitre 3) est caractérisée par la nature des liens qui relient les différentes OP membres entre elles, les organes et unités de décision (organigramme) et les règles de désignation des représentants. Les textes qui régissent 
l'organisation sont la première source d'information mais, dans le cadre d'un diagnostic, il est nécessaire de confronter les textes à la réalité des pratiques, et de prendre en compte également la dimension personnelle des relations. C'est un des domaines où la prise en compte de l'histoire de l'OP est essentielle.

La question de la subsidiarité doit être approfondie quand il existe plusieurs niveaux au sein de l'organisation, par exemple l'organisation territoriale aux niveaux villageois, provincial, régional et national. Il importe alors de repérer quel niveau fait quoi et pourquoi, qui prend les décisions. Il faut également s'interroger sur l'efficacité des processus de prises de décision, ainsi que sur l'échelle pertinente pour résoudre les problèmes, mettre en œuvre des solutions ou gérer des ressources.

Enfin, la diversité doit être abordée sous deux angles : la description de cette diversité (qui sont les organisations membres?) et sa gestion (comment l'OP prend en compte les différentes catégories de membres qui peuvent avoir des attentes, des besoins, des moyens, des contraintes différents?). Un travail de typologie ou, au moins, de classification peut être utile dans les deux cas.

\section{Typologie d'OP}

Établir une classification ou une typologie d'OP est une variante de la démarche de diagnostic, appliquée à un ensemble d'OP, qui peuvent être reliées entre elles. Les informations recueillies et les critères retenus sont les mêmes que ceux que nous avons évoqués ci-dessus.

S'il faut faire la différence entre classification (descriptive) et typologie (explicative), dans tous les cas, il s'agit d'explorer et de rendre compte de la diversité. Dans quel but? Si on est intervenant extérieur cela permet de cibler les interventions, de faire d'éventuelles économies d'échelle. Si on fait partie d'un mouvement d'OP, c'est un outil pour comprendre ce que l'on est. Si on accompagne des OP, ce travail permet de tirer des enseignements sur ce qui marche en termes d'appui, sur ce qui est déterminant dans l'évolution d'une organisation. Pour les mêmes raisons, il peut constituer un excellent support à la réflexion dans un processus de renforcement de capacités des OP (voir encadré 31).

Une typologie n'est pas neutre, car les résultats et les conclusions dépendent fortement de l'angle d'observation et surtout de différenciation choisi. Par exemple, on peut décider que des OP sont proches car il s'agit de deux organisations de femmes maraîchères, 


\section{Encadré 31. Utilisation d'une typologie d'organisations de producteurs pour le renforcement de capacités}

Dans le cadre du projet pilote «Instruments et méthodes pour le renforcement des organisations », un voyage d'échanges a été organisé en 1998 pour un groupe de responsables de la FONGS (Fédération des ONG du Sénégal) et de la FUGN (Fédération Nationale des Groupements Naam au Burkina Faso). Les «paysans voyageurs» ont visité dix OP ivoiriennes. Lanalyse du fonctionnement de ces organisations en comparaison avec les OP burkinabè et sénégalaises a permis aux paysans de dégager quatre logiques qui polarisent le paysage des organisations de base : une logique professionnelle, une logique développement, une logique coutumière et une logique populaire. À chacune de ces logiques, correspondent des valeurs particulières et des modes de fonctionnement. En réfléchissant au positionnement de leur propre organisation par rapport à ces quatre logiques, les paysans ont été amenés à dégager des points forts et des faiblesses de leur propre OP.

(d'après Barbedette L., 1998. Une lecture de la diversité des modes d'organisation paysans. Regards paysans sur 10 OP au Burkina, en Côte d'Ivoire, en Guinée et au Mali. Document 34, projet pilote «Instruments et Méthodes pour le renforcement des organisations » Dpt. 403 GTZ/FUGN/FONGS 27 p.)

mais on peut aussi penser qu'elles sont très différentes car l'une est engagée dans un processus de développement accéléré avec recours à l'emprunt et un équipement sophistiqué, alors que l'autre ne gère collectivement que la commercialisation des productions, conduites de façon traditionnelle. Tout dépend de la question posée au départ : dans cet exemple, on peut se focaliser sur le type de membres et l'activité principale, ou bien sur la stratégie de développement adopté et la forme de production.

En conclusion, à condition de respecter quelques principes élémentaires, une démarche de diagnostic peut contribuer au renforcement des OP concernées de deux façons :

- par ses résultats qui dégagent les forces et les faiblesses des organisations et en donnent une explication. Les organisations ou leurs partenaires disposent ainsi de pistes pour l'action;

- par la démarche elle-même, qui contribue à renforcer les capacités des personnes impliquées en les amenant à s'interroger sur leurs pratiques, les résultats obtenus, les difficultés rencontrées. Cette dimension pédagogique du diagnostic suppose bien sûr d'adopter des outils participatifs. 


\section{$\sqrt{\text { Conclusion }}$ Pour des programmes complets et cohérents d'appui aux organisations de producteurs}

Il est généralement admis qu'un développement rural durable suppose une implication équilibrée de tous les acteurs concernés. C'est pourquoi de nombreux partenaires du développement estiment aujourd'hui important de soutenir les OP (voir chapitre 2). Il peut s'agir des États, des coopérations bilatérales, des institutions internationales d'aide au développement (Banque mondiale, FIDA), des agri-agences ${ }^{9}$, des nombreuses ONG. Dans la pratique, la volonté d'accompagner les OP est plus souvent portée par des acteurs extérieurs (ONG internationales, bailleurs de fonds) alors que les États et d'autres acteurs nationaux (ONG nationales, entreprises privées) peuvent avoir des réticences à voir le pouvoir des OP s'accroître réellement. Dans ces contextes instables, des programmes d'appui aux OP sont élaborés, soit sous forme de programmes spécifiques, soit comme composantes de programmes plus larges axés sur des thématiques variées (commercialisation et filières agricoles, gestion des ressources naturelles, décentralisation, sécurité alimentaire).

Dans le dernier chapitre, nous proposons des éléments qui peuvent servir à concevoir ou améliorer des programmes de large amplitude visant à renforcer les capacités d'un grand nombre d'OP dans la durée. Ce type de programme peut être élaboré par des acteurs extérieurs aux OP (États, bailleurs de fonds, ONG) mais aussi par des réseaux d'OP structurés aux échelles nationales et de plus en plus à l'échelle régionale, comme le ROPPA, EAFF, et qui se sont mis en place aux cours des dernières années.

\footnotetext{
${ }^{9}$ Les agri-agences sont des associations émanant des organisations professionnelles agricoles des pays du Nord, dédiées à la coopération internationale. Elles sont regroupées depuis 2003 dans AgriCord (www.agricord.org).
} 
Un élément fondamental, qui devrait être présent à chaque étape du raisonnement que nous allons suivre, est que les OP faittières ${ }^{10}$ soient associées aux nombreuses décisions nécessaires à la conception et la mise en route de programmes de renforcement des capacités des OP. Cela peut paraître une évidence mais cette recommandation pose rapidement la question de la légitimité et de la représentativité que l'État accorde à ces interlocuteurs paysans structurés de diverses manières. En effet, il n'existe pas toujours de structure unifiée de représentation nationale des producteurs. En Afrique de l'Ouest, cette structure existe presque partout dans la mesure où cela a été un objectif du ROPPA que de faciliter la mise en place d'une plateforme de représentation des OP dans chaque pays de la région. Cette question du «dialogue politique» entre l'État, ses interlocuteurs (les bailleurs de fonds) et les plateformes des OP est une question sensible, qui renvoie aux dynamiques politiques et sociales de chaque pays.

Les programmes d'appui aux OP ou de renforcement des capacités des OP ont généralement une durée de quelques années et peuvent être reconduits une ou plusieurs fois. La conception de ce type de programme suppose de s'interroger sur quelques grandes questions structurantes.

- Quelles OP veut-on appuyer et autour de quelles grandes fonctions? - Quels types d'actions mettre en œuvre? Quels moyens doivent être mobilisés, de quelles façons et par qui?

\section{Quelles OP veut-on appuyer et autour de quelles grandes fonctions?}

Quand un ministère ou un bailleur de fonds s'interroge pour concevoir un programme de renforcement des capacités des OP, il envisage généralement de travailler à l'échelle d'un pays tout entier ou au moins de quelques régions. Cela veut dire qu'il envisage d'intervenir dans un espace où existe déjà une grande diversité d'organisations, généralement regroupées aujourd'hui sous forme de fédérations, réseaux ou cadres de concertation.

Quand un réseau international d'OP souhaite apporter son soutien à ses membres, le plus souvent diversifiés, la question du choix des OP

\footnotetext{
${ }^{10}$ Cette expression permet de regrouper un ensemble de structures aux statuts divers (fédérations, cadres de concertation, syndicats agricoles) qui ont la particularité d'intervenir à l'échelle régionale mais le plus souvent national et qui représentent un nombre significatif d'OP.
} 
à aider n'est pas trop complexe puisqu'on suppose que le réseau appuiera principalement ses membres.

En revanche, pour des acteurs extérieurs au monde paysan (administrations, ONG, bailleurs de fonds), la question reste entière. L'expérience montre que les intervenants extérieurs articulent leur réflexion autour de trois axes :

- Faut-il privilégier une approche par filières ou plutôt territoriale?

- Faut-il privilégier une approche par les fonctions technico-économiques ou plutôt travailler sur la représentation syndicale?

- Faut-il faire des choix selon le degré de structuration, c'est-à-dire privilégier l'appui aux OP à la base, aux OP intermédiaires ou à celles qui existent à un échelon national?

Un travail de capitalisation de plus de dix ans d'expérience de la coopération française, conduit en 2004, montre qu'il convient justement de ne pas faire de choix trop tranchés à partir de ces questions, au risque de conduire des actions qui seraient de nature à fragiliser la construction institutionnelle des OP. Il est en général plus judicieux de privilégier la construction d'articulations entre des approches souvent complémentaires. En effet, ce sont ces articulations et ces synergies, qui, au-delà des spécialisations fonctionnelles de certaines organisations, contribuent à la construction de mouvements paysans efficaces et représentatifs.

Sur la question de l'échelon, il peut être judicieux de mettre l'accent sur les niveaux nationaux et intermédiaires des OP car l'appui des groupements de base peut être conçu comme une prérogative, au moins partielle, des unions et des fédérations existantes. Il est aussi important de centrer le travail autour de thèmes fédérateurs, d'enjeux partagés entre des OP de différentes natures. Il convient enfin de veiller à la complémentarité et à la cohérence de démarches et d'approches nécessairement plurielles et pragmatiques, dans des contextes changeants et fortement évolutifs : on peut citer par exemple l'appui à apporter aux OP en matière d'analyse et de construction de positions sur les questions foncières, sur le financement de l'agriculture, sur les services agricoles.

Les programmes de renforcement des capacités des OP ont du sens dans la mesure où ils contribuent à l'établissement de relations équilibrées entre les OP et leurs interlocuteurs que sont les organismes d'appui technique, le secteur privé et les pouvoirs publics. Dans chacun des cas, les enjeux et les difficultés diffèrent donc des capacités à renforcer également. 


\section{IID OP et services techniques}

Les relations entre les OP et les organismes d'appui technique prennent des formes différentes. Leur succès repose sur la capacité des OP à combiner :

- le renforcement des compétences de leurs ressources humaines internes (formation de responsables, échanges, recrutement de personnel technique);

- la mobilisation de compétences extérieures, soit ponctuellement (prestataires), soit de manière plus durable par la mise en place de dispositifs de services dont elles contrôlent les orientations.

On peut souligner l'importance d'anticiper la mise en place de nouveaux services en agissant en amont de leur montage et en veillant à l'implication des OP dans la conception des nouveaux projets. La question de la pérennité institutionnelle (financière et organisationnelle) de ces nouveaux dispositifs est essentielle.

Les réformes des services agricoles ont souvent conduit à la création de nouvelles agences spécialisées dans le conseil agricole dans lesquelles les OP jouent un rôle, souvent encore trop limité. C'est le cas par exemple de l'Agence nationale de conseil agricole et rural (ANCAR) au Sénégal où les OP siègent au conseil d'administration de la structure nationale mais sans toujours pouvoir réellement infléchir les orientations de l'institution. Plus localement, certains projets ont cherché à mettre sur pied des centres de services relativement autonomes dans lesquels les OP jouaient un rôle central.

\section{ID OP et acteurs économiques}

On peut remarquer tout d'abord que les OP sont, pour la plupart d'entre elles, des acteurs économiques dans la mesure où elles accompagnent la production et la mise en marché de produits agricoles ou d'élevage. Dans certaines zones et pour certains produits, les OP sont même devenues des acteurs majeurs du domaine économique ${ }^{11}$. Des programmes de renforcement interne des capacités des OP à remplir leurs fonctions économiques existent; ils portent sur des fonctions opérationnelles précises (gestion, stockage et logistique, mise en marché, négociation) souvent dans une filière bien identifiée.

\footnotetext{
${ }^{11}$ On peut citer les OP cotonnières d'Afrique de l'Ouest, mais également les organisations de producteurs de café dans certains pays d'Amérique latine.
} 
Il convient de noter que l'environnement institutionnel est souvent peu favorable à l'activité économique des OP. Dans ce domaine, des progrès sont à faire pour le rendre plus sécurisé et plus incitatif. Dans de nombreuses filières, les OP sont engagées dans des cadres de concertation avec les autres acteurs économiques concernés. Ces cadres peuvent prendre des formes juridiques spécifiques, comme les interprofessions, ou plus simplement des formes diverses de concertation. Un point central pour un programme de renforcement des capacités des OP est de travailler sur l'amélioration du fonctionnement de ces cadres de concertation de telle sorte qu'ils soient plus transparents et plus équitables. Cela passe par différentes actions de natures complémentaires :

- un élargissement des modalités de représentation contribuant à accroître le poids des producteurs;

- des appuis spécifiques aux collèges des producteurs qui abordent souvent les négociations en situation défavorable, notamment en termes de formation et d'information;

- et surtout la mise en place de mécanismes de financement adaptés aux initiatives des OP (fonds de roulement, crédits de commercialisation, etc.).

Il est aussi souvent utile de prévoir un accompagnement à la réflexion des OP pour guider leur choix face à des questions très diverses : par exemple, comment concilier la stimulation des initiatives locales et leur nécessaire autonomie et la concentration de certaines fonctions justifiées par la recherche d'économies d'échelle? Comment se positionner de façon juste vis-à-vis des autres acteurs et notamment évaluer l'avantage comparatif qui existe pour l'organisation à assumer tel ou tel rôle ou à le laisser jouer à d'autres acteurs spécialisés?

Dans certains cas, les producteurs participent au capital de sociétés de transformation ou de commercialisation (cas des filières coton notamment), un accompagnement de leur réflexion stratégique est alors utile à envisager.

D’une manière plus générale, la réflexion prospective et la capacité d'anticipation sont essentielles, en particulier quand il s'agit de faire des choix d'investissement, d'identifier des filières porteuses à moyen ou à long terme, de se positionner vis-à-vis de producteurs d'autres régions ou d'autres pays. Pour cela, la connaissance approfondie des enjeux liés aux filières concernées et aussi d'autres aspects stratégiques (mécanismes de financement, législation) constitue un atout déterminant et suppose souvent des complicités et des alliances avec des personnes ressources expérimentées. 


\section{OP et pouvoirs publics}

Dans la plupart des pays, que ce soit au niveau local ou national, les OP sont désormais généralement reconnues officiellement par les pouvoirs publics comme des interlocuteurs légitimes. Mais cette reconnaissance de principe peut se traduire de manière très différente dans les faits. Dans certains cas, les OP peuvent être réellement associées à la réflexion et aux décisions en matière de politique agricole, de développement rural, d'aménagement du territoire. Dans d'autres, elles sont formellement associées mais on ne leur donne pas réellement les moyens de jouer un rôle effectif (convocations tardives, information incomplète voire absente). L'enjeu est alors pour les OP d'obtenir la mise en place d'un cadre législatif et réglementaire qui entérine leur participation effective aux décisions qui les concernent. Mais cette reconnaissance, quand elle existe, est fragile et peut être remise en cause à tout moment, comme le montre l'expérience du CNCR au Sénégal, où cette organisation pourtant solide et expérimentée s'est vue exclue pendant plusieurs années par le Gouvernement de plusieurs cadres de discussion et de mécanismes de décision ou de mise en ouvre des politiques agricoles et rurales.

La multiplication des réunions, commissions, cadres de concertation, tant au niveau local, national, que sous-régional, ou même mondial représente un défi pour les OP qui ne disposent pas toujours des ressources humaines suffisantes en nombre et en qualité. D'où l'importance de la formation des responsables paysans et des techniciens des OP. La question des moyens financiers à mobiliser pour participer à ces réunions se pose également mais est peut-être plus facile à résoudre si le rôle des OP est reconnu (voir ci-dessous).

Enfin, lorsqu'elles sont engagées dans des processus politiques, les OP sont obligées de maintenir à jour une réflexion sur les grandes questions d'actualité afin de pouvoir se positionner utilement dans les débats et les négociations, par exemple sur la question des organismes génétiquement modifiés, sur les accords de partenariat économique (APE) (encadré 32).

Comprendre l'engagement d'une organisation ou d'un mouvement paysan dans un processus de négociation nécessite de bien comprendre la nature de ce processus, son déroulement dans l'espace et dans le temps. Trois caractéristiques principales peuvent être analysées à cet effet : l'étendue de la négociation dans l'espace (échelles géographiques et lieux de la négociation), l'étalement et le rythme de la négociation 


\section{Encadré 32. Les organisations de producteurs et la négociation des politiques : l'exemple des accords de partenariat économique et du foncier}

L'Initiative prospective agricole et rurale (IPAR) a réalisé, avec l'appui du CIRAD, un travail de capitalisation de l'expérience du rôle joué par les OP sénégalaises dans la négociation des politiques foncières de ce pays mais aussi par le ROPPA dans la négociation des accords de partenariat économique entre l'Europe et l'Afrique de l'Ouest. Ce travail met en relief l'importance de la prise en compte de la «forme» des négociations pour ajuster le travail de mobilisation et d'influence des OP en conséquence.

(temps et processus), et la nature de la thématique, plus ou moins proche des préoccupations directes des producteurs. Des ressources humaines, financières, politiques et sociales sont mises en ouvre pour influer sur les processus de négociation. La mobilisation de ces ressources diverses par les OP se fera de façon différente selon les caractéristiques et le stade de la négociation, par exemple au moment de l'entrée dans le processus ou aux étapes ultérieures. Laccompagnement des OP dans ces domaines prendra des formes différentes selon la durée et la nature des processus de négociation dans lesquels elles sont engagées.

Force est de constater que les partenaires qui affichent une volonté de renforcer les OP n'ont pas toujours œuvré concrètement dans ce sens. Ainsi, dans certains pays d'Afrique de l'Ouest et à Madagascar, la mise en place sans préparation suffisante des Chambres d'agriculture dont les attributions recoupent celles des OP existantes a pu perturber certaines dynamiques de structuration d'OP. De même, la création accélérée et imposée de plateformes nationales a parfois généré de la confusion et a remis en cause la légitimité de fédérations en construction. Les pouvoirs publics et leurs interlocuteurs, les bailleurs de fonds, ont donc une responsabilité majeure dans la construction de capacités dans la durée.

\section{Quelles actions mettre en œuvre? Quels moyens mobiliser et par qui ?}

Les programmes de renforcement des capacités des OP comprennent en général plusieurs domaines d'action qui concernent notamment les compétences, l'institutionnalisation et le financement. 
Le renforcement des compétences, proprement dites, comprenant des actions de formation, d'échanges d'expérience, de voyages d'étude, etc., ne produit généralement pas d'effets immédiats ni facilement visibles mais il est pourtant déterminant pour «le renforcement des OP dans leur capacité à jouer leur rôle» ou empowerment. Ces compétences peuvent concerner les organisations elles-mêmes mais également les acteurs de leur environnement. En effet, les limites que peuvent rencontrer les OP dans leur développement sont relatives au manque d'écoute et de connaissance de leurs interlocuteurs des ministères ou des organisations partenaires. Certaines actions de formation, d'échange d'expérience, de voyage d'étude gagnent à associer un public panaché entre représentants des OP et leurs interlocuteurs afin d'améliorer les conditions d'un dialogue par le partage d'expériences vécues et la construction d'analyses conjointes sur les enjeux et la façon de les aborder.

Les actions de renforcement de compétences participent aussi bien à l'amélioration des compétences techniques que stratégiques des OP : il s'agit de réflexion stratégique et prospective, de construction d'une vision sur l'avenir de l'agriculture et de sa place dans la société (voir chapitre 5).

Lexpérience du dispositif de formation des cadres du CNCR au Sénégal illustre bien ces différents aspects (voir encadré 33).

Le renforcement institutionnel vise à permettre à certaines organisations de renforcer leur pérennité en combinant à la fois un soutien financier pour la création et l'équipement d'un siège de l'organisation par exemple, et humain par le recrutement de salariés. Le défi pour certaines OP d'envergure régionale ou nationale est de disposer de ressources humaines internes suffisantes pour mener à bien leurs missions. Dans ce domaine, la construction de relations de confiance et de complémentarité entre salariés et responsables paysans est stratégique pour l'avenir d'une organisation. C'est une dimension qu'il est utile de prendre en compte au moment de concevoir des programmes.

Des mécanismes de financement innovants sont développés parallèlement aux actions précédentes. Les OP manquent en effet souvent de ressources financières pour remplir leurs missions, même si, selon leur nature, celles-ci peuvent éventuellement bénéficier de financements spécifiques.

Ainsi, les services économiques aux membres ont pour objectif de s'équilibrer au plan financier. Ceci est cependant rarement possible 


\section{Encadré 33. Former les responsables des organisations de producteurs de différents niveaux : exemple du Programme des services agricoles et appui aux organisations de producteurs (PSAOP II)}

Au Sénégal, une composante d'un programme soutenu par la Banque mondiale (Programme des services agricoles et appui aux organisations de Producteurs, PSAOP II) avait pour objectif de renforcer les capacités des OP aux niveaux local, régional et national, à défendre les intérêts des exploitations familiales et à faciliter à ces dernières l'accès à des services techniques et économiques. Cela permettrait d'accroître durablement les productions, la sécurité alimentaire et les revenus. Il s'agissait en particulier de renforcer d'abord la représentativité et l'inclusion sociale des organisations aux niveaux local et régional, ensuite, la participation des OP au dialogue sur les politiques agricoles et rurales et, enfin, la maîtrise technique et économique des activités par les exploitations familiales et leurs organisations. Lobjectif était aussi de renforcer les capacités stratégiques des responsables pour leur permettre d'influer efficacement sur l'élaboration des politiques agricoles et rurales et de participer activement à la mise en œuvre des programmes de développement.

Pour atteindre cet objectif, trois activités ont été réalisées :

- la formation des responsables professionnels aux niveaux national, régional et local;

- la circulation de l'information et la communication au sein du mouvement professionnel agricole;

- la concertation d'une part entre $\mathrm{OP}$ et, d'autre part, entre ces dernières et les autres acteurs du développement agricole et rural.

La Fédération des ONG du Sénégal (FONGS) a été retenue pour mettre en œuvre le programme de formation des responsables d'organisations de producteurs au bénéfice de l'ensemble des OP du Sénégal. Au total, entre 2008 et 2011, près de 300 leaders paysans ont été formés dans les différentes régions du pays ainsi que 40 au niveau national. Ces formations ont permis aux responsables d'acquérir une meilleure compréhension du contexte international, des enjeux nationaux de développement agricole et rural, des contraintes à l'amélioration des performances des filières et de l'environnement socio-économique des exploitations familiales. Indirectement elles ont également contribué à faire émerger de jeunes responsables au niveau local. Enfin, elles ont constitué une occasion pour l'organisation de mener une réflexion sur son fonctionnement interne et sur ses relations avec l'extérieur. 
en intégralité, surtout pour des OP jeunes. Ce type d'activités peut être financé par les canaux classiques du financement de l'agriculture (crédit, micro-finance) dans des conditions à négocier. En outre, souvent, des soutiens sous forme de subvention ou de fonds de garantie sont utiles pour amorcer l'activité. Le financement du fonctionnement de l'organisation (salariés, siège, organisation des réunions) devrait être principalement couvert par les ressources propres (cotisations et revenus d'activités) mais l'est rarement totalement. Enfin, pour les missions d'intérêt général telles que la participation à l'élaboration des politiques agricoles, mais aussi l'alphabétisation, ou la formation au métier d'agriculteur, si elles ne sont pas ou mal assumées par les États, il n'est pas juste de prétendre les faire financer par les ressources propres des OP. Pour cette raison, des financements publics sont justifiés, en veillant à ce que les modalités de mobilisation et de mise à disposition de ces fonds n'entraînent pas une dépendance accrue de l'organisation à l'égard des pouvoirs publics.

Les moyens financiers mobilisés dans les programmes d'appuis aux OP (ou les composantes d'appui aux OP au sein de programmes plus globaux) sont généralement des fonds souples ${ }^{12}$, qui permettent de financer par des mécanismes de fonds compétitifs ou autres, des projets des organisations, y compris les actions de renforcement de capacités évoquées ci-dessus. Cela a été le cas par exemple des projets PNDSA (Programme national de développement des services agricoles) financés par la Banque mondiale dans plusieurs pays, notamment le Sénégal et le Burkina Faso, mais également des programmes d'appui à la professionnalisation de l'agriculture de la coopération française (Mercoiret et al., 2004).

Lenjeu central pour ces programmes d'appui est de mettre en place des mécanismes de financement pérenne du fonctionnement des OP, qui ne repose pas uniquement sur des programmes et projets, par nature éphémères. Dans ce sens, les deux possibilités principales généralement envisagées sont la mise en place de fonds dédiés et alimentés par des taxes sur les produits ou le commerce agricoles ou des appuis budgétaires votés par le Parlement. La mobilisation de fonds publics pour le soutien aux OP se justifie par le fait qu'elles remplissent certaines fonctions d'intérêt général (voir chapitre 2). S'agissant de fonds publics la contrepartie est l'obligation de rendre des comptes sur leur emploi. Dans tous les cas, il est souhaitable pour les OP de garder un volant de ressources propres qui assure leur indépendance.

12 C'est-à-dire des fonds dont l'imputation budgétaire reste ouverte pour permettre de s'adapter aux besoins : appui institutionnel, formation, activités productives. 


\section{ID Comment canaliser les moyens pour le renforcement des capacités des OP?}

Jusqu'à maintenant, il est assez rare que les OP elles-mêmes aient été associées pleinement à la conception des programmes mis en place pour les renforcer. Ceux-ci portent la marque de leurs initiateurs, bailleurs de fonds ou opérateurs de développement qui peuvent avoir des visions différentes sur ce qu'il convient d'apporter comme appui aux OP.

La Banque mondiale, par exemple, a privilégié la constitution de fonds compétitifs pour donner aux OP locales des moyens pour prendre en charge certaines activités prédéfinies, sans toujours prendre en compte, sauf au Sénégal, les fédérations paysannes dont ces OP locales étaient membres. Dans quelques pays où les OP faîtières sont suffisamment solides, elles ont pu négocier la gestion partielle des fonds alloués au renforcement des OP; cette position de responsabilité est très utile pour renforcer les capacités, comme cela a été le cas du Sénégal.

Pendant plus de dix ans, plusieurs programmes soutenus par la coopération française ont facilité l'émergence d'OP faîtières et l'organisation d'un dialogue entre les OP et l'État, notamment en Afrique de l'Ouest. Des programmes dits de "professionnalisation de l'agriculture» ont permis de renforcer certaines OP qui aujourd'hui constituent les piliers solides de plateformes paysannes nationales. Ce type de programme a été associé à une assistance technique proche des ministères de l'agriculture, mais suffisamment indépendante pour faciliter ces rapprochements en mobilisant des moyens financiers en définitive assez limités.

Depuis les années 1990, les agri-agences de plusieurs pays (France, Pays-Bas, Canada), regroupées dans AgriCord, multiplient les formes de partenariat direct avec une ou plusieurs OP dans les pays du Sud. Ces relations directes combinent des appuis classiques à des formes de partenariat de type plus syndical où des organisations professionnelles échangent leurs visions, analyses et modes d'action en tant qu'organisations représentant les producteurs.

Les États peuvent jouer également un rôle important dans le renforcement de capacités des OP mais aussi et surtout des agriculteurs et ruraux par la formation agricole initiale et la formation professionnelle. Ce travail de fond est indispensable mais produit des effets sur le long terme. L'État joue aussi un rôle clé en fixant le contexte législatif 
et réglementaire, qui sera favorable s'il implique la liberté d'association, l'existence de statuts types, des interlocuteurs bien identifiés pour les OP. C'est pourquoi, dans certains cas, les dispositifs d'appui aux OP ont également inclus des actions de sensibilisation et de renforcement de capacités des agents des ministères concernés.

\section{Perspectives}

À l'avenir, les programmes d'appui devraient impérativement prendre en compte la structuration croissante des OP et négocier avec elles les objectifs, contenus et modalités des programmes dont elles sont parties prenantes et bénéficiaires. Cette posture permettrait d'une part de renforcer les OP existantes et non de les affaiblir, d'autre part aux organismes désireux de les appuyer, de s'assurer la possibilité d'une meilleure adéquation des dispositifs proposés aux besoins des OP. Dans ces programmes, il s'agirait de créer les conditions de la pérennité, ce qui nécessite un partenariat conçu comme une relation dans la durée, qui explicite les objectifs et les intérêts respectifs des différentes parties, les exigences réciproques, qui prévoit la négociation régulière des objectifs, des activités et des modalités du suivi-évaluation, une capacité réciproque d'adaptation favorisée par une communication réelle entre les parties, etc. Des outils adaptés sont indispensables à un partenariat efficace, ainsi les conventions de coopération devraient inclure l'engagement des OP, les comités de pilotage devraient être de taille limitée pour être efficaces, l'affectation des financements devrait être souple, etc. Les fonds compétitifs peuvent être critiqués dès lors qu'ils risquent de marginaliser les organisations les plus faibles, il apparaît alors important de prévoir des mécanismes pour réduire cette asymétrie entre organisations.

Dans un contexte de crises économiques, face au risque de réduction de l'aide au développement, et des programmes de renforcement de capacité des OP, l'enjeu pour les OP est de négocier l'institutionnalisation de dispositifs d'appui, notamment dans deux domaines essentiels.

Le premier domaine est celui de la formation des leaders. Cette tâche n'est en effet jamais terminée, car il est nécessaire pour la vie démocratique des organisations de renouveler leurs cadres, ce qui suppose l'existence d'un vivier de (jeunes) responsables. De plus, nous avons vu tout au long de ce manuel que la capacité des organisations à répondre aux besoins de leurs membres aussi bien qu'aux attentes de leurs partenaires dépend largement des compétences en leur sein. La mise en 
place de dispositifs de formation pérennes est dès lors un point capital, à préparer dans le cadre des programmes encore existants, ou à négocier avec les pouvoirs publics ou avec les partenaires institutionnels quand les programmes sont clos.

Le second est celui des services aux producteurs et aux organisations de base. Il s'agit d'accompagner la création de dispositifs de services autonomes par rapport aux logiques de projets et de programmes. En effet, beaucoup de programmes de renforcement soutiennent des actions intéressantes mais qui périclitent dès que les appuis financiers externes ne sont plus là. La prise en charge d'une partie des coûts des services techniques par les usagers tend à se généraliser. Il convient donc de soutenir les bénéficiaires de services dans la recherche des ressources nécessaires par la mise en place d'activités génératrices de revenus et la construction de partenariats complémentaires. Cependant, comme nous l'avons vu, il est peu réaliste de miser uniquement sur ces ressources pour pérenniser les services. Lautonomie peut alors reposer sur la combinaison de plusieurs partenariats ou de projets s'étalant sur des périodes différentes, en complément des ressources propres. Il paraît aussi indispensable de penser des mécanismes de financement fondés sur une fiscalité propre (taxes professionnelles par exemple) ou sur la fiscalité générale de l'État afin d'assurer des ressources régulières, au moins partielles, à ces dispositifs.

Quoiqu'il en soit, la mise en place de dispositifs d'appui pérennes aux producteurs et aux OP, dans différents domaines (conseil agricole, information et communication, formation), est essentielle au renforcement durable des capacités nécessaire au développement agricole. De nombreux partenaires du développement sont désormais convaincus que les OP doivent jouer un rôle central dans la conception, la mise en place et la gestion de ces dispositifs. Le travail de plaidoyer en ce sens doit cependant se poursuivre. Cela suppose que dans toutes les organisations, les responsables actuels en soient convaincus et en trouvent le temps et les moyens. Nous espérons que cet ouvrage contribuera à cette prise de conscience et aidera à la construction des argumentaires. 



\section{Bibliographie}

Acheroy C., Hadjaj-Castro H., 2006. Fiche 3. Méthode de planification de projet par objectif (PIPO, PPO, PPPO, ZOPP). COTA, Bruxelles.

(http://www.cota.be/SPIP/IMG/pdf/ Fiche3_GCP_PIPO-2.pdf)

Agoua F., Mercoiret M.R., Ouikoun M., 2000. Le renforcement des organisations paysannes du Zou (Bénin). CIRAD, Montpellier. $150 \mathrm{p}$.

Banque mondiale, 2006. Rapport annuel 2006. The World Bank, Washington, D.C.

http://siteresources.worldbank.org/INTANNREP2K6/

Resources/2838485-1158549322105/

WBAR06French.pdf

Banque mondiale, 2007. Rapport sur le développement dans le monde 2008. L'agriculture au service du développement. The World Bank, Washington D.C.

Benkahla A., Faye J., Touré O., Seck S.M., Ba C.O., 2011. Les organisations paysannes sénégalaises dans le processus de réforme foncière. Initiative Prospective Agricole et Rurale, Dakar 69 p.

Bennegouch N., Hassane M., 2010. Mooriben : l'expérience d'un système de services intégrés au bénéfice des paysans nigériens. Dynamiques paysannes, $23,8 \mathrm{p}$.

Bergeret P., 2003. Paysans, État et marchés au Vietnam. Dix ans de coopération agricole dans le bassin du Fleuve Rouge. Karthala, Paris. 296 p.
Bourgeois R., Jesus F., Roesch M., Soeprapto N., Renggana M., 2003. Indonesia: Empowering Rural Producers Organization. Rapport pour la Banque mondiale, World Bank Contract, $\mathrm{n}^{\circ}$ 7122674. $108 \mathrm{p}$.

Brock K., McGee R., 2004. Mapping trade policy: understanding the challenges of civil society participation. Institute of development Studies, Brighton, Working Paper n 225 .

Celis Callejas F., 2000. Nuevas formas de asociacionismo en la cafeticultura mexicana: el caso de la CNOC. Mecanoescrito, s/p. Xalapa, Ver.

Diaz J., Le Coq J.F., Mercoiret M.R., Pesche D., 2004. Le renforcement des capacités des organisations paysannes et rurales, enseignements de l'expérience de la Banque mondiale. Banque mondiale, CIRAD-Tera, Montpellier. $40 \mathrm{p}$.

Draperi J.F., 2003. La coopération face aux nouvelles attentes de la société. In Les coopératives entre territoires et mondialisation, J.M. Touzard et J.F. Draperi, Cahiers de l'économie sociale $\mathrm{n}^{\circ}$ 2. L'Harmattan, Paris, $391 \mathrm{p}$.

Gentil D., Mercoiret M.R., 1991. Y a-t-il un mouvement paysan en Afrique noire? Tiers-Monde, 32 (28), 867-886.

Hadjaj-Castro H., Wilbeaux N., 2007. Fiche 6. Indicateurs de développement. COTA, Bruxelles. 11 p.

De Janvry A., Sadoulet E., 2004. Organisations paysannes et développement rural au Sénégal. Université de Californie, Berkeley, Banque mondiale, Washington, $97 \mathrm{p}$. et sur le site http://www.inter-reseaux.org/IMG/ article_PDF/article_a2079.pdf 
Lecomte B., Pierret-Rieucaud M.H., 1994. Outils de diagnostic organisationnel des organisations populaires et des ONG en Afrique de l'Ouest et centrale, GTZ. Veröffentlichungen der Abtl. 403(19), Eschborn, Allemagne.

Léonard H., 2008. La Fédération des associations paysannes de Louga (Sénégal). Dynamiques paysannes, 18 , numéro spécial.

Mercoiret M.R., 1994. L'appui aux producteurs ruraux. Guide à l'usage des agents de développement et des responsables de groupements. Karthala, Paris. $463 \mathrm{p}$.

Mercoiret, M.R., Berthomé J., Pesche D., 2004. Principaux résultats du processus d'analyse et de capitalisation des programmes d'appui institutionnel aux organisations paysannes. Rapport de synthèse. Paris, ministère français des Affaires étrangères, CIRAD. 40 p.

Mercoiret M.-R., Pesche D., Bosc P.M., 2007. Les organisations paysannes et rurales pour un développement durable en faveur des pauvres. Contribution à la rédaction du Rapport sur le développement dans le monde 2008. L'agriculture pour le développement. Compte-rendu de l'atelier de Paris, 30-31 octobre 2006. Ministère français des Affaires étrangères, ministère de l'Agriculture, Banque mondiale, FIDA, CIRAD, Paris, versions française, anglaise et espagnole. $45 \mathrm{p}$.

Mercoiret M.R., Minla Mfou'ou J., 2006. OPR, renforcement du pouvoir des agricullteurs et action collective. Note introductive. In : Les organisations paysannes et rurales pour un développement durable en faveur des pauvres. Contribution à la rédaction du Rapport sur le développement dans le monde 2008. L'agriculture pour le développement. Compte rendu de l'atelier de Paris (30-31 octobre 2006). Ministère français des Affaires étrangères, ministère de l'Agriculture, Banque mondiale, FIDA, CIRAD, Paris. 14 p.

Mintzberg H, 1982. Structure et dynamique des organisations. Éditions d'Organisation, Paris (traduction française).

Mintzberg H, 1989. Le management. Voyage au centre des organisations. Éditions d'Organisation, Paris (traduction française).

Newell P., Tussie D. (eds), 2006. Civil Society Participation in Trade Policymaking in Latin America: Reflections and Lessons. Insitute of Development Studies, Brighton, grande-Bretagne, Working paper $\mathrm{n}^{\circ} 267,88 \mathrm{p}$.

Pesche D., 2001. Classification et typologies des organisations paysannes. AGRIDOC, Inter-Réseaux, 3 p.

Santana R., 1997. Les Indiens d'Équateur, citoyens dans l'ethnicité? CNRS, Centre régional des publications, Toulouse, France.

\section{Sites internet}

(consultations le 31/03/2012)

http://www.ipar.sn/spip.php?article283

http://www.agricord.org/

http://www.fongs.sn

http://www.ipar.sn

http://fapal.worldpress.com 


\section{Liste des sigles}

AFD : Agence française de développement

ALCA : Área de Livre Comércio das Américas - zone de libre-échange des Amériques

ANCAR : Agence nationale de conseil agricole et rural, Sénégal

APE : Accords de partenariat économique

APM : Agriculture paysanne et modernisation, Cameroun

APROCA : Association des producteurs de coton africains

CEDEAO : Commission de la Communauté économique des États d'Afrique de l'Ouest

CIEPAC : Centre international pour l'éducation permanente et l'action concertée, France

CIFA : Centre Interprofessionnel pour la formation aux métiers de l'agriculture, Sénégal

CIRAD : Centre de coopération internationale en recherche agronomique pour le développement, France

CNCR : Conseil national de concertation et de coopération des ruraux, Sénégal

CNOC : Coordinadora Nacional de Organizaciones Cefetaleras - coordination nationale des organisations productrices de café, Mexique

CNOP : Coordination nationale des organisations paysannes, Guinée

COPAC : Comité des organisations professionnelles agricoles de l'Union européenne
COPROFAM : Coordinadora de las Organizaciones de Productores del Mercosur

DFID : department of international development, Grande-Bretagne

EAFF : Eastern Africa Farmers Federation - Fédération des agriculteurs d'Afrique de l'Est

FAO : Food and Agriculture Organization of the United Nations Organisation des Nations Unies pour l'alimentation et l'agriculture

FENOCIN : Confederación Nacional de Organizaciones Campesinas, Indígenas y Negras, Équateur

FIDA : International Fund for Agricultural Development

FIPA : Fédération internationale des producteurs agricoles

FONGS : Fédération des ONG du Sénégal

FPA : Farmer's Professional Associations

FPFD : Fédération des Paysans du Fouta Djallon, Guinée

FUGN : Fédération nationale des groupements Naam, Burkina Faso

FUGPN : Fédération des Unions de groupements paysans du Niger

FUPRO : Fédération des Unions des producteurs, Bénin

GPC : Groupements de producteurs de coton, Afrique de l'Est

GTZ : Gesellschaft für Technische Zusammenarbeit - Coopération technique allemande, Allemagne

IPAR : Initiative prospective agricole et rurale

IRC : Institut des régions chaudes, France 
KENFAP : Kenya National Federation of Agricultural Producers KEPSA : Kenya Private Sector Alliance

MAE : ministère français des Affaires étrangères

MAP : ministère français de l'Agriculture et de la pêche

Mercosur : Mercado Común del Sur, Amérique du Sud

NEPAD : New Partnership for Africa's Development - Nouveau Partenariat pour le Développement de l'Afrique

OCDE : Organisation de coopération et de développement économiques

PAFFO : Pan African Farmers Forum

PROPAC : Plateforme régionale des organisations paysannes d'Afrique centrale
PSAOP : Programme des services agricoles et organisations de producteurs

ROPPA : Réseau des organisations paysannes et professionnelles d'Afrique de l'Ouest

SACAU : Southern African Confederation of Agricultural Unions

SADEC : Southern African
Development Community UEMOA : Union économique et monétaire ouest-africaine

UNPCB : Union nationale des producteurs de coton du Burkina Faso

UPAFA : Université paysanne africaine

USAID : United States Agency for International Development, États-Unis 


\section{Index}

accès au marché 91, 93

action collective 9, 20, 27, 33, 90

appartenance $45,49,65,71$

approvisionnement 16, 19, 25, 27, 91, 94

appui-conseil 70, 98

assemblée générale 41, 63, 67

auto-évaluation 56, 115, 121

autonomie 43, 64, 77, 86, 105, 107,113, 129, 137

biens partagés 99, 100

biens publics 10, 11, 98, 99, 100

bureau 41,63

capacités organisationnelles 32

capacités stratégiques $31,32,81,83,133$

capacités techniques 31,32

capitalisation $55,58,78,127$

chaîne de valeur 95

classification $115,116,123$

commercialisation 74, 90, 91, 94, 95, 129

commissaire aux comptes 67

communication 30, 40-41, 55, 71, 94, 136

compétitivité 93, 97

comptabilité 41, 65, 66

comptable 66, 69

confédération 22

conseil d'administration $63,64,67$

contrat 90, 94, 101

coopérative 10,53

crédit $27,70,95,96,97$

défense des intérêts 9, 104, 107

développement local 99, 100

diagnostic 48, 115-124

diversification 43, 108

diversité 12, 17, 23, 116, 122, 123, 124, 126

élus $48,55,63,66-71,74,80,81,115$

épargne 97

équipements collectifs 10

équité $30,44,53$
État 15, 17, 35, 98, 100, 106-108, 126, 135, 137

évaluation 52, 55, 56, 58, 117, 119, 121

exclusion 34,74

externaliser 67

faîtière 108,126

fédération 42, 43, 72, 120

filière $19,20,64,90,91,93,95,108,122,128$

financement 53, 64, 101, 107, 108, 113, 127, $129,131,132,134$

financement de l'agriculture 94, 101, 127, 134 foncier 107,131

formation $32,41,48,77,79-86,98,116,128-137$

gestion de l'information 71, 72, 94

gestion financière $65,66,80$

gouvernance $35,43,105$

groupement 17, 19

identité $32,39,45,80,118$

image $39,65,71,72,74$

inclusion 30,74

indicateur 57

information $19,32,55,71,72,79,90,91,93$, 94, 97, 101, 109, 112, 116, 117, 129, 130, 133 ingénierie de formation 80,82

innovation 71, 89, 97, 99

leader 74

légitimité 34, 74, 77, 100, 108, 117, 126, 131

maîtrise d'ouvrage 31

management $32,47,55,56$

manuel de procédure 62

membre 63,108

mise en marché $25,101,128$

mouvement paysan 21,22

négociation 29, 93, 110,113, 128, 130, 131, 136

objectif $34,52,74,115,116,126,132$

ONG 10, 19, 20, 89, 107, 108, 125, 127

organes $39,41,63,122$

organisations de producteurs $9,15,26,30,39$,

$77,103,115,124,125,127,128,131,133$

organisations paysannes $8,9,18,22,108,111$, 122 
organisations professionnelles agricoles 9,125 , 141

orientation 49, 106, 107

partage des rôles 69

partenaire 79,115

partenariat $89,91,94,97,98,100,101,111$, 130,135

plan d'action $47,49,52,53,55,80,82$

plan d'affaires 53

planification $47-55$

plan stratégique $47,48,50,52,55,58,115$

plateforme 42, 111, 126

politiques publiques $34,35,103,104,105,107$, $109,111,112$

politiques sectorielles 12,104

pouvoir de négociation 17, 33, 90, 91, 93

prestation $41,84,85,101$

prise de décision 42, 59, 62-65

programmes d'appui 19, 20, 125, 126, 134, 136

réflexion prospective 129

réflexion stratégique $32,44,47-50,52,55,56$, $80,82,129,132$

règlement intérieur $62,63,71$

relations internes 39

renforcement de capacités $29,77,80,85,123$, 134, 135

renforcement institutionnel 26, 27, 79, 132

représentation $9,28,35,39,63,101,104,106$, $107,108,113,126,127,129$ représentation des intérêts $35,79,104,106$, 107, 108, 113

représentativité 74, 126, 133

réseau $42,43,111,126$

responsable 67,119

ressources financières 64,132

ressources humaines $32,67,70,73,75,98,128$, $130,131,132$

ressources naturelles $11,17,99-101$

réunion $55,56,66,72,73$

salariés 41, 42, 44, 63-71, 84, 98, 132, 134

SEPO 57,58

services $11,16,25,27,32,33,41,70,79,89,91$, $98,99,118,120,122,132,134,137$

société civile 35, 100, 105, 107, 109, 110, 111

spécialisation $34,43,116$

statut $10,19,42,61,63,64,68$

statuts $10,18,45,61,63,64,67,71,119,126$

stratégie 41, 51, 61, 95, 104, 108

structuration 20, 22, 29, 42, 44, 122, 127, 131

structure 39, 41-44, 63, 74, 122

subsidiarité 43,123

suivi-évaluation 55-58, 136

trajectoire d'évolution 42

trésorier 69

typologie $115,116,118,120,123,124$

union $42,43,120$

valeurs $42,44,45,68,101,124$

vision $32,51,53,59,63,115,117,132$

vulgarisation $97,98,99$

Photo de couverture : Réunion d'un groupement de paysannes à Madagascar. (OCirad/ É. Penot

Édition : Claire Parmentier, Presses agronomiques de Gembloux Mise en pages : Hélène Bonnet

Impression : La Source d'Or

Dépôt légal : juillet 2012 
L'appui aux organisations de producteurs est un enjeu majeur de l'accompagnement des transformations de l'agriculture dans les pays du Sud. C'est un élément essentiel des politiques en faveur de l'agriculture soutenues par la Banque mondiale. Ainsi, beaucoup de gouvernements souhaitent aujourd'hui disposer d'interlocuteurs crédibles pour concevoir et mettre en œuvre des politiques agricoles et rurales efficaces. Malgré ce contexte favorable, les organisations rencontrent des difficultés pour accéder à l'information et aux ressources et pour les gérer au service de leurs membres. Cet ouvrage a pour objectif d'aider le lecteur à se poser « les bonnes questions » : pourquoi appuyer les organisations ? Comment renforcer l'organisation elle-même ? Comment améliorer les relations de partenariat avec les acteurs publics et privés? Illustrée d'exemples des pays du Sud, cette synthèse valorise des sources documentaires et l'expérience des formations dispensées par l'IRC à Montpellier dans le cadre du mastère "Acteurs du développement rural », animé par des chercheurs du CIRAD et du CIEPAC.

Cet ouvrage didactique est destiné aux acteurs du développement agricole de tous les pays.

Marie-Jo Dugué, agronome, se consacre au renforcement de capacités dans le monde rural en France et en Afrique. De nombreuses missions d'appui aux organisations en Afrique et en Asie complètent son expérience.

Denis Pesche, sociologue au CIRAD et spécialiste des organisations de producteurs, a animé des réseaux d'information et d'échange.

Il conduit des expertises sur le renforcement des organisations en Afrique et en Amérique centrale.

Jean-François Le Coq, agro-économiste au CIRAD, axe ses recherches sur les dynamiques agraires et rurales, les organisations de producteurs, les filières et les politiques publiques, les processus de renforcement de capacités des acteurs.
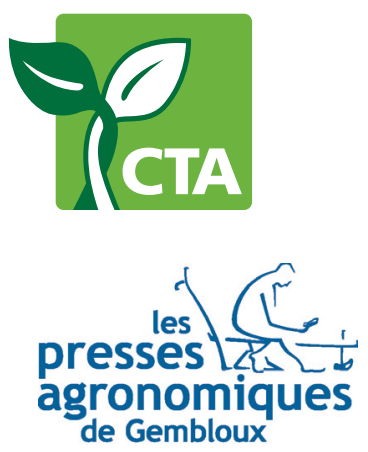

$18,30 €$

ISBN : 978-2-7592-1805-9

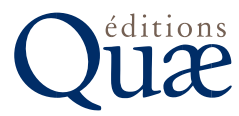

Éditions Cemagref, Cirad, Ifremer, Inra www.quae.com

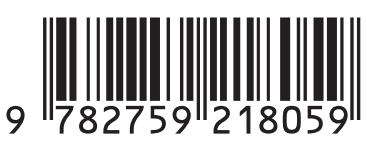

Réf. : 02324

ISSN : 1778-6568 